\title{
Integrated Data and Energy Communication Network: A Comprehensive Survey
}

\author{
Jie Hu, Member, IEEE, Kun Yang, Senior Member, IEEE, Guangjun Wen, Senior Member, IEEE, Lajos Hanzo, \\ Fellow, IEEE
}

\begin{abstract}
In order to satisfy the power thirsty of communication devices in the imminent $5 \mathrm{G}$ era, wireless charging techniques have attracted much attention both from the academic and industrial communities. Although the inductive coupling and magnetic resonance based charging techniques are indeed capable of supplying energy in a wireless manner, they tend to restrict the freedom of movement. By contrast, RF signals are capable of supplying energy over distances, which are gradually inclining closer to our ultimate goal - charging anytime and anywhere. Furthermore, transmitters capable of emitting RF signals have been widely deployed, such as TV towers, cellular base stations and Wi-Fi access points. This communication infrastructure may indeed be employed also for wireless energy transfer (WET). Therefore, no extra investment in dedicated WET infrastructure is required. However, allowing RF signal based WET may impair the wireless information transfer (WIT) operating in the same spectrum. Hence, it is crucial to coordinate and balance WET and WIT for simultaneous wireless information and power transfer (SWIPT), which evolves to Integrated Data and Energy communication Networks (IDENs). To this end, a ubiquitous IDEN architecture is introduced by summarising its natural heterogeneity and by synthesising a diverse range of integrated WET and WIT scenarios. Then the inherent relationship between WET and WIT is revealed from an information theoretical perspective, which is followed by the critical appraisal of the hardware enabling techniques extracting energy from RF signals. Furthermore, the transceiver design, resource allocation and user scheduling as well as networking aspects are elaborated on. In a nutshell, this treatise can be used as a handbook for researchers and engineers, who are interested in enriching their knowledge base of IDENs and in putting this vision into practice.
\end{abstract}

Index Terms-RF signals, wireless energy transfer (WET), wireless information transfer (WIT), simultaneous wireless information and power transfer (SWIPT), wireless powered communication networks (WPCNs), integrated data and energy communication networks (IDENs).

Jie Hu and Guangjun Wen are with the School of Information and Communication Engineering, University of Electronic Science and Technology of China, Chengdu, 611731, China, email: \{hujie, gjwen\}@uestc.edu.cn.

Kun Yang is both with the School of Computer Science and Electronic Engineering, University of Essex, Essex, CO4 3SQ, UK, and with the School of Information and Communication Engineering, University of Electronic Science and Technology of China, Chengdu, 611731, China, email: kunyang@uestc.edu.cn.

Lajos Hanzo is with the School of Electronics and Computer Science, University of Southampton, Southampton, SO17 1BJ, United Kingdom, email: lh@ecs.soton.ac.uk.

Jie Hu would like to gratefully thank the financial support of National Natural Science Foundation of China (NSFC), Grant No. 61601097, U1705263 and 61620106011 as well as that of the Fundamental Research Funds for the Central Universities, No. ZYGX2016Z011. This work is also sponsored by Huawei Innovation Research Program (HIRP) and by 111 project (No. B14039).

Lajos Hanzo would like to gratefully acknowledge the financial support of the EPSRC projects EP/Noo4558/1, EP/PO34284/1 as well as of the European Research Council's Advanced Fellow Grant QuantCom.

\section{NOMENCLATURE}

AC Alternative Current

ACO Alternative Convex Optimisation

AMI American Megatrends Incorporated

AP Access Point

ASK Amplitude-Shift-Keying

AWGN Additive White Gaussian Noise

BPSK Binary-Phase-Shift-Keying

BS Base Station

CDF Cumulative Distribution Function

CICO-MC Continuous-Input-Continuous-Output MemorylessChannel

CMOS Complementary Metal Oxide Semiconductor

CoMP Coordinated Multiple Point

CPL Constant Power Load

C-RAN Cloud Radio Access Network

CRN Cognitive Radio Network

CS Current Splitting

CSI Channel State Information

CVL Constant Voltage Load

DC Direct Current

DIDO-MC Discrete-Input-Discrete-Output MemorylessChannel

D2D Device-to-Device

FET Field Effect Transistor

GaAs Gallium Arsenide

GaN Gallium Nitride

HBT Heterojunction Bipolar Transistor

IDEN Integrated Data and Energy communication Network

IFT-DPA Inverse F-Type Doherty Power Amplifier

InGaP Indium Gallium Phosphide

IoT Internet of Things

LPF Low Pass Filter

MAC Medium Access Control

MIMO Multiple-Input-Multiple-Output

MISO Multiple-Input-Single-Output

mmW millimetre Wave

NASA National Aeronautics and Space Administration

NOMA Non-Orthogonal-Multiple-Access

PAE Power Added Efficiency

PAPR Peak-to-Average-Power-Ratio

PMOS Positive Metal Oxide Semiconductor

PS Power Splitting

QAM Quadrature-Amplitude-Modulation

OFDM Orthogonal-Frequency-Division-Multiplexing

OFDMA Orthogonal-Frequency-Division-Multiple-Access

OOK On-Off-Keying

QoS Quality of Service

R-E Rate-Energy

RF Radio Frequency

RFID Radio Frequency Identification

RRH Remote Radio Head

SDMA Spatial-Division-Multiple-Access

SDR Semi-Definite-Relaxation

$\mathrm{SiC} \quad$ Silicon Carbide 


$\begin{array}{ll}\text { SINR } & \text { Signal-to-Interference-plus-Noise Ratio } \\ \text { SOCP } & \text { Second-Order Cone Programming } \\ \text { SS } & \text { Spatial Splitting } \\ \text { SVD } & \text { Singular-Value-Decomposition } \\ \text { SWIPT } & \begin{array}{l}\text { Simultaneous Wireless Information and Energy } \\ \text { Transfer }\end{array} \\ \text { TDMA } & \text { Time-Division-Multiple-Access } \\ \text { TS } & \text { Time Switching } \\ \text { TSMC } & \text { Taiwan Semiconductor Manufacturing Company } \\ \text { UE } & \text { User Equipment } \\ \text { UMC } & \text { United Microelectronics Corporation } \\ \text { WET } & \text { Wireless Energy Transfer } \\ \text { WIT } & \text { Wireless Information Transfer } \\ \text { WPCN } & \text { Wireless Powered Communication Network } \\ \text { WSN } & \text { Wireless Sensor Network } \\ 5 G & \text { Fifth Generation Cellular System }\end{array}$

\section{INTRODUCTION}

\section{A. Energy Shortage for Electronic Devices}

As accurately predicted by Moore's Law, the number of transistors in a dense integrated circuit doubles approximately every two years, which have been fuelling the spectacular proliferation of electronic devices since the 1960s. Furthermore, consumer electronic devices are becoming shirt-pocket-sized and mobile. Energy supply of these devices mainly comes from embedded batteries. However, as their functions become ever more sophisticated, their thirst for abundant energy is not matched by the slow progress of the batteries' capacity. The situation in the communication industry is even more daunting. Since the roll-out of the fifth-generation (5G) cellular system [1] and that of the Internet of Things (IoT) are just around the corner, people's appetite for super-high data transmission rates, for high density of connectivity and for high mobilities will indeed be satisfied to a large extent. A major portion of the future mobile data traffic will consist of novel types of services, including high-definition steroscopic video streams, augmented/virtual reality, holographic tele-presence, cloud desktops, as well as online games, etc. All these services require that the user terminals possess high computing capabilities for real-time signal processing, which may quickly drain the embedded batteries. Furthermore, sensors will be deployed in every corner of smart cities [2]. These sensors monitor the environment and upload sensing results to central servers [3]. Given the escalating number of devices involved, IoT is about to consume substantial amounts of energy [4]. However, the life-span of sensors and of sensing networks largely depends on the sensors' battery capacity. Regularly replacing the batteries may be an unrealistic and tedious task, especially in rural and low-income areas [5]. Accordingly, new sources of energy have to be explored to prolong the depletion period of conventional batteries in order to relieve the energy concerns of communication devices.

\section{B. Harvesting Energy from the Ambient Environment}

The first idea is to harvest renewable energy from the ambient environment, relying on sunlight [6], wind [7] and tidal wave [8], etc, for supporting communication devices. Since the energy arrivals are totally unpredictable, they are always modelled as stochastic processes [9]-[13]. In an idealised scenario that transmitters always have pending data packets in their buffers to be transmitted, the most challenging task is to efficiently schedule the energy consumption from the energybuffer in the face of time-varying wireless channels [14]-[18]. Furthermore, if the data packets also arrive randomly at the buffers, the mismatch between data flows and energy flows may substantially degrade the communication performance attained. For example, if excessive energy arrives at a battery without data packets to be transmitted from the data-buffer, energy gleaned may exceed the capacity of a battery, which results in the waste of energy. By contrast, if too many data packets arrive at the data-buffer without sufficient energy stored in the battery, the number of data packets may exceed the maximum length of the data-buffer, which results in packet loss. As a result, jointly scheduling both the energy queues and data packet queues is crucial for striking a balance between the energy flows and data packet flows in the time-domain. Furthermore, in a multi-user scenario, the communication devices having light data traffic load but sufficient energy are allowed to share their excess energy with their peers having a heavy data traffic load but insufficient energy. As a result, the energy may be transferred to devices having intense communication demands, which strikes a balance between energy flows and data packet flows in the spatial-domain [19][23]. Hybrid sources relying on both the renewable energy harvesters and the conventional power grid [24] may also be invoked for the sake of supplying energy for communication infrastructure [25]-[29]. But in order to reduce maintenance costs, we have to minimise the usage of the power grid.

The environment is awash with radio-frequency (RF) signals across the entire micro-wave spectrum spanning from 300 $\mathrm{MHz}$ to $300 \mathrm{GHz}$, which are emitted by TV towers, by public radio broadcast stations, by cellular base stations and by other types of RF signal generators. These RF signals constitute an abundant energy source, which can be harvested for powering communication devices [30]-[34]. Naturally, harvesting energy from RF signals is associated with different characteristics, when compared to other methods. First of all, the energy is not harvested from natural sources since the RF signals are emitted into the environment by human-made transmitters. The energy carried by RF signals is originally gleaned from conventional energy sources, such as the power grid. Harvesting energy from RF signals can be regarded as an energy recycling technique. Secondly, harvesting energy from $\mathrm{RF}$ signals does not require cumbersome energy harvesters, such as solar panels and wind/water wheels. A rectifier is sufficient for converting the alternating current carried by RF signals into direct current, which can be stored in energybuffers or can be invoked for directly driving electronic loads. As a result, RF-signal based energy harvesting is eminently suitable for powering miniature communication devices [6].

However, energy harvesting is self-initiated by communication devices that are running short of energy. Devices are only capable of passively gleaning energy from the environment, which results in a low-efficiency, in unpredictability and in uncontrolled rate of energy arrival. As a result, dedicated and controllable wireless energy transfer (WET) conceived for remotely charging numerous electronic devices have enjoyed 
an increasing research interest from both the electronic and communication engineering communities.

\section{Near-field Wireless Energy Transfer}

At the time of writing, resonant inductive coupling [35] and magnetic resonance coupling [36] emerge as flexible wireless charging options for electronic devices in the nearfield. Resonant inductive coupling based wireless charging relies on the magnetic coupling that delivers electrical energy between two coils tuned to resonate at the same frequency. This technique has already been commercialised for small electronic appliances [37], such as mobile phones, electric toothbrushes and smart watches etc. However, the coupling coils only support near-field wireless power transfer over a distance spanning from a few millimetres to a few centimetres [38], while achieving a power transfer efficiency as high as $56.7 \%$, when operating at a frequency of $508 \mathrm{kHz}$ [39]. Furthermore, resonant inductive coupling requires strict alignment of the coupling coils. Even a small misalignment may result in dramatic reduction of the power transfer efficiency [40]. As a result, during the charging process, the electronic appliances cannot be freely moved.

By contrast, magnetic resonance coupling [41] generates and transfers electrical energy between two resonators by exploiting evanescent-wave coupling. This technique has already been widely adopted for charging the electric vehicles due to its high power transfer efficiency [42]. For example, magnetic resonance coupling is capable of achieving a power transfer efficiency as high as $90 \%$ over a distance of $0.75 \mathrm{~m} \mathrm{[43].} \mathrm{Both}$ its power transfer efficiency and its charging distance are much higher than that of the resonant inductive coupling. However, magnetic resonance coupling still belongs to the category of near-field wireless charging, since its power transfer efficiency dramatically reduces to $30 \%$, when the distance is increased to $2.25 \mathrm{~m}$ [43]. Nonetheless, magnetic resonance coupling does not require strict alignment between the rechargeable device and the energy source. Hence, during the charging process, the electronic appliances may be moved within the charging area [44]. Furthermore, a multiple-input-multiple-output (MIMO) system, which has already been widely adopted for improving the performance of the wireless communication, can also be introduced into the field of magnetic resonance coupling in order to further enhance the power transfer efficiency [45] [46].

\section{RF Signal Based Wireless Energy Transfer}

In contrast to the above-mentioned pair of typical near-field techniques, the propagation of the RF signals is capable of supporting far-field WET [47]. The history of the RF signal based WET dates back to 1960, when the first long-distance wireless energy transmission was carried out by William C. Brown [48], [49]. Brown jointly designed rectifiers and antennas for energy receivers, which widely known as rectennas. They are capable of efficiently converting microwaves to direct current (DC) energy. His microwave WET system was demonstrated by powering a model helicopter from the ground in 1964 [48], [49]. In the 1970s and 1980s, intense research endeavours were invested into the RF signal based WET, which was largely motivated by the intention of developing a solar-powered satellite [50], [51]. In this system, a satellite may harvest energy from sunlight in the outer space and beam the energy back to ground stations via the RF signals. Furthermore, between 1969 and 1975, $30 \mathrm{~kW}$ of power was beamed over a distance of 1 mile at a $84 \%$ RF-DC efficiency in a project led by NASA's the Jet Propulsion Laboratory of the National Aeronautics and Space Administration (NASA) [52].

There are three main technical challenges in the RF based WET. Firstly, the long-distance propagation and adverse multipath fading may substantially attenuate the RF signals before they arrive at the receivers, which inevitably results in energy loss. Secondly, the energy carried by RF signals is of alternating-current (AC) nature, which cannot be directly invoked for driving an electronic load. As a result, RF signals have to be converted into DC energy for any further use. However, some portion of energy is inevitably lost during this conversion process. Last but not the least, the diffraction of the RF signals' waveform may expand the beam size. As a result, the receive antenna having limited size is not capable of capturing all the energy carried by the RF signals. For addressing the signal attenuation of wireless channels, the transmit beams have to be accurately aimed at the energy receivers [53], which requires the joint design of the transmit and receive antennas. For addressing the AC-DC conversion efficiency, the receive antennas have to be designed together with the rectifiers in order to achieve the impedance match for the sake of high-efficiency AC-DC conversion [54].

Compared to the near-field WET techniques, the RF signal based WET has the following advantages:

- Long coverage distance. Relying on the RF signals, energy can be transferred to receivers miles away.

- High flexibility. The angular selectivity transmit beam can be intelligently adjusted according to the energy requirements. For instance, a narrow beam can be invoked for realising accurate and high-efficiency point-to-point energy transfer, while a wide beam can be invoked for charging multiple devices in a wide area.

- More applications. RF signals can be invoked for supplying a large amount of energy to energy-hungry appliances, such as solar-powered satellite system. It can also be invoked for supplying energy to low-power devices, such as sensors and biomedical implants.

- Low investment into the infrastructure. The transmitters of the RF signals have been deployed at every corner of the globe, such as TV towers, radio broadcast stations, cellular base stations and Wi-Fi access points, etc. These transmitters can all be exploited for radiating energy to electronic devices. Only limited extra investment is required for deploying energy transmitters.

The main features of different WET techniques are summarised in TABLE I.

\section{E. WET versus WIT in the RF Spectral Band}

Since RF based WET techniques require high flexibility in terms of beam directivity for the sake of satisfying diverse charging requests, the best spectral band for steering energy 
TABLE I

Main feAtures of DifFERENT WET techniques

\begin{tabular}{|l|l|l|l|l|l|}
\hline Technique & Range & Directivity & Frequency & Antenna & Application \\
\hline Inductive coupling & Short & Low & Hz-MHz & Wire coils & $\begin{array}{l}\text { Stovetops, industrial heaters and small electric appliances, } \\
\text { such as electric toothbrush, razor and etc. }\end{array}$ \\
\hline $\begin{array}{l}\text { Magnetic resonant } \\
\text { coupling }\end{array}$ & Middle & Low & kHz-GHz & $\begin{array}{l}\text { Tuned wire coils, } \\
\text { lumped element resonators }\end{array}$ & $\begin{array}{l}\text { Portable devices, biomedical implants, electric vehicles, } \\
\text { RFID, smartcard and etc. }\end{array}$ \\
\hline RF signals & Long & High & MHz-GHz & $\begin{array}{l}\text { Parabolic dishes, rectennas, } \\
\text { phased arrays }\end{array}$ & $\begin{array}{l}\text { Solar-powered satellite, drone aircraft, IoT devices, } \\
\text { portable devices, RFID, smart cards and etc. }\end{array}$ \\
\hline
\end{tabular}

beams is in the range of $10 \mathrm{MHz}$ to $100 \mathrm{GHz}$, which almost covers all the bands allocated for wireless communication services. For instance, TV/Radio broadcasting services operate in the band spanning from $40 \mathrm{MHz}$ to $220 \mathrm{MHz}$ [55], the LTE cellular communication system operates in the spectral band spanning from $800 \mathrm{MHz}$ to $3.7 \mathrm{GHz}$ [56], while the WiFi communication system operates in the spectral band spanning from $2.4 \mathrm{GHz}$ to $6 \mathrm{GHz}$ [57]. Furthermore, as a key technique in the near future, millimetre wave ( $\mathrm{mmW}$ ) band [58] provides an attractive solution for indoor and outdoor communication services, which operates in the spectral band ranging from 10 $\mathrm{GHz}$ to $100 \mathrm{GHz}$.

WET and WIT operating in the same RF band have the following distinctive features:

- They have different functional modules. Pass-band RF signals cannot be directly invoked both for information transfer and energy harvesting. For information transfer, the pass-band RF signals have to be converted to baseband first, since all signal processing has to be completed in base-band. By contrast, for energy harvesting, the $\mathrm{AC}$ energy carried by pass-band RF signals has to be converted to DC energy first, since only DC energy can be stored in batteries or drive electronic loads.

- They require different absolute RF power at the receivers. Pasca et al. [59] have designed an RF-to-DC converter having the sensitivity of $-19 \mathrm{dBm}$ for powering wireless sensors operating in the UHF band. Moreover, Chatterjee et al. [60] have designed an RF-to-DC CMOS rectifier having a sensitivity of $-40 \mathrm{dBm}$ for energy harvesting applications. The sensitivities of the information receivers operating in GSM mode and 802.11 mode can be as low as $-104 \mathrm{dBm}$ and $-100 \mathrm{dBm}$, respectively, since the mobile devices have powerful information detection abilities. However, the miniature sensors and IoT devices have limited information detection abilities, which results in a much higher sensitivity threshold of their information receivers. For example, the state-of-the-art information receiver having a sensitivity of $-72 \mathrm{dBm}$ has been developed by Pletcher et al. [61] for wireless sensors. Furthermore, a Binary-Frequency-Shift-Keying (BFSK) based information receiver has been designed by $\mathrm{Ni}$ et al. [62] for high data rate wireless sensors, whose sensitivity is $-76 \mathrm{dBm}$ for a $6 \mathrm{Mb} / \mathrm{s}$ information transmission rate and $-90 \mathrm{dBm}$ for $500 \mathrm{~Kb} / \mathrm{s}$. As a result, the sensitivity difference between the energy harvester and the information receiver is higher than $50 \mathrm{~dB}(=-$ $40 \mathrm{dBm}-(-90) \mathrm{dBm})$.

- They cover different distances. The RF signals are atten- uated by the path-loss, shadowing and multipath fading of hostile wireless channels. Since efficient WET requires a much higher absolute energy at the receivers than WIT does, the transmission range of energy is accordingly much shorter than that of information. As a result, given the same set of transmitters and receivers, the resultant WET network has a different topology with the WIT network.

- They treat interference and noise differently. The interference and noise ubiquitously exist in any WIT system, which seriously impair the WIT performance attained. Mitigating the performance degradation imposed both by interference and noise becomes a major challenge in the design of WIT systems. By contrast, the interference and noise are in fact welcome in WET systems, since they are RF signals and they both carry useful energy. The interference and noise can be jointly processed by the energy reception modules and then they can be converted to DC energy together, which may offer additional energy harvesting gains for the energy requesters. As a result, WET systems benefit from both the interference and noise.

- They have different energy efficiency definitions. In the communication community, the energy efficiency of WIT can be defined as the ratio of information transmission throughput per $\mathrm{Hz}$ to energy consumption, which is evaluated in terms of the unit of bps/Hz/Watt or bps/Hz/Joule. Considering the classic Shannon-Hartley theorem in an Additive-White-Gaussian-Noise (AWGN) channel, the energy efficiency of WIT can be formulated as

$$
\eta_{\text {WIT }}=\frac{1}{P_{t}} \cdot \log _{2}\left(1+\frac{P_{r}}{P_{I}+P_{N}}\right) \quad(\mathrm{bps} / \mathrm{Hz} / \mathrm{Watt}),
$$

where $P_{t}$ is the transmit power of the RF signal, $P_{r}$ is the power received after the signal being attenuated by the hostile wireless channel, $P_{I}$ is the aggregate interference power and $P_{N}$ is the power of the noise at the receiver. By contrast, the energy efficiency of WET can be defined as the ratio of energy harvested by the receiver to the energy emitted by the transmitter, which can be expressed as

$$
\eta_{W E T}=\frac{1}{P_{t}} \cdot \rho\left(P_{r}+P_{I}+P_{N}\right) \quad(\text { Watt } / \text { Watt })
$$

where $\rho$ is the conversion rate from the received AC energy to the DC energy. In Fig.1, we plot the energy efficiency of WIT and that of WET, which are expressed in (1) and (2), respectively, as an example. Observe 


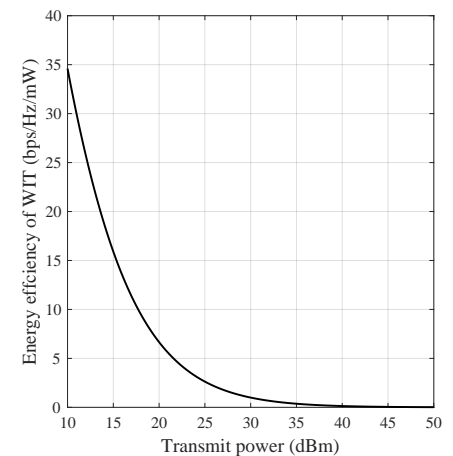

(a)

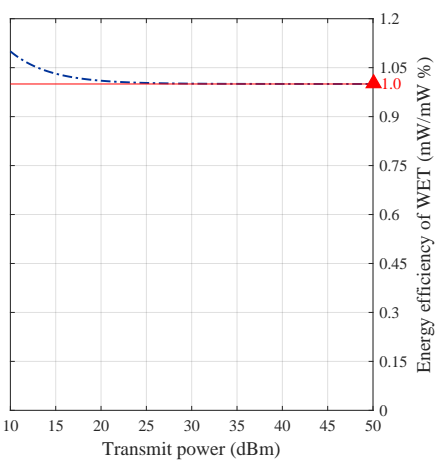

(b)
Fig. 1. Energy efficiency of WIT (a) and that of WET (b) versus transmit power of RF signals. The noise power is $P_{N}=-94 \mathrm{dBm}$, which is calculated by the power spectrum density of the thermal noise $-174 \mathrm{dBm} / \mathrm{Hz}$ and 100 $\mathrm{MHz}$ of the RF signals' bandwidth. The aggregate interference power at the receiver is set to be $P_{I}=-20 \mathrm{dBm}$, which appears in a heterogeneous cellular network with the highest probability [63]. The distance between a transmitter and receiver pair is $10 \mathrm{~m}$. The path-loss is calculated by the model invoked in [64]-[67], where the path-loss exponent is 2. No fading is assumed. The antenna gain in this example is set to be $40 \mathrm{dBi}$ in order to counteract the path-loss.

from Fig.1(a) that in our setting, the energy efficiency of WIT gradually reduces from $35 \mathrm{bps} / \mathrm{Hz} / \mathrm{mW}$ to 0 as the transmit power of the RF signal increases. By contrast, the energy efficiency of WET reduces from $1.1 \%$ but converges to $1 \%$, which is due to the channel attenuation incurred by the path-loss between the transmitter and receiver pair.

WET and WIT operating in the same RF spectral band may impair each other's performance to some extent. For example, WET requires that the RF signals carry a high power to the receivers. However, the high-power RF signals of the WET system may impose excessive interference on the WIT receivers, which may substantially degrade the WIT performance attained. As a result, coordinating WET and WIT in the same RF band imposes critical challenges on the RF circuit design, on the integrated data and energy transceiver design of the physical layer, on the resource scheduling/allocation schemes and on the corresponding protocol design of the mediumaccess-control (MAC) layer. It also requires a joint networking concept for heterogeneous data and energy transceivers. All these challenging issues require novel Integrated Data and Energy communication Networks (IDENs) [68].

\section{F. The Road to Integrated WET and WIT}

Various state-of-the-art enabling techniques can be adopted from the toolbox of wireless communications and electronic engineering for efficiently coordinating WET and WIT in the same RF spectral bands, as illustrated by the outer shell of Fig.2. The left-side of the outer shell of Fig.2 specifies techniques from the electronic engineering toolbox for enabling RF signal based WET of high-efficiency. To elaborate:

- DC-RF Energy Converters are invoked by WET transmitters for converting the DC energy gleaned from the power grid or from the embedded batteries into RF signals. For instance, it is reported in [69] that a Doherty amplifier, which is realised by a pair of voltage mode power amplifiers and transformers, is capable of achieving as high as $45 \%$ of DC-RF conversion efficiency.

- The Airy Beam was conceived as early as 1979 [70], which originated from a non-spreading Airy wave packet solution of the Schrodinger equation. The Airy beam was created and observed in both one- and two-dimensional configurations [71] and it has the following features: nondispersing, self-accelerating, self-healing and promptlyauto-focusing, which constitues as a potential solution of carrying energy to distant energy requesters. Some initial efforts [72] have been invested into generating Airy beams in the RF bands. Exploiting the compelling features of Airy beams for both the WIT and WET in the RF spectral bands calls for more research from both the electronic and communication communities.

- An accurate Impedance Matching Network is required between the receive antennas and the RF-DC converters in order to match the impedance of this pair of components [73]. Only when their impedance is accurately matched, can we suppress the energy leakage during the transfer process of RF signals from the receive antennas to the RF-DC converters.

- RF-DC Energy Converters play salient role in energy reception. Due to its AC nature, energy carried by RF signals cannot be directly invoked for charging batteries or driving electronic loads. As a result, the RF energy has to be converted into DC energy for its further exploitation. The efficiency of RF-DC energy converters is naturally a non-linear function of their input power. Suffice to say that a high RF-DC conversion efficiency can be achieved, if the input power of the converter is high [74].

- Battery Charging Circuits are capable of adapting the resultant DC signals to the specific requirements of battery charging. Specifically, after the channel's attenuation and imperfect RF-DC conversion, only a low energy reaches the battery charging circuits. Efficiently exploiting this energy and amplifying the voltage for charging batteries is an essential task of the battery charging circuits. For instance, Hammed et al. [75] designed an battery charging circuit capable of amplifying $170 \mathrm{mV}$ of input voltage to $2.8 \mathrm{~V}$ of output.

- Energy Storage Units are invoked for storing the DC energy converted from RF signals. Typically, lithium batteries are used in hand-held electronic devices for storing and supplying energy, while super-capacitors are normally embedded into miniature IoT devices. A high storage density is required by energy storage units for storing energy extracted from RF signals.

The rest of the outer shell of Fig.2 specifies the family of techniques used from wireless communication toolbox for enabling integrated WET and WIT within the same RF spectral bands:

- Constrained Channel Capacity is the terminology used for mutual information maximisation under the constraint of particular output signals. This is in contrast to Shannon's classic channel capacity theory, which aims for maximising the mutual information under the specific 


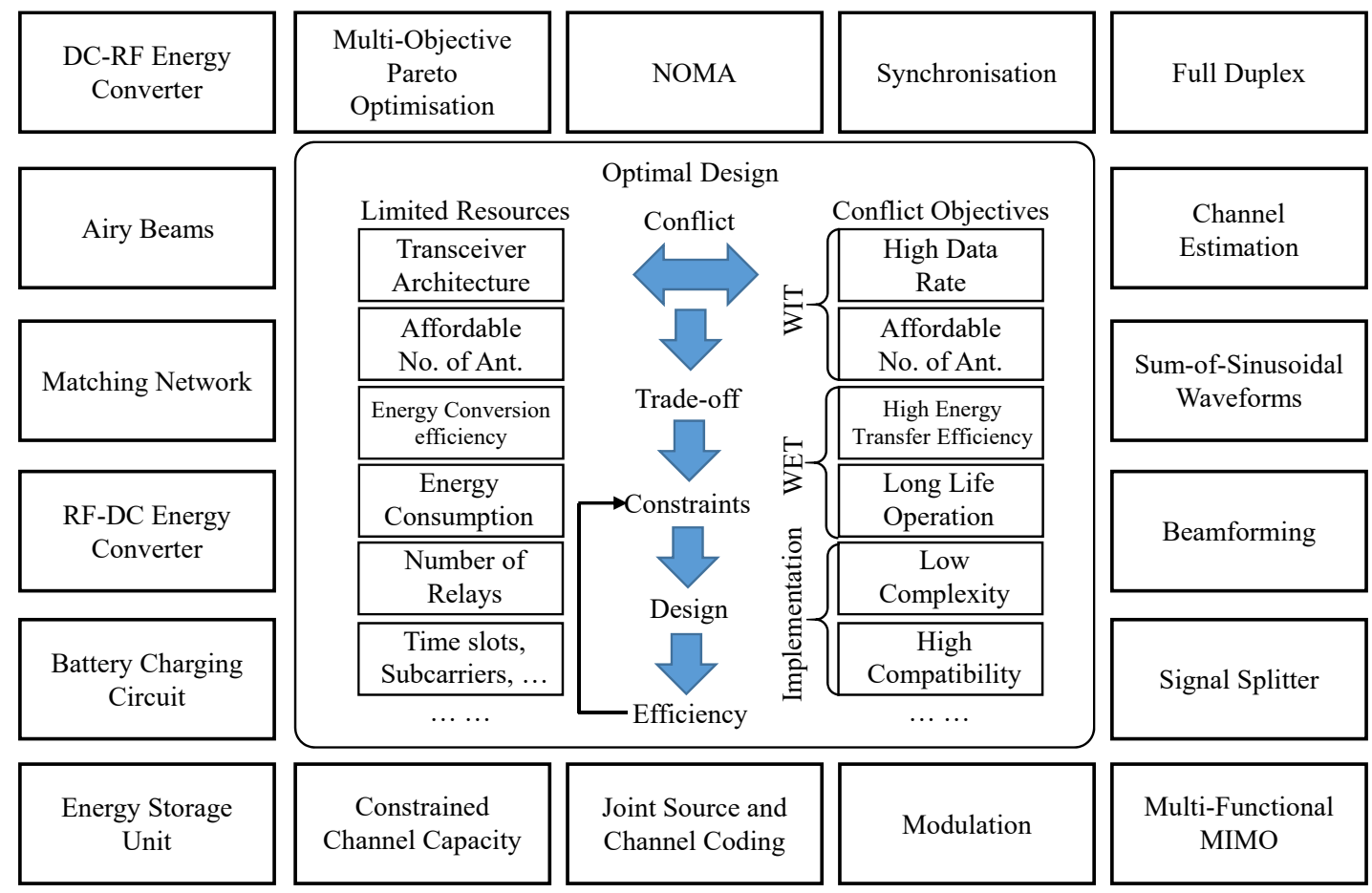

Fig. 2. Key techniques for coordinating WET and WIT in same RF spectral bands (outer shell) and optimal design principle of integrated WET and WIT (inner shell)

constraint of particular input signals. This information theoretic concept has been firstly proposed by Gastpar et al. [76] for controlling the interference in the scenario of multiple transmitter and receiver pairs. A similar methodology can be adopted here for exploring the performance limits of data transmission under the constraint of having sufficient energy carried by the output signals, which can be harvested by the receiver.

- Joint Source and Channel Coding can be specifically designed for controlling integrated WET and WIT, as proposed by Tandon et al. in [77]. Since the original messages are encoded into codewords carrying different amount of energy, joint source and channel encoders are capable of beneficially controlling the energy transfer process. Therefore, given the characteristics of the information source, a carefully designed encoder is capable of responding to energy requests, whilst simultaneously satisfying the specific data transmission requirements.

- The Modulation schemes have to be appropriately redesigned for supporting the integrated WET and WIT in IDEN. In order to reduce hardware complexity of miniature devices, the information can also be modulated onto DC signals by pulse-amplitude modulation, pulsewidth modulation and pulse position modulation routinely adopted by optical communications [78]. As a result, the RF-DC energy converter becomes capable of replacing the function of the pass-band to base-band converter. However, the integration of WET and WIT is achieved at the cost of a data transmission rate reduction, since the RF-DC converters filter the frequency and phase of the RF signals, hence we have limited choices of dimensions to be modulated for data transmission.

- Multi-Functional MIMOs are crucial for facilitating integrated WET and WIT as a benefit of their multiplexing, diversity beamforming gains [79]. Spatial multiplexing can be readily achieved by multi-functional MIMO systems in order to coordinate WET and WIT in different spatial domains, while the diversity gain attained by multiple antennas is capable of reducing the data transmission error probability, while simultaneously increasing the amount of energy transferred to the receivers. Furthermore, multi-functional MIMO systems are capable of forming narrow energy beams for supplying energy to a specific device. They are also capable of forming wide energy beams for supplying energy to multiple devices in a specific region.

- Signal Splitters are the most important modules in receiver architectures designed for integrated WET and WIT. After being received by the antennas, the RF signals are split into two portions by the signal splitters for dedicated energy and information reception. The time switcher [80] and power splitter [81] are capable of splitting the received RF signals in the time domain and power domain, respectively. The design of the signal splitter aims for avoiding energy leakage and for realising adaptive adjustment.

- Beamforming is a key technique of compensating for the energy reduction during the RF signals' propagation, which is beneficial for both the dedicated WET as well as the integrated WET and WIT. There are two basic approaches for forming a directional beam, namely analog and digital beamforming. Analog beamforming is realised 
at the transmitter by invoking an analog phase shifter for each transmit antenna for the sake of appropriately phaserotating the signal components impinging on all of the antennas. This allows us to form an angularly selective beam, hence compensating for the wireless channel's path-loss. However, the analog beamformer cannot adjust the amplitude weights of the signals transmitted by the antennas, which limits its capability of improving the performance of the dedicated WET as well as that of the integrated WET and WIT. By contrast, upon precoding the transmit symbols in the digital domain, the digital beamformer is capable of adjusting both the amplitude and the phase of the signals transmitted by the antennas. As a result, having an additional degree of freedom enables the digital beamforming to outperform its analog counterpart in terms of both the dedicated WET as well as in the integrated WET and WIT. However, as the number of the antennas becomes high, it is impractical to invoke a dedicated RF chain for each antenna. As a result, hybrid beamforming [82] is required for constructing hardwareefficient integrated WET and WIT for future massive MIMO systems.

- Sum-of-Sinusoidal type Waveforms - such as OFDMwaveforms - are capable of carrying a high amount of energy to the energy receivers [83]. Practical energy reception circuits can only be activated, if their instantaneous input power is higher than a threshold [59]. Otherwise, none of the input power can be harvested. Therefore, the waveform of the received RF signals should have a high peak-to-average-power-ratio (PAPR) for dedicated WET [84], which may be readily achieved by accumulating a number of different frequency sinusoidal signals. However, accumulating a number of different frequency sinusoidal signals requires a considerable bandwidth. By contrast, a low PAPR is required for efficient WIT. These contradicting design principles can only be resolved by radical thinking in integrated WET and WIT.

- Channel Estimation has to be carried out for efficient exploitation and support of the antenna arrangement, of beamforming and of waveform design. However, complex power-thirsty channel estimation algorithms are unsuitable for miniature IDEN devices, Hence, the design of channel estimation should be energy-friendly, requiring both low complexity and low pilot overhead. For instance, a single-bit channel-quality feedback scheme has been proposed in [85] for relieving the burden of complex channel estimation at the receivers.

- Full-duplex communications allow a transmitter to simultaneously transmit and receive data in the same spectrum, which is potentially capable of doubling the bandwidth efficiency. The full duplex concept can also be exploited for supporting integrated data and energy transfer. For instance, in [86], an IDEN-station was advocated, which is capable of transferring energy in the downlink and receiving data in the uplink. However, since WET normally requires a high transmit power, the self-interference imposed by the downlink WET on the uplink WIT has to be carefully dealt with. By contrast, the transmitter is also capable of transferring data in the downlink and receiving energy in the uplink. In this case, the self-interference can be recycled as an additional energy source for beneficially exploiting the energy emitted by the transmitter in [87].

- Synchronisation constitutes an essential part of all communications systems [88] and it typically entails establishing coarse initial synchronization of both the symbol timing as well as of the carrier frequency of the transmitter and the receiver. Both the wireless channel as well as the oscillator's carrier phase offset impose undesirable phase rotation and hence a potential loss of synchronization between the transmitter and the receiver, which hence requires accurate tracking. Furthermore, the WET signals and WIT signals may be interleaved with each other in the time domain. The receiver has to precisely control the timing of switching between the information decoding mode and the energy harvesting mode in order to recover the information requested from the WIT signals, while harvesting the requested amount of energy from the WET signals [89].

- Non-Orthogonal-Multiple-Access (NOMA) is a popular technique, which is capable of offering higher bandwidth efficiency than the orthogonal-frequency-divisionmultiple-access (OFDMA) and the time-divisionmultiple-access (TDMA) techniques. Although the superimposed RF signals dedicated to different user equipments (UEs) may impose a high complexity on the receivers, constructively combining RF signals in a non-orthogonal manner is capable of beneficially concentrating the energy carried by the resultant RF signals. As a result, the efficiency of WET can be substantially improved. However, the accurate estimation of amplitude attenuation and phase shift of the channel is a prerequisite for constructively combining the $\mathrm{RF}$ signals at the transmitters.

- Multi-Objective Optimisation is also beneficial in terms of simultaneously satisfying the demands of both WET and WIT. IDENs have to provide both data and energy services for the UEs. Multi-component objective functions have to be optimised in the design of all layers. However, finding the Pareto front ${ }^{1}$ of the multi-objective optimisation requires substantial computing capabilities. The emerging quantum computing technique may efficiently solve multi-objective Pareto-optimisation problems. For instance, a powerful quantum computing aided algorithm has been invoked for simultaneously minimising energy consumption and achieving load balancing in the routing design of ad hoc wireless networks [90].

Awareness of diverse types of contextual information [91], such as the specific activities of the devices, the geographic location information, the battery levels, the energy consumptions and the channel coefficients, is essential for the efficient design of the integrated WET and WIT. The inner shell of Fig.2 summarises generic optimal design principles to be obeyed for coordinating WET and WIT in the same spectral

\footnotetext{
${ }^{1}$ The Pareto front is the collection of these solutions, where none of the OF components can be improved without degrading one orseveral of the others.
} 
band. Overall, in the design of integrated WET and WIT, the fundamental conflict between the limited resources and unlimited quality of service (QoS) requirements have to be addressed. For instance, in an IDEN system, the functionalities of the integrated data and energy transceivers are limited by their hardware. Only a limited number of antennas can be implemented, which results in limited diversity, multiplexing and beamforming gains. Imperfect electronic modules of the transceivers suffer from considerable energy leakage and insufficient energy conversion. Only limited resources can be provided for integrated WET and WIT in the time domain, frequency domain, power domain and in the spatial domain. However, a potentially unlimited QoS has to be satisfied in IDEN. Specifically, WIT is always expected to achieve a high data rate, low transmission latency, while WET aims for achieving a high energy transfer efficiency for extending the battery recharge-period of electronic devices. Additionally, the implementation of integrated WET and WIT requires a low complexity and a high compatibility. Furthermore, conflicts also exist between the different QoS requirements. For example, since both WIT and WET is supported by RF signals, they have to compete for limited resources for the sake of satisfying their QoS requirements. As a result, we have to strike a balance between the performance of WET and WIT. An integrated WET and WIT system of high performance also requires complex hardware implementation. We also have to carefully consider the tradeoff between the achievable performance and the implementation complexity for arriving at an attractive integrated WET and WIT.

\section{G. Contributions and Organisations}

There have been several outstanding surveys and tutorials in the related research topics, such as wireless charging and energy harvesting aided communication. Specifically, Niotaki et al. [92] have investigated solar/electromagnetic energy harvesting and wireless power transmission, covering the design of hybrid solar-electromagnetic harvesters, the circuits of direct current combiners and the efficient solar-toelectromagnetic converters. Furthermore, novel circuit topologies were presented for the sake of minimising the sensitivity of rectifier circuits. Lu et al. [93] have summarised the latest progress in both near-field inductive coupling and in magnetic resonance based wireless charging techniques as well as in far-field RF based WET techniques. Moreover, the technical standards of wireless charging and their recent advances in networked applications were also investigated. However, both [92] and [93] were mainly focused on circuit design for incorporating wireless charging into electronic devices, but they did not consider the integration of wireless charging and wireless communication.

Apart from the existing survey and tutorials on WET, some further endeavours [94]-[96] have been invested in characterising the landscape of energy harvesting aided communication. Specifically, Ulukus et al. [94] have summarised the state-ofthe-art in wireless networks relying on energy harvesting aided communication nodes, including their information theoretic performance limits, transmission scheduling policies, resource
TABLE II

MAIN FEATURES OF RELATED SURVEYS AND TUTORIALS

\begin{tabular}{|c|l|l|}
\hline Ref. & Topic & Contribution \\
\hline$[92]$ & $\begin{array}{l}\text { Energy } \\
\text { harvester } \\
\text { design }\end{array}$ & $\begin{array}{l}\text { Investigate the advanced hybrid solar-electromag- } \\
\text { netic harvesters, direct current combiners, and } \\
\text { solar to electromagnetic converter. }\end{array}$ \\
\hline$[93]$ & $\begin{array}{l}\text { Wireless } \\
\text { charging }\end{array}$ & $\begin{array}{l}\text { Review near-field/far-field WET techniques, their } \\
\text { technical standards and their recent advances in } \\
\text { network application. }\end{array}$ \\
\hline$[94]$ & $\begin{array}{l}\text { Energy } \\
\text { harvesting } \\
\text { comm./net. }\end{array}$ & $\begin{array}{l}\text { Summarise wireless networks consisting of ener- } \\
\text { gy harvesting nodes, including performance lim-, } \\
\text { it, transmission scheduling, resource allocation, } \\
\text { medium access control and networking issues. }\end{array}$ \\
\hline$[95]$ & $\begin{array}{l}\text { RF Energy } \\
\text { harvesting } \\
\text { comm./net. }\end{array}$ & $\begin{array}{l}\text { Review circuit design of RF energy harvester, } \\
\text { receiver architecture, resource allocation as well } \\
\text { as protocol design in RF energy harvesting aid- } \\
\text { ed wireless networks. }\end{array}$ \\
\hline
\end{tabular}

allocation, medium access control and networking issues. Additionally, the energy cooperation between communication nodes as well as the energy harvesting and energy consumption models have also been reviewed. Another comprehensive survey [95] has been contributed by $\mathrm{Ku}$ et al., dealing with the challenges incurred by the intermittent random nature of the renewable energy arrival for improving energy harvesting aided communication systems. Furthermore, Lu et al. [96] have focused their attention on specific RF energy harvesting techniques and on their integration with wireless communication. Both the associated circuits, the receiver architecture as well as the resource allocation and protocol designs have been reviewed in the context of RF energy harvesting aided wireless network design. However, energy harvesting is an operation adopted by the devices for acquiring additional energy from the environment, hence these devices can be regarded as passive energy receivers. As a result, scheduling and balancing energy and data flows of stochastic nature become the main challenge in energy harvesting aided communication. More specifically, RF energy harvesting aided wireless communication is mainly concerned with the operating strategy adopted by the receivers. For instance, the receivers may invoke efficient RF-DC converters for increasing the amount of energy harvested from RF signals. They may also decide how to split the received signals for simultaneously satisfying both the data and energy demands. However, RF energy harvesting aided wireless communication and networking ignores the evaluation and design of the entire RF based WET chain and it does not consider the joint design of careful integrated data and energy transmitters and receivers. The main features of the above literature have been summarised in TABLE II.

Against the above-mentioned literature, our contribution aims for summarising the latest progresses on IDEN-related research and for shedding light on the design of the complete integrated data and energy transfer chain. The contributions and the organisation of our treatise are summarised below:

- In Section II, we will introduce the ubiquitous architecture of the emerging IDENs by summarising its heterogeneity and by synthesising a diverse range of WIT and WET scenarios, which includes integrated data and energy transfer, wireless powered communication, pointto-point wireless charging and multicast wireless charging. Our vision concerning the potential functionalities of 
IDENs is also outlined.

- In Section III, We will then reveal the inherent relationship between WIT and WET from an information theoretical perspective. The basic methodology of deriving rate-energy functions is elaborated on for both discrete and continuous channel input and output. Some initial results are provided for classic binary channels and for the AWGN channel.

- In Section IV, we will detail the entire circuit chain conceived for enabling RF-signal based WET, including the DC-RF circuit design of the energy transmitters, the propagation of RF signals in wireless channels for WET, the RF-DC circuit design at the energy receivers and the charging circuits designed for storing energy in the batteries.

- In Section V, we will also highlight the transceiver design of the physical layer for IDENs operating in the RF spectral band, where both MIMO aided transmitter and receiver architectures designed for IDENs are illustrated. Furthermore, this treatise also takes the waveform design into account.

- In Section VI, we will elaborate both on the resource allocation and on the protocol design of the medium access control in IDENs. Popular resource allocation algorithms proposed for integrated data and energy transfer are reviewed in the context of both centralised noncooperative and in cooperative cellular networks. The medium access control protocols of distributed networks, such as wireless local area networks (WLANs) and sensor networks, are also introduced.

- In Section VII, we will summarise the latest contributions in both the centralised and distributed networking techniques designed for IDENs. Furthermore, a range of practical application scenarios of IDENs are also thoroughly reviewed.

- Other open challenges of IDENs are listed in Section VIII, while our conclusions are offered in Section IX.

The outline of our treatise is provided at a glance in Fig.3.

\section{Ubiquitous Architecture of Heterogeneous IDENs}

IDENs are naturally heterogeneous in terms of all their technical aspects. We will investigate the heterogeneity of a typical IDEN and synthesise a diverse range of WET and WIT scenarios into its ubiquitous architecture, which is exemplified in Fig.4.

\section{A. Heterogeneous Infrastructure}

First of all, various types of infrastructure elements coexist in heterogeneous IDENs. As portrayed in Fig.4, there are generally three basic infrastructure types in IDENs, namely IDEN stations, WET stations and WIT stations/relays.

IDEN stations [97] are capable of operating both as information transmitters and as energy transmitters for satisfying UEs' data and charging requests. Due to their powerful functionalities, IDEN stations are also capable of realising integrated data and energy transfer in order to increase the spectrum efficiency of the congested RF band. Therefore, IDEN stations

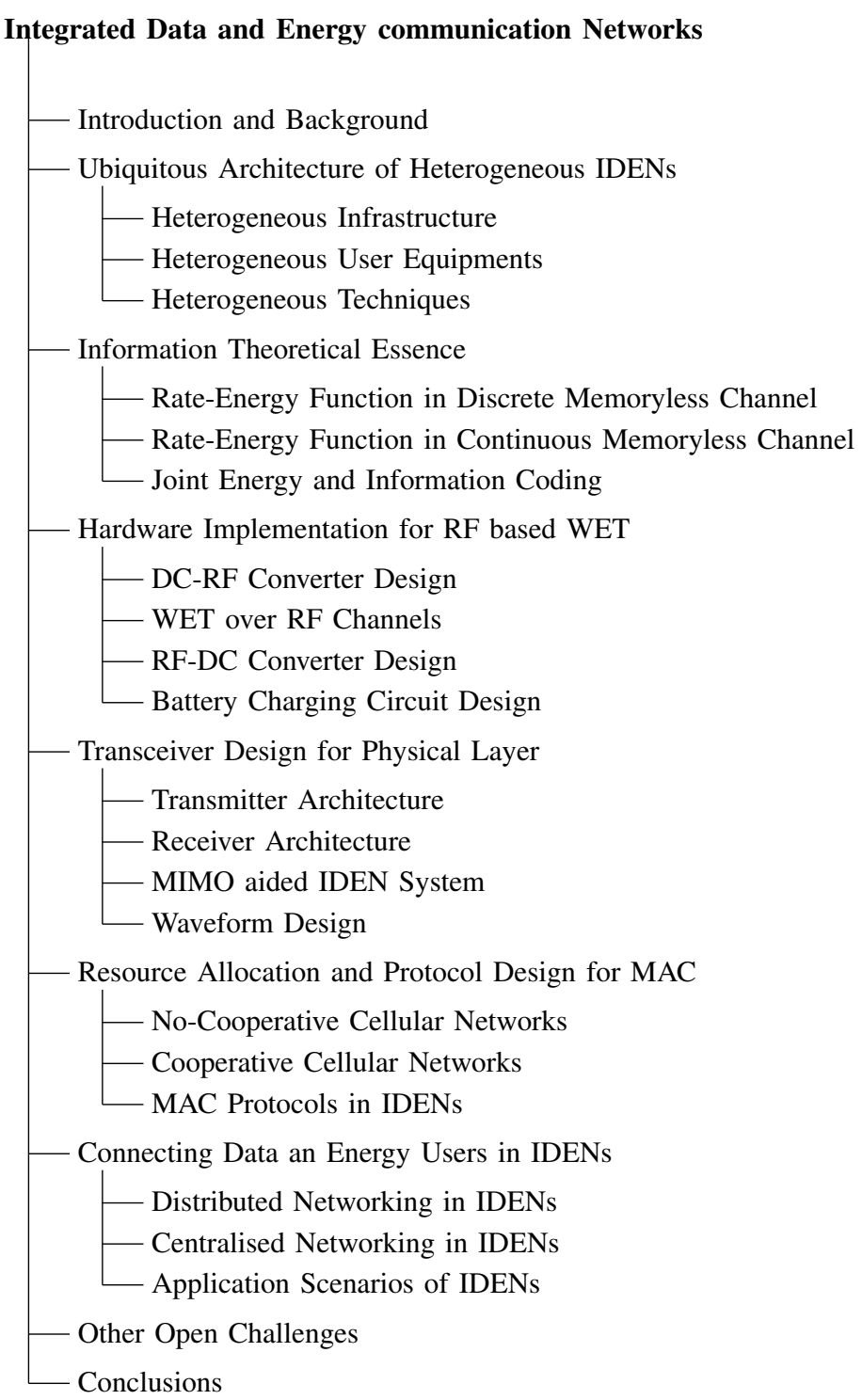

Fig. 3. The outline of this treatise

have to be connected to the core communication network and they also have to be connected to stable energy sources, such as large solar energy harvesters and the power grid. For instance, IDEN-Station-1 may satisfy the integrated data and energy request of IDEN-UE-1 and that of the IoT devices, as illustrated in Fig.4.

However, as we briefly discussed in Section I-E, the reliable reception range of the WET is far shorter than that of the WIT, as shown in Fig.4. As a result, some blind spots cannot be adequately covered by the WET of IDEN stations. Therefore, some WET stations [98] are also deployed in order to supply energy to the devices roaming in these blind areas. These WET stations are only connected to energy sources, but they do not have to be connected to the core communication network. As a result, they are dedicated for satisfying the UEs' charging requests. For instance, as shown in Fig.4, three WET stations are deployed in order to supply energy to UEs beyond the WET ranges of the IDEN stations.

Apart from IDEN stations and WET stations equipped with 


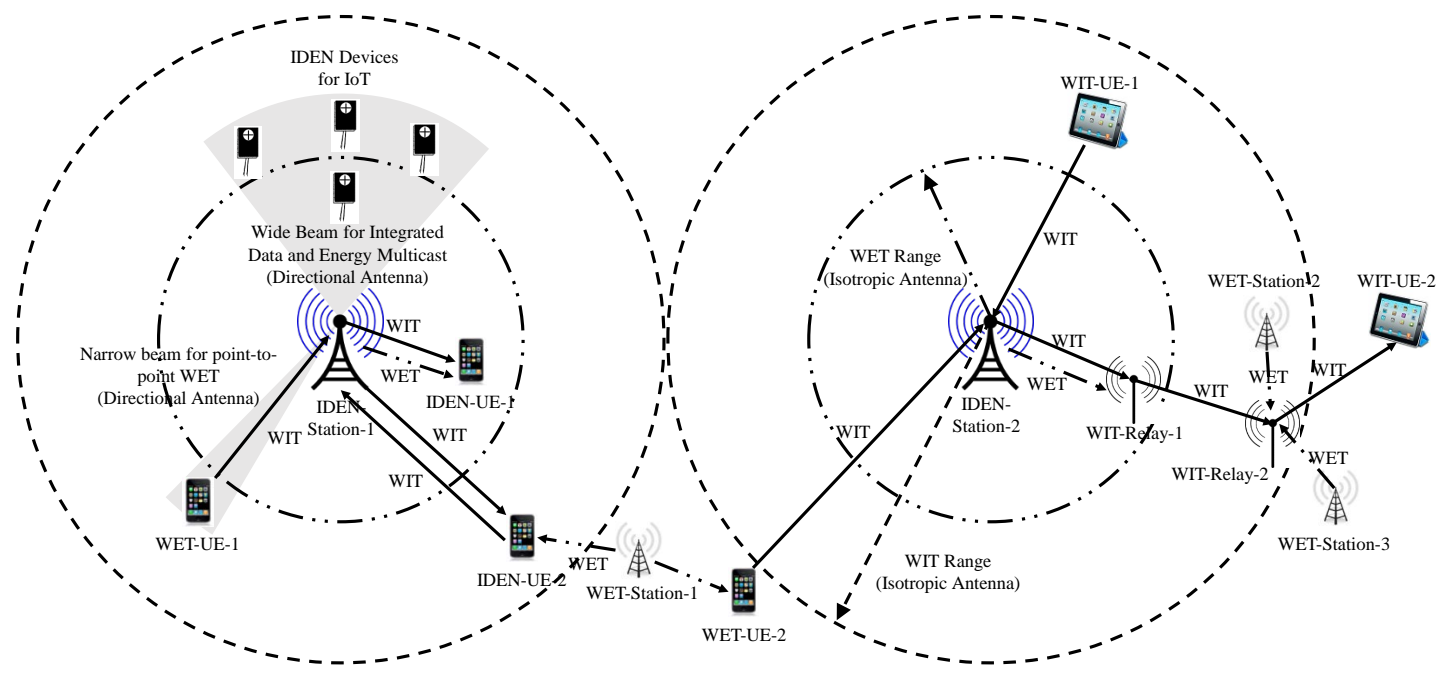

Fig. 4. Ubiquitous Architecture of a Heterogeneous IDEN

the WET function, there are also some dedicated WIT stations in heterogeneous IDENs, such as the classic macro-cellular, pico-cellular and femto-cellular base stations [99]. These communication stations have different levels of transmit power and different coverage, which results in obvious heterogeneity in IDENs. Sometimes, low-cost relay stations are also deployed for forwarding the data packets to cell-edge users, as illustrated in Fig.4. However, small cellular stations and relay stations [100] are only capable of emitting RF signals at a limited power. They are not suitable for carrying out sophisticated WET tasks. As a result, they are conceived as dedicated communication infrastructure elements.

\section{B. Heterogeneous User Equipment}

Apart from their heterogeneous infrastructure, IDENs have to accommodate both communication and charging requests from different types of UEs. We generally have three types of UEs in heterogeneous IDENs, namely WIT UEs, WET UEs and IDEN UEs [101].

WIT UEs only require downlink and uplink data transmissions in IDENs. Since the operation of these UEs is always supported by stable energy sources, they do not have to ask for expensive wireless charging from IDENs. Laptops and tablets are typical WIT UEs, since they are either equipped with high-capacity batteries or connected to the power grid for acquiring sufficient energy. For example, as illustrated in the right cell of Fig.4, WIT-UE-1 may consume its own energy for supporting its uplink data transmission, while WIT-UE-2 receives its requested data from IDEN-Station- 2 with the aid of two WIT relay stations.

By contrast, WET UEs have to request additional energy supply from either the IDEN stations or from the WET stations in order to support their basic functionalities, such as uplink data transmissions and energy-consuming computations, since they are not connected to stable energy sources [86]. For example, WET-UE-1 is charged by IDEN-Station-1. This energy is then consumed by WET-UE-1 for its uplink WIT transmissions, as shown in the left cell of Fig.4. Similarly, al- though WET-UE-2 is beyond the WIT range of IDEN-Station2 , it may still establish uplink WIT transmissions with IDENStation-2 by exploiting the additional energy received from WET-Station-1. Battery-powered user terminals are typical WET UEs, since their functionalities are limited by the amount of energy stored in their batteries.

There are also UEs simultaneously requesting data transmission and wireless charging, which are regarded as IDEN UEs [102]. For instance, in the left cell of Fig.4, IDEN-UE1 simultaneously receives its requested data and energy from the RF signal emitted by IDEN-Station-1. IDEN-UE-2 also simultaneously requests both downlink data transmission and wireless charging. However, since it is beyond the WET range of IDEN-Station-1, IDEN-UE-2 can only receive its requested data from IDEN-Station-1, while receiving energy from WETStation-1. This energy may be exploited for supporting IDENUE-2's uplink data transmission to IDEN-Station-1.

Sometimes, the functionalities of WIT relay stations are also limited by their energy supply, especially when the WIT relay stations rely on energy gleaned from batteries or harvested from renewable sources. As a result, they also need wireless charging from IDEN stations or WET stations for powering their data packet forwarding [103]. In this sense, WIT relay stations can also be regarded as "IDEN UEs". As exemplified in the right cell of Fig.4, both data and energy are simultaneously transferred from IDEN-Station-2 to WIT-Relay-1. The energy received by WIT-Relay-1 may be further exploited for forwarding the data packets to the next hop. Since WITRelay-2 is beyond the WET range of IDEN-Station-2, it has to request energy transfer from the nearby WET-Station-2 and WET-Station-3. After receiving the data packets from WITRelay-1 and gaining sufficient energy from the WET stations, the data packets are finally forwarded to their destination WITUE-2.

\section{Heterogeneous Techniques for WIT and WET}

Although WET and WIT may operate in the same RF spectral band, they have distinctive characteristics according 
to our discussions in Section I-E. As a result, the coexistence of WET and WIT results in natural heterogeneity to IDEN for satisfying the UEs' charging requests and data transmission requests.

If we further look into data transmission actions in IDENs, various WIT techniques are invoked. As shown in Fig.4, the boundary of an IDEN cell is determined by the WIT range of an IDEN station, when isotropic antennas are used. Therefore, any UEs within the WIT range of an IDEN station may receive its requested data via a single-hop cellular link, while they may also upload information to their associated IDEN stations without any energy aid. For instance, IDEN-UE-1, IDEN-UE2 and WIT-UE-1 all receive their requested data from their associated IDEN stations, while WET-UE1 and IDEN-UE2 both upload their data towards IDEN-Station-1, as shown in Fig.4. By contrast, if a UE is beyond the WIT range of an IDEN station, multiple relay stations have to be invoked for relaying data from an IDEN station to the requesters or in a reverse direction via multi-hop transmissions, such as the downlink transmission from IDEN-Station-2 to WIT-UE-2, which is illustrated in Fig.4. Additionally, relying on the extra energy supplied by WET stations, a UE beyond the WIT range of an IDEN station is also capable of uploading data to the IDEN station [86], such as the uplink transmission of WETUE-2 in Fig.4, which is assisted by WET-Station-1.

Moreover, different WET techniques have to be invoked for satisfying diverse charging request. As portrayed in Fig.4, the WET range of an IDEN station is much shorter than its WIT range, when isotropic antennas are used. This is because WET requires high absolute energy for activating the energy harvesting circuit. Hence, WET is more sensitive to the signal propagation attenuation, which is dominated by the path-loss. The UEs within this WET range are capable of receiving energy from IDEN stations. By contrast, when WET UEs move beyond the WET range of an IDEN station, they have to request energy from their nearby WET stations. If directional antennas are used by the IDEN stations, they may also supply sufficient energy to WET UEs beyond their limited WET ranges determined by conventional isotropic antennas, which is achieved by forming dedicated narrow-angle energy beams towards these WET UEs [104]. As shown in the left IDEN cell of Fig.4, although WET-UE-1 is outside IDEN-Station1's WET range, it is still capable of receiving energy from the dedicated narrow beam coming from IDEN-Station-1.

Furthermore, Internet of Things (IoT) devices will be pervasively deployed in the near future. Our heterogeneous IDENs are also responsible for satisfying both of their communication and energy demands. IoT devices normally are clustered in a specific area in order to jointly carry out their tasks. As a result, in order to fulfil the energy requests of multiple devices, IDEN stations may form wide-angle energy beams for covering the group of requesters. This technique may be regarded as energy multicast [105]. Furthermore, this wide beam is also capable of carrying information and energy together in order to multicast the integrated data and energy to the requesters.

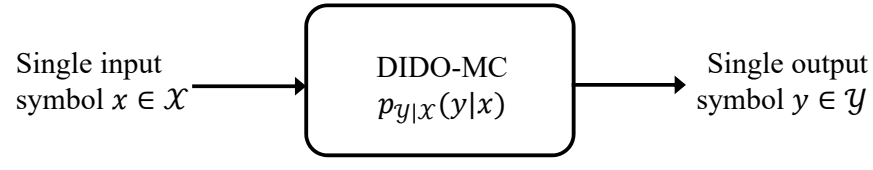

Fig. 5. Discrete-Input-Discrete-Output Memoryless Channel

\section{Information Theoretical Essence For IDEN}

As previously discussed in Section I-E, the WET and the WIT entail several conflicting specifications, when they are coordinated in the same RF spectral band. As a result, theoretical investigations have to be carried out in order to reveal the underlying relationship between the WET and WIT in IDENs, which may provide researchers and engineers with further valuable insights on improving the system-level performance of IDENs. In this section, we will explore the information theoretical essence for integrated WET and WIT.

Note that the information theoretical exploration remain valid not only for the integrated WET and WIT operating in the RF spectral band, but also for all other integrated informatioion and energy transfer scenarios, such as the power line communication [106] and the power over Ethernet technique [107]. Classic information theoretical channel capacity analysis has been dedicated to maximising the mutual information under the constraint of specific input signals. By contrast, the pioneering work of Gastpar [76] has attempted to maximise the mutual information under the constraint of specific output signals, which aims for controlling the interference imposed on a communication pair by other peers. This piece of work may provide us with valuable hints for finding the performance limits of integrated information and energy transfer.

\section{A. Discrete-Input-Discrete-Output Memoryless Channel}

We first consider the classic Discrete-Input-Discrete-Output Memoryless Channel (DIDO-MC) of Fig.5. If a DIDO-MC has a single input symbol $x$ and a single output symbol $y$, the transition probability of this DIDO-MC channel may be expressed by the probability function of $p_{y \mid X}(y \mid x)$. All the legitimate values of the input symbol $x$ constitute the input codebook $\mathcal{X}$, while all the legitimate values of the output symbol $y$ constitute the output codebook $\mathcal{Y}$. Furthermore, given a specific output symbol $y$, the energy carried by it can be represented by the non-negative function $g(y)$.

Let us now move on to the $n$-dimensional random input, which is expressed by the vector $\mathbf{X}^{n}=\left(X_{1}, X_{2}, \cdots, X_{n}\right)$. All the random symbols in the vector $\mathbf{X}^{n}$ are independent of one another. If a sample of the $n$ dimensional random input is $\mathbf{x}^{n}=$ $\left(x_{1}, x_{2}, \cdots, x_{n}\right)$, its corresponding occurrence probability can be expressed as $p_{X^{n}}\left(\mathbf{x}^{n}\right)=\prod_{i=1}^{n} p_{\mathcal{X}}\left(x_{i}\right)$, where $\mathcal{X}^{n}$ represents the $n$-dimensional codebook containing all the possible values of the random input $\mathbf{X}^{n}$ and $p_{X}\left(x_{i}\right)$ represents the probability of the symbol $x_{i}$ being generated. When the input sample $\mathbf{x}^{n}$ is transmitted by the information source, the corresponding output at the information destination is denoted by the vector $\mathbf{y}^{n}=\left(y_{1}, y_{2}, \cdots, y_{n}\right)$. This sequence of symbols occurs with a probability of $p_{\boldsymbol{y}^{n}}\left(\mathbf{y}^{n}\right)=\prod_{i=1}^{n} p_{y}\left(y_{i}\right)$, where $\boldsymbol{y}^{n}$ represents the 


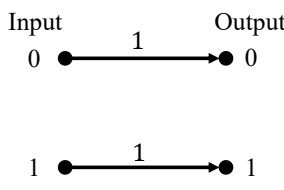

(a) Noiseless Channel

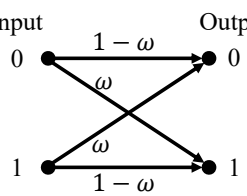

(b) Symmetric Channel

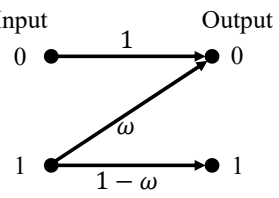

(c) Z Channel
Fig. 6. Three typical binary channels

$n$-dimensional codebook containing all the possible values of the random input $\mathbf{Y}^{n}$ and $p_{y}\left(y_{i}\right)=\sum_{x_{i} \in X} p_{\mathcal{X}}\left(x_{i}\right) p_{\mathcal{Y} \mid X}\left(y_{i} \mid x_{i}\right)$ represents the probability of the symbol $y_{i}$ being received by the information destination. The energy carried by the sequence $\mathbf{y}^{n}$ can be calculated by the non-negative function $g\left(\mathbf{y}^{n}\right)$. Assuming a random output sequence $\mathbf{Y}^{n}=\left(Y_{1}, Y_{2}, \cdots, Y_{n}\right)$, the average energy carried by this $n$-dimensional sequence can be formulated as $\mathbb{E}\left[g\left(\mathbf{Y}^{n}\right)\right]=\sum_{\mathbf{y}^{n} \in \boldsymbol{Y}^{n}} g\left(\mathbf{y}^{n}\right) \cdot p_{\mathbf{y}^{n}}\left(\mathbf{y}^{n}\right)$. As a result, the tradeoff between the information transfer and the energy transfer can be formulated as the following optimisation problem:

$$
\begin{aligned}
& \text { Objective: } \max _{p_{X^{n}}\left(\mathbf{X}^{n}\right)} I\left(\mathbf{X}^{n} ; \mathbf{Y}^{n}\right), \\
& \text { Subject to: } \frac{1}{n} \cdot \mathbb{E}\left[g\left(\mathbf{Y}^{n}\right)\right] \geq \beta,
\end{aligned}
$$

where $I\left(\mathbf{X}^{n} ; \mathbf{Y}^{n}\right)$ is the average mutual information between the $n$-dimensional input symbol sequence and its output counterpart. Given the transition probabilities $p_{\mathcal{Y} \mid X}(\mathbf{Y} \mid \mathbf{X})$ of the DMC, the optimisation problem (3) aims for finding the optimal $n$ dimensional information source, which is represented by the probabilities $p_{\mathcal{X}^{n}}\left(\mathbf{x}^{n}\right)$ of the symbol sequences being generated. The constraint ( $3 a$ ) suggests that the average energy carried by a single output symbol has to be higher than a threshold $\beta$ in order to satisfy the energy transfer requirement. Substituting the optimal $p_{\mathcal{X}^{n}}\left(\mathbf{x}^{n}\right)$ into the objective (3), we may derive the channel capacity $C_{n}(\beta)$, when the input is an $n$-dimensional symbol sequence. Note that $C_{n}(\beta)$ is a function of the energy constraint $\beta$. Furthermore, the normalised channel capacity subject to the energy constraint $\beta$ can be further formulated as

$$
C(\beta)=\sup _{n} \frac{1}{n} \cdot C_{n}(\beta), \quad[\mathrm{bit} / \mathrm{symbol}],
$$

which may be regarded as the rate-energy function. Observe from (4) that the rate-energy function is a natural extension of the classic channel capacity concept. This function only depends on a channel's statistical property and on the requirement of the energy transfer, but it does not rely on the information source.

The rate-energy functions of some simple binary channels will now be studied for illustrating the above information theoretical methodology. In binary channels, the energy carried by the output symbol can be defined as follows. If the output symbol is " 0 ", its energy is $g(0)=0$. If the output symbol is " 1 ", its energy is $g(1)=1$.

As illustrated in Fig.6(a), in a noiseless channel, the binary symbol " 0 " or " 1 " can be delivered from the information source to the destination without any distortion. By exploiting the maximum entropy theory of the information source, the closed-form rate-energy function of the noiseless channel can be expressed as [108]

$$
C(\beta)= \begin{cases}\log 2, & 0 \leq \beta \leq \frac{1}{2}, \\ H_{2}(\beta), & \frac{1}{2}<\beta \leq 1,\end{cases}
$$

where $H_{2}(\beta)=-\beta \log \beta-(1-\beta) \log (1-\beta)$ is the binary entropy function with respect to $\beta$.

As portrayed in Fig.6(b), in a symmetric channel, the probabilities of the input symbols " 0 " and " 1 " being delivered to the destination without any distortion are both $\omega$, while the probability of the input symbol 1 being converted to the output symbol 0 and the probability of the input symbol 0 being converted to the output symbol "1" are both $(1-\omega)$. The closed-form rate-energy function of the symmetric channel can be formulated as [108]

$$
C(\beta)= \begin{cases}\log 2-H_{2}(\omega), & 0 \leq \beta \leq \frac{1}{2} \\ H_{2}(\beta)-H_{2}(\omega), & \frac{1}{2}<\beta \leq 1-\omega,\end{cases}
$$

where $H_{2}(\beta)$ and $H_{2}(\omega)$ are binary entropy functions with respect to $\beta$ and $\omega$, respectively. According to classic information theory without any consideration of WET, the average mutual information can be maximised when the binary symbols generated by the binary information source have equal probabilities. In this case, the maximum energy delivered to the information destination is only $\frac{1}{2}$ unit in average. If the energy threshold at the destination is higher than $\frac{1}{2}$, the information source has to increase the probability of transmitting symbol " 1 ". If the source only transmits symbol "1", the destination may receive the maximum energy $\beta_{\max }=(1-\omega)$.

As shown in Fig.6(c), in a $\mathrm{Z}$ channel, the input symbol " 0 " can be delivered to the destination without any error probability. However, the input symbol " 1 " may be converted to the output symbol "0" with a probability of $\omega$, while it remains the same at the output with a probability of $(1-\omega)$. The closed-form rate-energy function of the $\mathrm{Z}$ channel can be formulated as [108]

$$
C(\beta)= \begin{cases}\log \left(1-\omega^{\frac{1}{1-\omega}}+\omega^{\frac{\omega}{1-\omega}}\right), & 0 \leq \beta \leq(1-\omega) \pi^{*} \\ H_{2}(\beta)-\frac{\beta}{1-\omega} H_{2}(\omega), & (1-\omega) \pi^{*}<\beta \leq 1-\omega,\end{cases}
$$

where the variable $\pi^{*}$ is given by [108]

$$
\pi^{*}=\frac{\omega^{\frac{\omega}{1-\omega}}}{1+(1-\omega) \omega^{\frac{\omega}{1-\omega}}}
$$

We plot the rate-energy functions of these binary channels in Fig.7. Observe from Fig.7 that given the same energy threshold $\beta$, naturally the noiseless channel has the highest information rate, since the information symbols can be transmitted without any error probability over this channel. Furthermore, we also find that given the same energy threshold $\beta$, the $\mathrm{Z}$ channel may achieve a higher information rate than the symmetric channel, since the $\mathrm{Z}$ channel produces fewer errors than the symmetric channel.

\section{B. Continuous-Input-Continuous-Output Memoryless Channel}

A similar methodology can also be applied in a continuousinput-continuous-output memoryless channel (CICO-MC), as shown in Fig.8. Both the input message and the output 


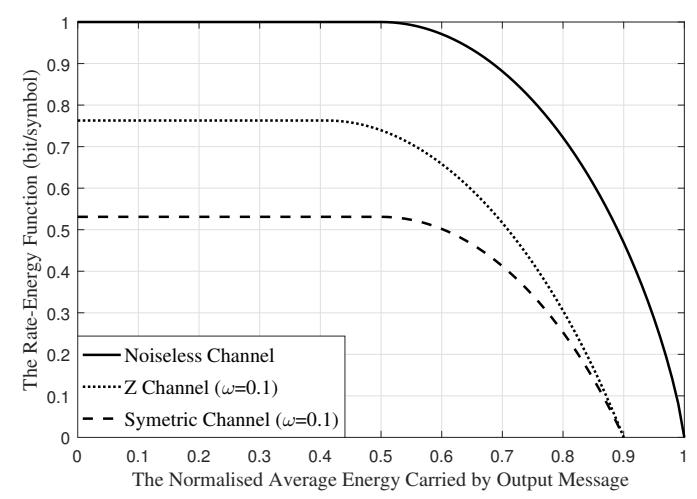

Fig. 7. Rate-energy functions for three binary channels

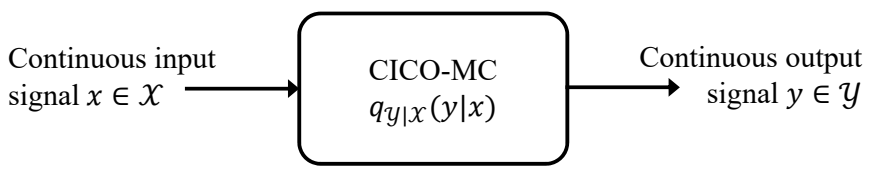

Fig. 8. Continuous-Input-Continuous-Output Memoryless Channel

message are continuous random variables, defined as $X$ and $Y$ respectively. The transition probability density function of a CICO-MC can be defined as $q_{Y \mid X}(y \mid x)$, when the information source randomly generates the continuous input message $x$ and the corresponding continuous output message $y$ is received by the information destination. All the legitimate values of the random input $X$ constitute the space $\mathcal{X}$, while the space $\mathcal{Y}$ contains all the legitimate values of $Y$. The energy carried by a continuous output message $y$ can be represented by the function $g(y)$. Given the transition probability density function $q_{Y \mid X}(y \mid x)$ of the CICO-MC, the average energy $\mathbb{E}[g(y)]$ is dependent on the cumulative distribution function $F_{X}(x)$ of the continuous random input $X$.

Therefore, the rate-energy function of the CICO-MC can be formulated by the following optimisation problem:

$$
\begin{aligned}
& \text { Objective: } \max _{F_{X}(x)} I(X ; Y), \\
& \text { Subject to: } \mathbb{E}[g(y)] \geq \beta,
\end{aligned}
$$

where $I(X ; Y)$ is the average mutual information between the continuous random input $X$ and the continuous output $Y$, whereas $\beta$ is the minimum energy transfer requirement. The optimisation problem (9) aims for finding a test information source $X^{*}$, whose cumulative distribution function (CDF) $F_{X^{*}}(x)$ is capable of maximising the average mutual information $I(X ; Y)$, while satisfying the minimum energy requirement $\beta$. Upon substituting the optimal $\mathrm{CDF}_{X_{X^{*}}}(x)$ into (9), we may derive the rate-energy function of the CICO-MC. Assuming that the input message falls into the region $[-\alpha, \alpha]$, the rate-energy function can be defined as $C(\alpha, \beta)$.

Let us now study the AWGN channel as an example of the CICO-MC. In the AWGN channel, the Gaussian distributed noise $N$ has a mean of zero and a variance of $\sigma_{N}^{2}$. The output of the AWGN channel can be expressed as $Y=X+N$, whose average energy can be formulated as $\mathbb{E}[g(y)]=\mathbb{E}\left[Y^{2}\right]=\mu_{x}^{2}+$

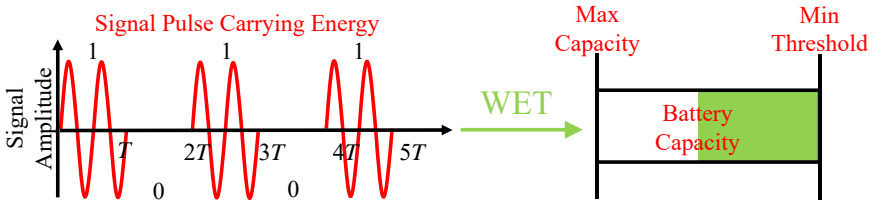

Fig. 9. On-off keying modulated RF signals for WET

$\sigma_{x}^{2}+\sigma_{N}^{2}$, where $\mu_{x}$ is the mean of the random input $X$ and $\sigma_{x}^{2}$ is its variance.

In the case of the AWGN channel, the optimisation problem (9) can be expressed as

$$
\begin{aligned}
& \text { Objective: } \max _{F_{X}(x)} H_{c}(Y)-H_{c}(N)=H_{c}(Y)-\frac{1}{2} \log 2 \pi e \sigma_{N}^{2} \\
& \text { Subject to: } \mathbb{E}[g(y)]=\mu_{x}^{2}+\sigma_{x}^{2}+\sigma_{N}^{2} \geq \beta .
\end{aligned}
$$

Naturally, we have to maximise the continuous entropy $H_{c}(Y)$ for the random output $Y$. Since the average power $P_{Y}=\mathbb{E}[g(y)]$ of the output message is constrained both by the average power of the input message as well as by the power of the noise, $H_{c}(Y)$ becomes maximum, when $Y$ follows a Gaussian distribution having a zero mean and a variance of $\sigma_{y}^{2}=P_{Y}$. Since the noise $N$ is Gaussian distributed, the random input message $X$ has to be a Gaussian distributed random variable having a zero mean as well. According to the constraint (10a), the variance of $X$ should obey $\sigma_{x}^{2} \geq\left(\beta-\sigma_{N}^{2}\right)$. Since the variance of the output message $Y$ is $\sigma_{y}^{2}=\sigma_{x}^{2}+\sigma_{N}^{2}$, the rate-energy function of the AWGN channel can thus be expressed as

$$
\begin{aligned}
C_{A W G N}(\beta) & =H_{c, \max }(Y)-\frac{1}{2} \log 2 \pi e \sigma_{N}^{2} \\
& =\frac{1}{2} \log 2 \pi e \sigma_{y}^{2}-\frac{1}{2} \log 2 \pi e \sigma_{N}^{2} \\
& =\frac{1}{2} \log \frac{\sigma_{x}^{2}+\sigma_{N}^{2}}{\sigma_{N}^{2}}=\frac{1}{2} \log \frac{\beta}{\sigma_{N}^{2}} .
\end{aligned}
$$

\section{Joint Energy and Information Coding}

Channel coding, also known as the forward error correction coding incorporates redundant bits into the original information stream at the transmitter side in order to correct the errors imposed by the hostile wireless channel. With the aid of the redundant bits, the receiver becomes capable of automatically correcting the erroneous bits, hence recovering the original information bits. As stated by information theory, channel coding allows us to communicate at a vanishingly low error probability at near-capacity rates. In the scenario of IDEN, every bit carries energy. As a result, redundant bits are not only capable of correcting errors, but they are also capable of delivering energy to the information destinations. Moreover, the information sources typically do not generate symbols with equal probabilities. For instance, according to the statistics, the letter "e" appears in modern English with a probability of $12.7 \%$. By exploiting the specific probability of the information symbols, we are capable of designing more efficient joint source and channel coding schemes for achieving efficient information transmission as well as energy delivery. 
Different symbols transmitted by the transmitter may carry different amount of energy, when the amplitude of the RF signal is exploited for conveying modulated information, such as amplitude shift keying (ASK) and quadrature amplitude modulation (QAM). Let us take the simplest on-off keying (OOK) modulation scheme as an example. In the OOK scheme, the information bit ' 1 ' is represented by the presence of the carrier waveform $A \sin (2 \pi f t)$, where $A$ is the amplitude and $f$ is the frequency of the carrier waveform. As a result, when the bit ' 1 ' is transmitted, the amount of energy carried by its modulated waveform should be expressed as $g(1)=\int_{0}^{T} A^{2} \sin ^{2}(2 \pi f t) d t=\frac{A^{2}}{2}-\frac{A^{2}}{8 \pi f} \sin (4 \pi f T)$, where $T$ is the symbol duration. If $T$ is the period of the carrier waveform, we have $g(1)=\frac{A^{2}}{2}$. Furthermore, in the OOK modulation scheme, the information bit ' 0 ' is represented by the absence of the carrier waveform. Therefore, the amount of energy carried by the bit ' 0 ' is $g(0)=0$. The OOK modulated binary symbols 0 and 1 are exemplified by Fig.9. Similar analysis can be exploited for evaluating the energy carried by a specific symbol, when other ASK and QAM modulation schemes are invoked. The attenuation of the hostile channel may result in the distortion of information transmission and the uncertainty of energy transfer.

As a result, the arrival of the wireless energy at the battery of the receiver can be modelled by a stochastic process, rather than a deterministic one. Moreover, the depletion of energy from the battery may also be modelled by a stochastic process, which usually depends on the specific action of the IDEN terminal at the receiver side. If the IDEN receiver is a sensor, the energy in the battery may be dissipated by carrying out sensing tasks or by uploading sensing results. If the IDEN receiver is a mobile device, the energy in the battery may be dissipated also by supporting its computation and communication tasks. Therefore, the energy stored in the battery at the receiver side can be modelled by a dynamic queueing process, as shown in the stylised Fig.9, whose statistical properties can be evaluated by mathematical tools, such as the classic Markov queueing theory [109], large deviation theory [110] etc. In order to support the routine operation of the IDEN terminal, on one hand, we have to set up a minimum energy threshold and ensure that the energy stored in the battery is always above this threshold. In a mathematical language, the terminal may tolerate that the underflow probability $p_{u f}$ defining the likelihood of the energy stored in the battery being lower than the minimum threshold should be higher than a predefined value of $P_{u f}$. On the other hand, in order to avoid the energy waste, when the energy stored in the battery approaches its capacity, the energy transfer should be reduced in order to avoid the waste of energy and overcharging. In a mathematical term, the terminal may tolerate that the probability $p_{\text {of }}$ of the energy supply overflowing the battery capacity should be lower than a predefined value of $P_{o f}$.

Therefore, we have to design an appropriate coding scheme subject to these battery-status related WET requirements in order to maximise the information transmission rate. This optimisation problem can be formulated as

$$
\begin{aligned}
& \text { Objective: } \max _{p(x), x \in X} I(X ; Y), \\
& \text { Subject to: } p_{o f} \leq P_{o f}, p_{u f} \leq P_{u f},
\end{aligned}
$$

where $X$ and $Y$ represents the random input message and output message, respectively, $p(x)$ is the probability of a specific codeword $x$ in the codebook $X$ being invoked, while $I(X ; Y)$ is the average mutual information between the input message $X$ and the output message $Y$.

There are some initial attempts in the literature on joint energy and information coding design. Babero et al. [111] studied the family of constrained codes for power transfer, modulation and error control in the context of the reader-totag channels in near-field passive radio frequency identification (RFID) systems. In their coding scheme, a block of information consists of the power content bits and the information content bits in order to achieve integrated data and energy transfer. However, the coding scheme of [111] was designed for a channel between a pair of inductive coupling coils, which is incapable of addressing the practical issues of the RF signal based IDEN. In [112], the constrained run-length limited codes were invoked for IDEN by replacing conventional unconstrained capacity achieving codes. According to the simulation results of Fouladgar et al. [112], the codeword structure enabling the integrated data and energy transfer can be adjusted more readily to the receiver's energy consumption pattern than the family of classic unstructured codes. Tandon et al. [113] proposed constant sub-block composition codes for the real-time simultaneous information and energy transfer. In their scheme, all sub-blocks have the same fixed composition of the energy bits and information bits, which is carefully designed for striking a trade-off between the information rate and the amount of energy transferred. Moreover, in [114], the same group of authors provided a necessary and sufficient condition for the sub-block length of the constant sub-block composition code in order to avoid any WET outage. Then they proposed a generalised sub-block energy-constrained codes by ensuring that sufficient energy is transferred within every sub-block duration [115]. Specifically, they presented a tight lower bound for the constant sub-block composition code sequences on the average energy per symbol within a sliding time window.

\section{HaRdWARe Implementation For RF Based WET}

Due to the channel's attenuation, RF-signal based WET suffers from a low energy delivery efficiency. For example, the power harvested from the received RF signal may be as low as $1 \mathrm{~mW}$, when the signal is transmitted at a power of 1 Watt. As a result, the meagre energy harvested from the RF signals cannot satisfy the demand of industrial wireless charging and it cannot readily support communications either. Generally, the degradation of WET efficiency is imposed by the following stages:

- Energy loss of DC-RF converters. At the transmitters, the information bits (or dummy information bits dedicated to WET) are modulated and shifted to the pass-band for traversing a long distance towards the receivers. The DC 
energy is converted to the amplitude of RF signals. During this process, non-negligible energy is consumed by the DC-RF converters, which imposes a DC-RF energy loss.

- Energy loss of channel attenuation. During their propagation, the RF signals are significantly attenuated by the wireless channels. When only considering the path-loss effects, the power of RF signals may be attenuated by at least $20 \mathrm{~dB}$ after they travel $10 \mathrm{~m}$ from transmitters, upon assuming line-of-sight (LoS) propagation. Furthermore, the channel attenuation becomes more serious, when both shadowing and multipath fading are taken into account.

- Energy loss of RF-DC converters. Due to its AC nature, the energy gleaned from the RF signals cannot drive substantial electronic loads or charge batteries. RF-DC converters have to be invoked for converting the RF energy into DC. However, during this energy conversion process, the RF-DC converters impose an inevitable energy loss, which reduces the amount of energy arriving at the loads.

- Energy loss of battery charging. After RF-DC converters, the resultant DC is often insufficient for driving any electronic loads or for charging batteries. Therefore, multistage amplifiers have to be invoked for further boosting the DC so as to obtain a sufficient voltage for battery charging. The electronic components of voltage amplifiers also result in non-negligible energy loss.

In this section, we will investigate solutions to the abovementioned energy loss issues in a complete WET chain.

\section{A. DC-RF Converter Design}

In order to achieve a high efficiency of the RF signal based WET, we have to carefully design the DC-RF converter of the transmitters in order to reduce energy loss during the DCRF conversion process. Since the DC-RF converter dissipates lower energy than any other component of the complete WET chain, we may expect at least $80 \%$ conversion efficiency after a careful DC-RF converter design.

A classic solid-state DC-RF converter is exemplified in Fig.10, which adopts an all solid-state ${ }^{2}$ distributed energy conversion technique. There are five major components in this DC-RF converter, namely a frequency source, a two-stage driving amplifier, a power dividing network, an amplitudephase control network and an inverse F-type Doherty power amplifier (IFT-DPA). The antenna array portrayed in Fig.10 is exploited for the combination and emission of the RF signals. The frequency source generates a low-power RF signal at a frequency of $2.45 \mathrm{GHz}$ or $5.8 \mathrm{GHz}$. After the gain amplification, a medium-power RF signal is then transferred to the power dividing network having 16 sub-patches in total. After being processed by the amplitude-phase control network for each patch, the power of the RF signal is amplified again by the IFT-DPA and the output high-power signals are finally combined and emitted by the antenna array. However, the converter of Fig.10 fails to substantially improve the DC-RF

\footnotetext{
${ }^{2}$ Solid-state electronics are those circuits or devices built entirely from solid materials and in which the electrons, or other charge carriers, are confined entirely within the solid material.
}

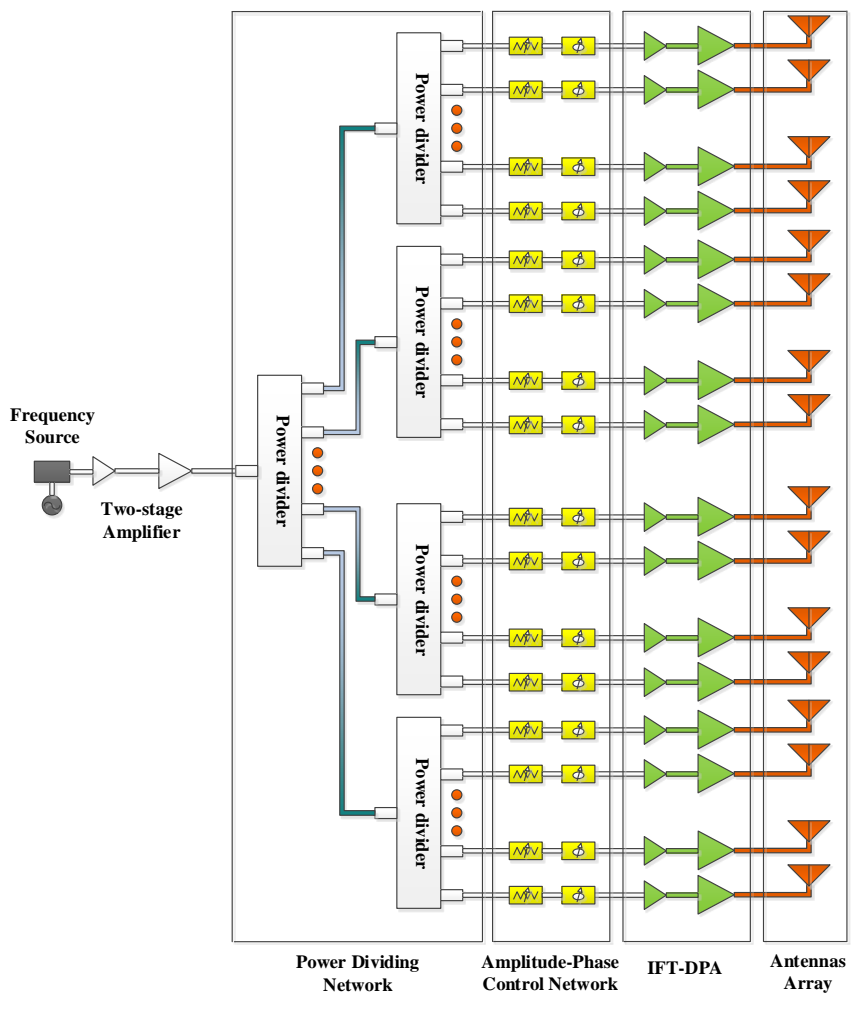

Fig. 10. Distributed all-solid-state DC-RF converter

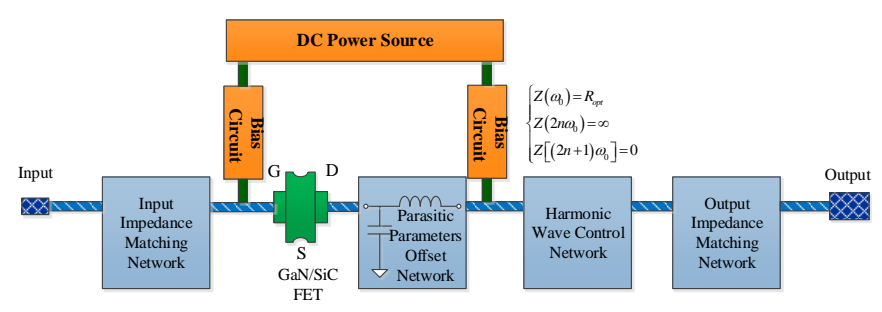

Fig. 11. The structure of an IFT-DPA

conversion efficiency, since it requires a high DC voltage for DC-RF conversion and a complete circuit has to be invoked. Nonetheless, the all solid-state distributed DC-RF converter of Fig.10 has some significant advantages, such as low energy consumption, convenient controllability, high reliability and stability, which makes it still a competitive candidate for implementing DC-RF conversion.

The power amplifier is essential in the DC-RF converter design. The power-added efficiency (PAE) of the amplifier may further increase its output power. Therefore, the power consumption of DC-RF converters can be remarkably reduced. As illustrated in Fig.10, IFT-DPA plays an important role in DC-RF conversion, in which a Doherty power amplifier is invoked.

The structure of the IFT-DPA is exemplified in Fig.11. The signal having a medium power level is firstly transmitted to the input impedance matching network and then to the gallium nitride $(\mathrm{GaN})$ or silicon carbide $(\mathrm{SiC})$ field effect transistor (FET) amplifier with bias circuits and parasitic parameters 
TABLE III

DC-RF EFFICIENCY OF THE IFT-DPA

\begin{tabular}{|c|c|c|c|c|c|}
\hline & $\mathrm{p}=1$ & $\mathrm{p}=3$ & $\mathrm{p}=5$ & $\ldots$ & $\mathrm{p}=\infty$ \\
\hline $\mathrm{q}=1$ & $50.0 \%$ & $57.7 \%$ & $60.3 \%$ & $\ldots$ & $63.7 \%$ \\
\hline $\mathrm{q}=2$ & $70.7 \%$ & $81.7 \%$ & $85.3 \%$ & $\ldots$ & $90.0 \%$ \\
\hline $\mathrm{q}=4$ & $75.0 \%$ & $86.6 \%$ & $90.5 \%$ & $\ldots$ & $95.5 \%$ \\
\hline$\ldots$ & $\ldots$ & $\ldots$ & $\ldots$ & $\ldots$ & $\ldots$ \\
\hline $\mathrm{q}=\infty$ & $78.5 \%$ & $90.7 \%$ & $94.8 \%$ & $\ldots$ & $100 \%$ \\
\hline
\end{tabular}

offset network. The input impedance matching network allows most of the fundamental wave energy to pass but filters the high-order harmonics. The parasitic parameters offset network eliminates the parasitic components incurred by the highfrequency operations of the $\mathrm{GaN} / \mathrm{SiC}$ FET. The harmonic wave control network has a pair of states for filtering the high-order harmonics of the RF waves, namely open state for filtering the even-order harmonics and short state for filtering the oddorder harmonics. As a result, only the fundamental mode of the RF wave can be transferred to the output impedance matching network.

TABLE IV

Power-Added-Efficiency (PAE) of Different Power Amplifier

\begin{tabular}{|c|c|c|c|c|}
\hline Literature & CMOS Size & Frequency & Topology & PAE \\
\hline $\begin{array}{l}\text { Vorapipat } \\
\text { et al. [116] }\end{array}$ & $\begin{array}{r}65 \mathrm{~nm} \\
\mathrm{CMOS}\end{array}$ & $0.9 \mathrm{GHz}$ & VMD & $45 \%$ \\
\hline $\begin{array}{l}\mathrm{Hu} \\
\text { et al. [117] }\end{array}$ & $\begin{array}{r}65 \mathrm{~nm} \\
\text { CMOS }\end{array}$ & $3.5 \mathrm{GHz}$ & Doherty & $34 \%$ \\
\hline $\begin{array}{l}\text { Yoo } \\
\text { et al. [118] }\end{array}$ & $\begin{array}{r}65 \mathrm{~nm} \\
\text { CMOS }\end{array}$ & $2 \mathrm{GHz}$ & ClassG & $44 \%$ \\
\hline $\begin{array}{l}\text { Kaymaksut } \\
\text { et al. [119] }\end{array}$ & $\begin{array}{r}40 \mathrm{~nm} \\
\text { CMOS }\end{array}$ & $1.9 \mathrm{GHz}$ & Doherty & $34 \%$ \\
\hline $\begin{array}{l}\text { Ye } \\
\text { et al. }[120]\end{array}$ & $\begin{array}{r}65 \mathrm{~nm} \\
\text { CMOS }\end{array}$ & $2.2 \mathrm{GHz}$ & XFMR & $43 \%$ \\
\hline $\begin{array}{l}\text { Yuan } \\
\text { et al. [121] }\end{array}$ & $\begin{array}{r}65 \mathrm{~nm} \\
\text { CMOS }\end{array}$ & $2 \mathrm{GHz}$ & ClassG & $22 \%$ \\
\hline $\begin{array}{l}\text { Gopalrao } \\
\text { et al. }[122]\end{array}$ & $\begin{array}{l}130 \mathrm{~nm} \\
\text { CMOS }\end{array}$ & $1.5-3 \mathrm{GHz}$ & $\begin{array}{r}\text { 2-stage } \\
\text { ClassEF }\end{array}$ & $27.7 \%$ \\
\hline $\begin{array}{l}\text { Madureira } \\
\text { et al. }[123]\end{array}$ & $\begin{array}{l}\text { 130nm } \\
\text { CMOS }\end{array}$ & $2.5 \mathrm{GHz}$ & ClassEF & $49.8 \%$ \\
\hline $\begin{array}{l}\text { Mazzanti } \\
\text { et al. [124] }\end{array}$ & $\begin{array}{l}\text { 130nm } \\
\text { CMOS }\end{array}$ & $1.7 \mathrm{GHz}$ & ClassF & $60 \%$ \\
\hline $\begin{array}{l}\text { Tan } \\
\text { et al. [125] }\end{array}$ & $\begin{array}{l}130 n m \\
\text { CMOS }\end{array}$ & $2.4 \mathrm{GHz}$ & ClassE & $55 \%$ \\
\hline $\begin{array}{l}\text { Takenaka } \\
\text { et al. }[126]\end{array}$ & $\begin{array}{r}\mathrm{InGaP} / \mathrm{GaAs} \\
\mathrm{HBT}\end{array}$ & $2.5 \mathrm{GHz}$ & Doherty & $41.3 \%$ \\
\hline $\begin{array}{l}\text { Kang } \\
\text { et al. [127] }\end{array}$ & $\begin{array}{r}\text { InGaP/GaAs } \\
\text { HBT }\end{array}$ & $1.85 \mathrm{GHz}$ & Doherty & $36.3 \%$ \\
\hline $\begin{array}{l}\text { Cho } \\
\text { et al. [128] }\end{array}$ & $\begin{array}{r}\mathrm{InGaP} / \mathrm{GaAs} \\
\text { HBT }\end{array}$ & $1.9 \mathrm{GHz}$ & Doherty & $45.8 \%$ \\
\hline
\end{tabular}

According to the statistic listed in Table III, the proposed converter may achieve a near $100 \%$ DC-RF conversion efficiency, when both the key parameters $q$ and $p$ tend to infinity. Here, the parameters $q$ and $p$ represent the even and odd orders, respectively, of the harmonic wave control network as shown in Fig.11. For example, the 5-order harmonic wave control network can suppress the odd harmonics up to 5th order and the even harmonics up to 4 th order, which achieve the DCRF conversion efficiency of $90.5 \%$, as illustrated in TABLE III. Furthermore, plenty of power amplifier designs [116]-[128] in literature may achieve various PAE. The operating frequency

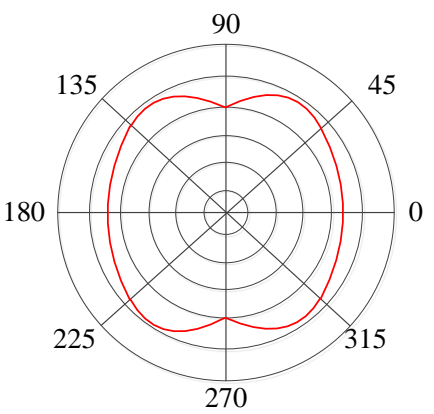

Dipole Omnidirectional

E-Plane Radiation Pattern

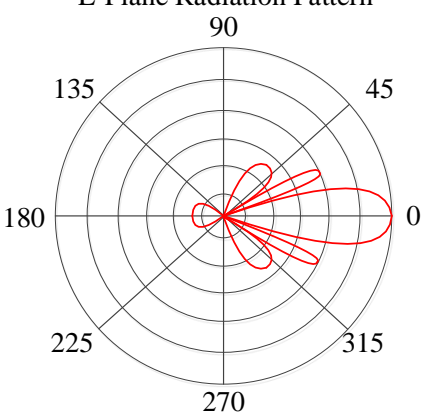

Directional E-Plane Radiation Pattern

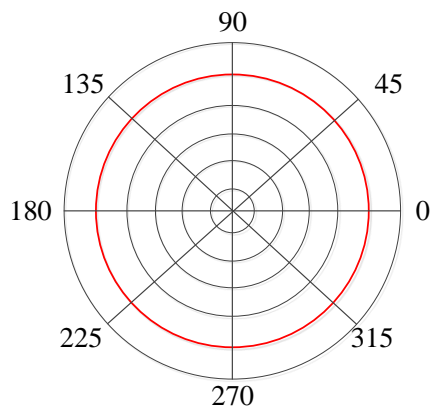

Dipole Omnidirectional H-Plane Radiation Pattern 90

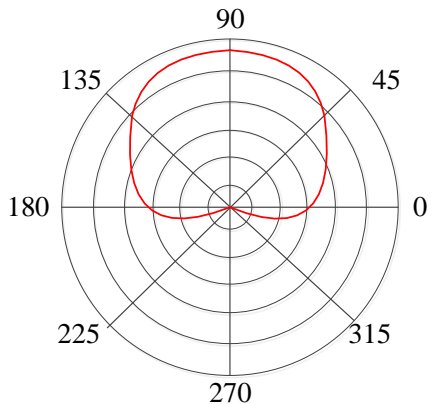

Directional H-Plane Radiation Pattern
Fig. 12. Radiation Patterns of Omnidirectional and Directional Antennas

of these power amplifiers spans from $1 \mathrm{GHz}$ to $3 \mathrm{GHz}$, while different sizes of complementary metal oxide semiconductor (CMOS) are implemented in their circuits. Specifically, $\mathrm{Hu}$ et al. [117] and Kaymaksut et al. [119] have designed the Doherty power amplifiers by invoking the different sizes of CMOS, but their PAEs both achieve 34\%. Similarly, Takenaka et al. [126], Kang et al. [127] and Cho et al. [128] have all implemented Indium gallium phosphide ( $\mathrm{InGaP}$ ) or Gallium Arsenide (GaAs) based heterojunction bipolar transistor (HBT) into their Doherty power amplifiers for the sake of further improving their PAEs. Apart from the Doherty power amplifier, some other designs may achieve a higher PAE. For example, Mazzanti et al. [124] have developed a power amplifier by invoking a $130 \mathrm{~nm}$ CMOS and a Class-F topology. This amplifier operating at a frequency of $1.7 \mathrm{GHz}$ may achieve upto $60 \%$ PAE. The PAEs of the most up-to-date power amplifier designs are summarized in Table IV. On the other hand, the magnetron [129] is capable of achieving a PAE of upto $80 \%$ in the frequency of $2.45 \mathrm{GHz}$ or of $5.8 \mathrm{GHz}$, but this is out of the scope of our treatise.

\section{B. WET over RF Channels}

A major portion of the RF signals' energy is lost during their propagation over hostile wireless channels, since the path-loss, shadowing, multipath fading and medium penetration seriously attenuate the RF signals.

1) Directional Antenna: When the RF signals traverse a long distance, the most dominant channel attenuation component is the path-loss. Furthermore, omnidirectional antennas radiate the energy in a spheral fashion, whose radius is the 
transmission distance of the RF signals. If the transmission distance is long, only a tiny portion of the energy can be collected by the receivers. As a result, directional antennas should be invoked in order to focus the energy in a certain direction, which may efficiently reduce the energy loss incurred by the long-distance propagation. The radiation patterns of the diode omnidirectional and directional antennas are portrayed in Fig.12. For the radiation pattern of a pair of the main planes of PIN diode, the E-plane ${ }^{3}$ shows ' $\infty$ ' shape pattern but the H-Plane ${ }^{4}$ exhibits the omnidirectional pattern. By contrast, the E-plane of a typical directional antenna forms a highly concentrated beam of energy at a specific direction, while its H-plane also exhibits a directional radiation pattern.

The antenna gain, which is measured in terms of $\mathrm{dBi}$, is a key parameter of characterising an antenna's capability of compensating for the channel's attenuation. This parameter represents the additional power gain attained by directional antennas over omnidirectional ones, which is formulated as $G=10 \log \left(P_{1} / P_{2}\right)$, where $P_{1}$ is the power received at a specific point when the RF signals are radiated by a directional antenna, whereas $P_{2}$ is the power received at the same point, when the RF signals are radiated by an omnidirectional antenna.

Substantial efforts [130]-[135] have been invested in the antenna design for the sake of enhancing the achievable antenna gain. Specifically, Mao et al. [130] have designed a directional antenna equipped with a coupled resonator. Their design is capable of achieving $9 \mathrm{dBi}$ antenna gain at a frequency of 5 $\mathrm{GHz}$ and it may also achieve as much as $10 \mathrm{dBi}$ antenna gain at a frequency of $6 \mathrm{GHz}$. Furthermore, Huang et al. [131] have further enhanced the antenna gain to $15 \mathrm{dBi}$ and $18 \mathrm{dBi}$ at a frequency of $5 \mathrm{GHz}$ and that of $4.5 \mathrm{GHz}$, respectively, by exploiting the so-called Dual Fed Stacked Path Antenna Array. The latest contributions concerning antenna design are summarised in TABLE V.

However, a typical high directional antenna fails to reduce the energy loss incurred by beam diffraction. As a result, the Gaussian beam as well as the non-diffracted electromagnetic waves such as Bessel beams and Bessel-Gaussian beams [136] can be exploited for the sake of further alleviating the adverse effect of beam diffraction. Relying on their non-diffracting nature, the Bessel-Gaussian beams are capable of efficiently reducing the energy dissipation and hence improve the WET efficiency over wireless channels.

2) Energy Reception Efficiency: When the RF signals arrive at a receiver, not all of their energy can be gleaned by the receiver, subject to the materials and structures adopted by the energy harvester, which may result in unavoidable energy leakage. As a result, artificial electromagnetic structures as well as super-surfaces can be adopted for the sake of efficiently collecting energy carried by the RF signals [137]. Hence, different artificial electromagnetic cells are capable of receiving

\footnotetext{
${ }^{3}$ For a linearly-polarized antenna, the E-plane contains the electric field vector (sometimes called the E-aperture) and the direction of maximum radiation.

${ }^{4}$ For the same linearly-polarized antenna, the H-plane contains the magnetic field vector (sometimes called the $\mathrm{H}$-aperture) and the direction of maximum radiation.
}

TABLE V

SUMMARISATION OF LATEST ANTENNA DESIGN

\begin{tabular}{|l|r|r|r|}
\hline Literature & Antenna Design & Frequency & Gain \\
\hline \hline $\begin{array}{l}\text { Mao } \\
\text { et al. }[130]\end{array}$ & $\begin{array}{r}\text { Antenna with Coupled } \\
\text { Resonators }\end{array}$ & $5.0 \mathrm{GHz}$ & $9 \mathrm{dBi}$ \\
\hline $\begin{array}{l}\text { Mao } \\
\text { et al. }[130]\end{array}$ & $\begin{array}{r}\text { Antenna with Coupled } \\
\text { Resonators }\end{array}$ & $6.0 \mathrm{GHz}$ & $10 \mathrm{dBi}$ \\
\hline $\begin{array}{l}\text { Huang } \\
\text { et al. }[131]\end{array}$ & $\begin{array}{r}\text { Dual Fed Stacked Patch } \\
\text { Antenna Array }\end{array}$ & $4.5 \mathrm{GHz}$ & $18 \mathrm{dBi}$ \\
\hline $\begin{array}{l}\text { Huang } \\
\text { et al. }[131]\end{array}$ & $\begin{array}{r}\text { Dual Fed Stacked Patch } \\
\text { Antenna Array }\end{array}$ & $5 \mathrm{GHz}$ & $15 \mathrm{dBi}$ \\
\hline $\begin{array}{l}\text { Wang } \\
\text { et al. }[132]\end{array}$ & $\begin{array}{r}\text { Transmission Polarisation } \\
\text { Meta-Surface Antenna }\end{array}$ & $10.6 \mathrm{GHz}$ & $14 \mathrm{dBi}$ \\
\hline $\begin{array}{l}\text { Jiang } \\
\text { et al. }[133]\end{array}$ & Holographic Antenna & $35 \mathrm{GHz}$ & $18 \mathrm{dBi}$ \\
\hline $\begin{array}{l}\text { Su Antenna } \\
\text { et al. }[134]\end{array}$ & $\begin{array}{r}\text { Surface-Wave Holographic } \\
\text { Ang }\end{array}$ & $20 \mathrm{GHz}$ & $16.5 \mathrm{dBi}$ \\
\hline $\begin{array}{l}\text { Shafer } \\
\text { et al. } \text { [135] }\end{array}$ & $\begin{array}{r}\text { Planar Frequency Scanning } \\
\text { Holographic Antenna }\end{array}$ & $240 \mathrm{GHz}$ & $18 \mathrm{dBi}$ \\
\hline
\end{tabular}

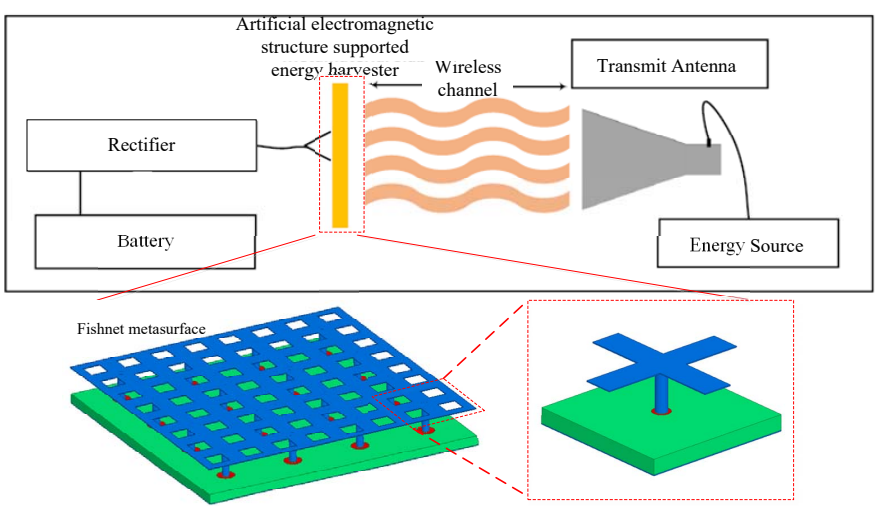

Fig. 13. Artificial electromagnetic structure supported energy receiver

the energy carried by RF signals having different bandwidths and frequencies, which have been reported in [138]-[141] in the past few years. The typical artificial electromagnetic structure based energy receiver is illustrated in Fig.13. The RF energy radiated by the transmit antenna reaches the surface of the artificial electromagnetic structure and it can be absorbed by the meta-material cells due to the high-efficiency absorbing mechanism of the meta-material. The absorbed energy is then stored in the resistance or rectified by the diode. Compared to the classic rectennas, the meta-material/meta-surface based energy harvesters have the following advantages: low cost, low weight, easy to fabricate and assemble, high energy harvesting efficiency and compatibility with any polarizations and angles of the received RF signals. Furthermore, the operational power range of the meta-material/meta-surface based energy harvester should also be considered in the future design of the WET system.

\section{RF-DC Converter Design}

The typical model of an RF-DC converter is depicted in Fig.14. The RF signals radiated by the transmitters are firstly captured by the receivers' antennas, which are modelled by an RF voltage generator, plus its internal resistance and impedance. Then, the captured signals flow into a matching network in order to match the impedance of the antennas 


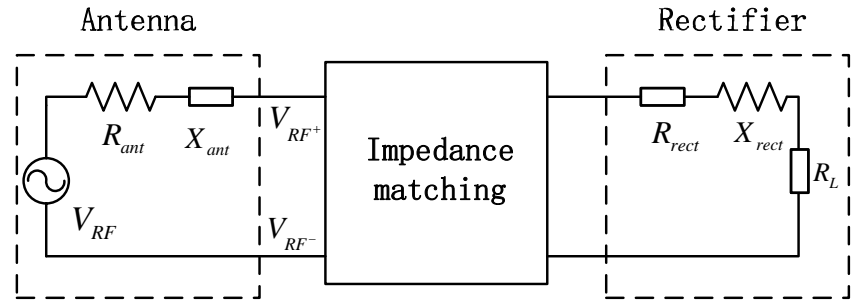

Fig. 14. Architecture of the RF-DC converter

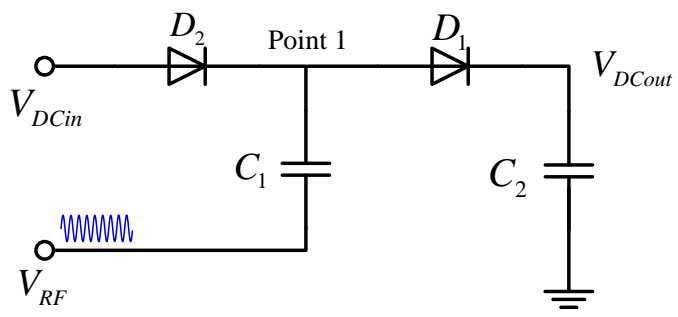

Fig. 15. Voltage doubler rectifier

and that of the rectifiers and the loads. Accurate impedance matching ensures the maximum energy transfer from the antennas to the loads. The rectifier converts the RF signals into the DC, which is then exploited either for driving electronic loads or for charging the batteries.

1) Impedance matching: The impedance mismatch between the antennas and the rectifiers may seriously degrade the RFDC conversion efficiency. In order to achieve a high RF-DC conversion efficiency, a impedance matching network can be operated as a resonator having a quality factor of $Q=f_{c} / \Delta f$, where $f_{c}$ represents the central frequency of the RF-DC conversion, while $\Delta f$ represents the corresponding conversion bandwidth. However, according to its definition, given a specific $f_{c}$, a high $Q$ is associated with a low conversion bandwidth $\Delta f$ [142]. As a counter-measure, the resonator's capacitive and inductive have to be carefully designed for preventing the high-Q resonator from limiting the RF-DC conversion bandwidth. Explicitly, in the case of a series of matched LC resonators, the reactive components are chosen to be the complex conjugates of each other, while the resistive components are also matched. The factor $Q_{L C}$ can be further expressed as $Q_{L C}=\left(\omega L_{L C}\right) /\left(2 R_{L C}\right)=X_{L C} /\left(2 R_{L C}\right)$ [143], where $X_{L C}$ and $R_{L C}$ represents the reactive component and the resistive component of the LC resonator, respectively.

2) Rectifier Circuits: The rectifiers are invoked for converting $\mathrm{RF}$ (or $\mathrm{AC}$ ) signals to $\mathrm{DC}$ signals. There are three different types of voltage doubler rectifiers for realising RF-DC conversion, namely the basic voltage doubler rectifier [144], the floating-gate based voltage doubler rectifiers [145] and the multi-stage rectifier [146].

A voltage doubler rectifier [144] is capable of rectifying the full-wave RF signals. A typical circuit topology of the voltage doubler rectifier is illustrated in Fig.15, which relies on a voltage clamp and a peak rectifier. An operating cycle of the voltage doubler rectifier consists of a pair of consecutive phases:
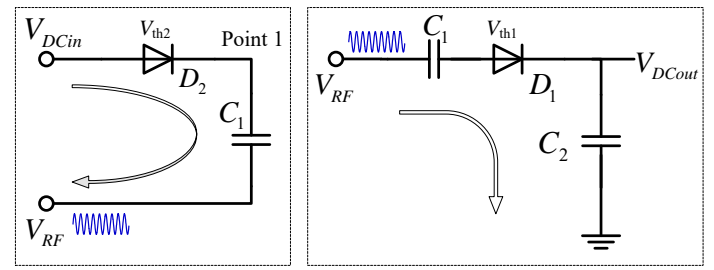

Fig. 16. Two phases of the voltage doubler rectifier

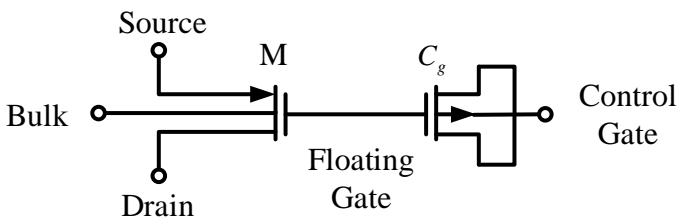

Fig. 17. The topology of the floating-gate

- During the negative phase of the input RF signal, as portrayed in Fig.16(a), the current flows through the diode $D_{2}$ while the diode $D_{1}$ is in the outage. The voltage across the diode $D_{2}$ will stay constant around its threshold voltage and the voltage at "Point 1" of Fig.15 is charged to $V_{t h 2}$. At the negative peak, the voltage across capacitor $C_{1}$ is $V_{\text {out }}=V_{R F}-V_{\text {th } 2}$.

- During the positive phase of the input RF signal, as portrayed in Fig.16(b), the current flows through the diode $D_{1}$, while the diode $D_{2}$ is in the outage. Since the voltage of the capacitor $C_{1}$ has already been charged to $\left(V_{R F}-V_{t h 2}\right)$ during the negative phase of the input RF signal and remains unchanged, the voltage across the diode $D_{2}$ is then expressed as $\left(2 V_{R F}-V_{t h 2}\right)$ during the current phase. Since the diode $D_{1}$ conducts the current in order to charge the capacitor $C_{2}$, the output voltage is $V_{\text {out }}=2 V_{R F}-V_{\text {th } 1}-V_{\text {th } 2}$.

The topology of the floating gate based transistor is designed for the sake of reducing the activation threshold of the diode in the voltage doubler rectifier circuit. When the charge is injected into the floating gate based transistor, whose topology is illustrated in Fig.17, the charge remains in the gate of oxide provided by the oxide layer due to the high impedance.

The typical floating gate of Fig.17 consists of two types of positive metal oxide semiconductor (PMOS) transistors, namely the M-type transistor and the Cg-type transistor. A Cg-type PMOS transistor is capacitively connected to the gate of the M-type PMOS transistor, while the Bulk port of the Mtype transistor and the control gate of the Cg-type transistor are both connected to the rear of the M-type transistor. When the charges are injected into the capacitor, a bias-voltage is stimulated between the source port and the control gate. This gate-source bias voltage effectively reduces the activation threshold voltage of the floating gate, which may act as an equivalent diode in the rectifier. As a result, replacing the classic diode with the floating gate is capable of substantially reducing the energy consumption of the rectifier so as to improve the efficiency of the RF-DC conversion.

The architecture of the floating-gate based rectifier [145] is similar to that of the voltage doubler based rectifier. The diodes 


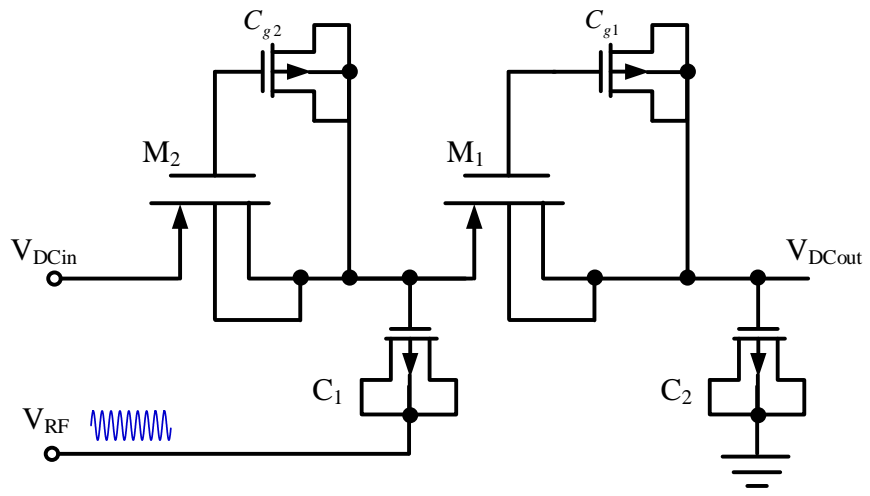

Fig. 18. Floating-gate rectifier circuit

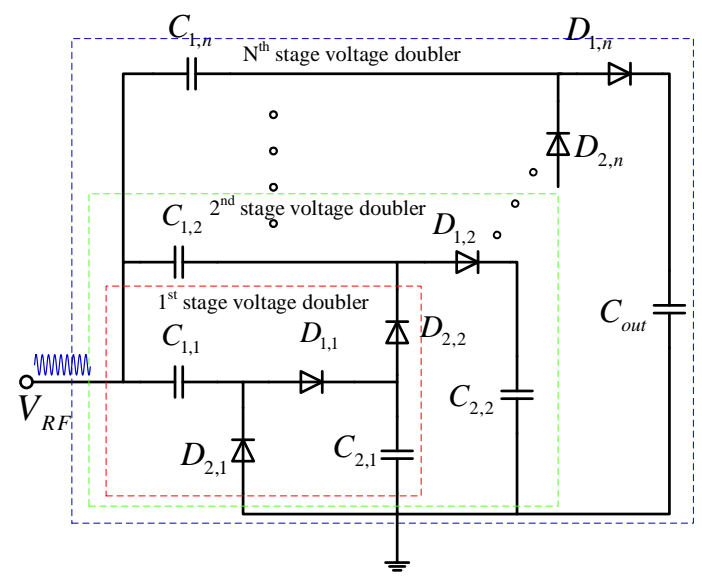

Fig. 19. Multi-stage voltage doubler based rectifier

$D_{1}$ and $D_{2}$ can be replaced by the floating gates transistors, as illustrated in Fig.18. The gate oxide is a powerful insulator, which prevents the charges from leaking off the floating gate. As a result, this rectifier is capable of efficiently increasing the output voltage and thus increasing the RF-DC conversion efficiency.

A number of the voltage doubler rectifiers can be arranged in cascade for the sake of increasing the output voltage, which is known as a multi-stage voltage doubler based rectifier [147], as illustrated in Fig.19. The voltage of the input signal can thus be increased gradually after being sequentially rectified by the $N$ stages of voltage doubler. In the multi-stage voltage doubler based rectifier, each stage operates as a voltage level shifter. However, we should carefully design the number of stages in this rectifier, since too few stages may not produce sufficient output voltage, whilst too many stages may reduce the $Q$-factor of the resonator.

High RF-DC conversion efficiency can be achieved by amalgamating complementary techniques together, as summarised in Table VI.

\section{Battery Charging Circuit Design}

After the RF-DC converter, the resultant DC energy may be immediately invoked for driving electronic loads. If the receiver does not require any energy at a specific moment,

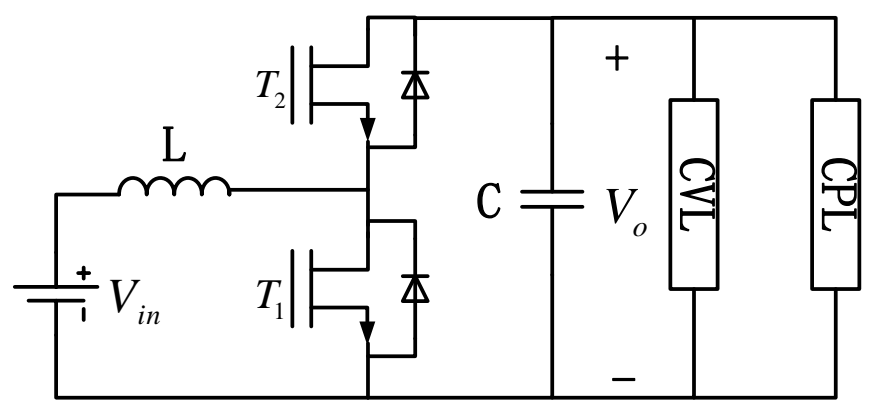

Fig. 20. A DC-DC converter for battery charging

this DC energy should be stored in the batteries for future use. However, the energy output by the RF-DC converter is always lower than that finally stored in batteries, since the battery charging circuit itself inevitably consumes a certain portion of energy. In order to improve the battery charging efficiency, both the battery charging circuits and the voltage detector circuits should be carefully designed [154].

1) Battery Charging Circuit: During the entire charging process, the battery charging circuits play the most important role, since a well-designed circuit only incurs little energy leakage and hence most of the energy can be finally stored in the batteries. Since the received energy of the RF signal is typically low, a micro-battery and a dedicated charging system have to be designed for storing and collecting this precious energy. A DC-DC converter is essential for amplifying the typically modest output DC energy of the RF-DC converter. We portray the circuit topology of a DC-DC converter in Fig.20, which is invoked for feeding stable voltage to a constant power load (CPL) and a constant voltage load (CVL). When the current flows through the transistor $T_{1}$ and the inductance $L$, the transistor $T_{2}$ is in the outage and the energy is then accumulated in the inductance $L$; When current flows through the transistor $T_{2}$ and the inductance $L$, the transistor $T_{1}$ is in the outage and the energy stored in the capacitor $C$ then drives both the constant power load and the constant voltage load. The input DC voltage $V_{i n}$ is thus boosted by the DC-DC converter to the output voltage $V_{o}$.

2) Voltage Level Detector: In order to facilitate the control and the management of the batteries, a voltage level detector is required for monitoring the battery charging state and to intelligently activate the charging or sleeping process. The detector has to ensure that the voltage of batteries should be higher than the discharging voltage threshold in order to reliably power the communication circuits.

A range of contributions on the battery charging circuit design have been summarised in TABLE VII. Specifically, the authors of [156]-[158], [160] have designed diverse TSMC ${ }^{5}$ CMOS enabled battery charging circuits relying on different integration density in order to improve the battery charging efficiency. The achievable charging efficiency they reported were $86 \%$ [156], 67.9\% [157], 87\% [158] and 89\% [160]. By

${ }^{5}$ TSMC is an anonym for Taiwan Semiconductor Manufacturing Company, Limited. 
TABLE VI

SUMMARISATION OF RF-DC CONVERTERS

\begin{tabular}{|c|c|c|c|c|}
\hline Literature & RF-DC Converter & $\begin{array}{l}\text { Operating } \\
\text { Frequency }\end{array}$ & $\begin{array}{r}\text { Input } \\
\text { Power }\end{array}$ & $\begin{array}{l}\text { Conv. } \\
\text { Effici. }\end{array}$ \\
\hline $\begin{array}{l}\text { Song } \\
\text { et al. }[148]\end{array}$ & $\begin{array}{r}\text { Six-band dual circular } \\
\text { polarisation rectenna }\end{array}$ & $\begin{array}{r}0.9 \mathrm{GHz} \\
1.86 \mathrm{GHz}\end{array}$ & $-5 \mathrm{dBm}$ & $\begin{array}{l}67 \% \\
53 \%\end{array}$ \\
\hline $\begin{array}{l}\text { Yoshida } \\
\text { et al. [149] }\end{array}$ & $\mathrm{C}$-band $\mathrm{GaN}$ diode rectifier & $5.9 \mathrm{GHz}$ & $30 \mathrm{dBm}$ & $33 \%$ \\
\hline $\begin{array}{l}\mathrm{Hu} \\
\text { et al. }[150]\end{array}$ & Closely-spaced-element rectenna & $2.45 \mathrm{GHz}$ & $-7 \mathrm{dBm}$ & $58 \%$ \\
\hline $\begin{array}{l}\text { AbdeTawab } \\
\text { et al. }[151]\end{array}$ & $\begin{array}{r}\text { Quad-band converter equipped } \\
\text { with a single antenna and a } \\
\text { single matching network }\end{array}$ & $\begin{array}{r}0.9,1.8 \\
2.1,2.4 \\
\mathrm{GHz}\end{array}$ & $\begin{array}{l}-10 \mathrm{dBm} \\
-15 \mathrm{dBm} \\
-20 \mathrm{dBm}\end{array}$ & $\begin{array}{l}86 \% \\
52 \% \\
24 \%\end{array}$ \\
\hline $\begin{array}{l}\text { Moura } \\
\text { et al. [152] }\end{array}$ & MMIC GaN Power Amplifier & $8.0 \mathrm{GHz}$ & $27 \mathrm{dBm}$ & $40 \%$ \\
\hline $\begin{array}{l}\text { Dehghani } \\
\text { et al. [153] }\end{array}$ & Tracking load circuit & N.A. & $\begin{array}{l}38 \mathrm{dBm} \\
41 \mathrm{dBm}\end{array}$ & $\begin{array}{l}55 \% \\
75 \%\end{array}$ \\
\hline
\end{tabular}

TABLE VII

Circuit Design for Battery Charging

\begin{tabular}{|c|c|c|c|}
\hline Literature & CMOS Type & $\begin{array}{r}\text { Input } \\
\text { Voltage }\end{array}$ & $\begin{array}{l}\text { Conv. } \\
\text { Effici. }\end{array}$ \\
\hline $\begin{array}{l}\text { Hyun } \\
\text { et al. [154] }\end{array}$ & UMC $0.18 \mu m$ & $3-5.5 \mathrm{~V}$ & $94.6 \%$ \\
\hline $\begin{array}{l}\text { Valle } \\
\text { et al. [155] }\end{array}$ & AMI $0.5 \mu m$ & $4.3 \mathrm{~V}$ & $89.7 \%$ \\
\hline $\begin{array}{l}\text { Pagano } \\
\text { et al. }[156]\end{array}$ & TSMC $0.18 \mu m$ & $5-10 \mathrm{~V}$ & $86 \%$ \\
\hline $\begin{array}{l}\text { Hwang } \\
\text { et al. [157] }\end{array}$ & TSMC $0.35 \mu m$ & $5 \mathrm{~V}$ & $67.9 \%$ \\
\hline $\begin{array}{l}\text { Qiu } \\
\text { et al. [158] }\end{array}$ & TSMC $0.25 \mu m$ & $0.5-2 \mathrm{~V}$ & $87 \%$ \\
\hline $\begin{array}{l}\text { Enne } \\
\text { et al. [159] }\end{array}$ & AMS H35 $0.35 \mu m$ & $0.5-2 \mathrm{~V}$ & $88 \%$ \\
\hline $\begin{array}{l}\text { Tsai } \\
\text { et al. [160] }\end{array}$ & TSMC $0.35 \mu m$ & $0.5-2 \mathrm{~V}$ & $89 \%$ \\
\hline
\end{tabular}

contrast, Valle et al. [155] have designed an $\mathrm{AMI}^{6} \mathrm{CMOS}$ enabled battery charging circuit having a size of $0.5 \mu \mathrm{m}$. The resultant battery charging efficiency was enhanced to $89.7 \%$. Moreover, a $\mathrm{UMC}^{7} \mathrm{CMOS}$ enabled battery charging circuit has been designed by Hyun et al. [154], whose size is $0.18 \mu \mathrm{m}$. The corresponding efficiency of this battery charging circuit is as high as $94.5 \%$.

\section{Transceiver Design for Physical Layer}

In order to integrate WET and WIT in IDENs, the transceiver design in the physical layer has to embrace a substantial change, which will be introduced in this section in a tutorial manner. Relying on the crucial multiple-inputmultiple-output (MIMO) system concept, the efficiency of both WET and WIT can be remarkably improved. Furthermore, we will also discuss the waveform design for the integrated WET and WIT.

\section{A. Transmitter Architecture}

As shown in Fig.21, a typical transmitter in IDENs, whose energy is supplied by the power grid, is capable of transmitting

${ }^{6} \mathrm{AMI}$ is an anonym for a PC hardware company, American Megatrends Incorporated.

${ }^{7} \mathrm{UMC}$ is an anonym for United Microelectronics Corporation

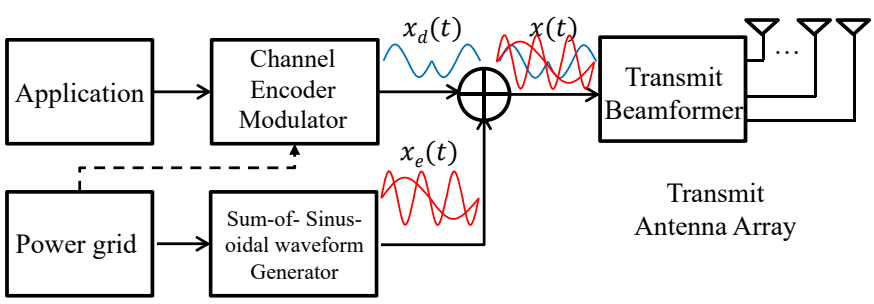

Fig. 21. Architecture of a transmitter, where the information signal $x_{d}(t)$ is exemplified by a two-bit flow modulated by BPSK, the energy signal $x_{e}(t)$ is the sum of a pair of sinusoidal waveforms and the resultant hybrid signal is $x(t)$.

both information and energy to UEs. A signal $x(t)$, which is a combination of a modulated information signal $x_{d}(t)$ and an energy signal $x_{e}(t)$, is transmitted to the receivers by multiple transmit antennas. Multiple antennas are capable of focusing the energy in a directional beam, which improves the efficiency of both WET and WIT. Since both WET and WIT can rely on a common RF signal $x(t)$, we do not have to impose additional complexity on the IDEN transmitters, while the architectural changes of the receivers required to enable integrated WET and WIT in IDEN were documented in [108], [161]-[165]. However, according to [166], [167], the superposion of multiple sinusoidals results in a high peakto-average-power-ratio (PAPR) and hence it may increase the WET efficiency relying on RF signals. This however contradicts to the design principles of WIT, where a low PAPR has to be maintained in order to achieve high WIT efficiency. As a result, the transmitter architecture has to strike a compromise for the sake of simultaneously enabling both WET and WIT services of high quality [168], [169], as portrayed in Fig.21.

\section{B. Receiver Architecture}

Again, the architecture of receivers also has to be redesigned in order to simultaneously receive data and energy from the received RF signals. In this section, we will firstly review a classic WET receiver architecture. We will then introduce the ideal architecture for IDEN receivers, which is followed by the review of the signal splitting based receiver architectures. 
1) WET Receiver Architecture: As portrayed in Figs.2225, a classic WET receiver proposed in [170] consisting of a diode, a low-pass-filter (LPF) and an energy storage unit is capable of harvesting the energy carried by the received RF signal $y_{e}(t)$. The diode converts the received RF signal $y_{e}(t)$. The LPF removes high-frequency harmonic components of the output current of the diode. The resultant DC signal $i_{D C}(t)$ can then be used for charging the energy storage unit or for driving electronic loads. Note that the output DC signal $i_{D C}(t)$ is dominated by the second-order and the fourth-order upper harmonics of the input RF signal $y_{e}(t)$ [168]. As a result, $i_{D C}(t)$ may be expressed in a non-linear form as

$$
i_{D C}(t)=k_{0}+k_{2} y_{e}^{2}(t)+k_{4} y_{e}^{4}(t)+\cdots,
$$

where the coefficients $\left\{k_{0}, k_{2}, k_{4}, \cdots\right\}$ are constants determined by the features of the diode used.

Note that Eq.(13) is pivotal in the waveform design for the sake of increasing the WET efficiency [166], [167]. The non-linearity of the RF-DC conversion has been taken into account in an IDEN system [171], where a base-station (BS) equipped with multiple antennas serves $K$ WIT receivers and $J$ WET receivers. The total energy received by WET receivers was maximised in [171] subject to the minimum signal-to-interference-plus-noise-ratio (SINR) requirement of WIT receivers. Boshkovska et al [172] have also considered the non-linear RF-DC converter in a scenario, where $K$ IDEN UEs are supported by a BS equipped with a single antenna. However, non-linear RF-DC converters may result in a less attractive performance versus complexity than their linear counterparts. A linear RF-DC converter is conceived by setting the coefficients of (13) $k_{0}=0$ and $k_{4}=0$. Furthermore, the energy finally stored in the energy storage unit is propotional to the DC signal $i_{D C}(t)$. Hence, the actual power $P_{e}$ received by the WET receiver can be expressed as [162], [163].

$$
P_{e}=\eta_{b a t} \mathbb{E}\left[i_{D C}(t)\right]=\eta_{b a t} \mathbb{E}\left[k_{2} y_{e}^{2}(t)\right]=\eta_{b a t} k_{2} P_{y_{e}}=\eta_{e} P_{y_{e}},
$$

where $\eta_{b a t} \in[0,1]$ is the battery charging efficiency, $P_{y_{e}}$ is the power of the received RF signal $y_{e}(t)$, whereas $\eta_{e}=\eta_{b a t} k_{2}$ represents the energy conversion efficiency of the WET receiver, when the linear RF-DC converter is employed.

The architecture of the WET receiver, as depicted in Figs.22-25, has been well recognised in the literature [173][177] as a practical design for supporting the RF energy harvesting function, especially when linear RF-DC converters are employed. For example, this architecture has been widely studied in WPCNs in order to realise the classic "harvest-thentransmit" protocol for supporting batteryless UEs. When this protocol is adopted, a single transmission cycle is partitioned into two portions, namely the downlink WET supported by the BS and the uplink WIT initialised by the UEs. Specifically, the energy required by the UEs' uplink WIT is supplied by the downlink WET [173]. Explicitly, a WPCN supporting $K$ batteryless UEs has been studied in [173], where both the sum-throughput and the common throughput are optimised in order to attain the maximum total uplink throughput and to ensure fairness, respectively. Liu et al. [174] have investigated the optimal beamformer design for a multiple antenna aided

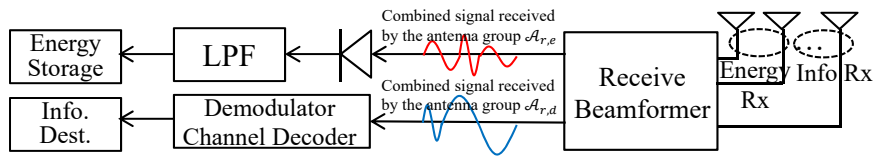

Fig. 22. Spatial splitting based receiver architecture. The combined signal received by the antenna group $\mathcal{A}_{r, e}$ is exploited for energy harvesting, while that received by the antenna group $\mathcal{A}_{r, d}$ is exploited for information decoding.

downlink WET and the SINR maximisation of the UEs' uplink transmissions. Furthermore, Ju et al. of [175] has introduced full-duplex technique into WPCNs, in which a BS may transmit energy in the downlink and receive data in the uplink at the same time. The concept of a large-scale WPCN has been proposed by Che et al. [176], while a MIMO aided cognitive WPCN supporting multiple UEs has been investigated by Kim et al. [177].

In the rest of Section V-B, different architectures conceived for supporting the integrated WET and WIT will be introduced.

2) Ideal Receiver Architecture: Varshney [108] and Grover [161] investigated a receiver architecture for integrated WET and WIT, which is capable of receiving energy and simultaneously decoding information from the same received RF signals, as detailed in Section III from an theoretical perspective. However, this ideal architecture cannot be practically realised.Hence, practical receiver relying on time switching [162] and power splitting [163] were designed. Nonetheless, this ideal receiver architecture is capable of shedding light on the performance limits of integrated WET and WIT. For example, based on the above-mentioned ideal receiver architecture, Zhou [162] and Xiang [165] have found the upper bound of rate-energy region, which characterises the fundamental tradeoff between WET and WIT.

3) Spatial Splitting Based Receiver: In the spatial splitting (SS) based receiver architecture, as portrayed in Fig.22, the signal is split by allocating different number of antennas at the receiver end for energy or information reception. Let us assume that $N_{r}$ antennas are used at the receiver. Given the splitting factor $\rho$, we may select a set $\mathcal{A}_{r, e}=\left\{A_{r, i} \mid 1 \leq i \leq\right.$ $\left.\left\lfloor\rho N_{r}\right\rfloor\right\}$ of antennas for energy reception, while the rest are adopted for information reception, which form another set $\mathcal{A}_{r, d}=\left\{A_{r, j} \mid 1 \leq j \leq N_{r}-\left\lfloor\rho N_{r}\right\rfloor\right\}$. Furthermore, if we have $N_{t}$ transmit antennas, say $\mathcal{A}_{t}=\left\{A_{t, n} \mid 1 \leq n \leq N_{t}\right\}$, at the transmitter side, then we have $N_{t} \times\left\lfloor\rho N_{r}\right\rfloor$ MIMO channels for energy delivery and $N_{t} \times\left(N_{r}-\left\lfloor\rho N_{r}\right\rfloor\right)$ MIMO channels for information transmission. Let us assume that the signal $y_{A_{r, i}}$ is received by the receive antenna $A_{r, i}$, which is expressed as

$$
y_{A_{r, i}}=\sum_{A_{t, j} \in \mathcal{A}_{t}} h_{A_{t, j}, A_{r, i}} x_{A_{t, j}}+I_{A_{r, i}}+z_{0, A_{r, i}}+z_{c o v, A_{r, i}},
$$

where $x_{A_{t, j}}$ is the information symbol transmitted by the antenna $A_{t, j}, h_{A_{t, j}, A_{t, i}}$ is the channel coefficient between the transmit antenna $A_{t, j}$ and the receive antenna $A_{r, i}, I_{A_{r, i}}$ is the total interference imposed on the receive antenna $A_{r, i}$, $z_{0, A_{r, i}}$ is the AWGN having a power of $\sigma_{0}^{2}$ imposed on the receive antenna $A_{r, i}$ and finally, $z_{c o v, A_{r, i}}$ is the additional AWGN 
introduced by the RF-band to baseband converter having a power of $\sigma_{c o v}^{2}$. Note furthermore that, the transmit power of the transmit antenna $A_{t, j}$ is denoted as $P_{t, A_{t, j}}=\left|x_{A_{t, j}}\right|^{2}$ and we can collect the transmit power of all the transmit antennas into a column vector $\mathbf{P}_{t}=\left(P_{t, A_{t, 1}}, \cdots, P_{t, A_{t, N_{t}}}\right)^{T}$.

As for the energy reception, both the interference $I_{A_{r, i}}$ and the noise $z_{A_{r, i}}$ are useful energy sources, hence the total RF power collected by the receiver should be expressed as

$$
\begin{aligned}
& P_{e}^{S S}\left(\mathcal{A}_{r, e}, \mathbf{P}_{t}\right) \\
& =\eta_{e} \sum_{A_{r, i} \in \mathcal{A}_{r, e}}\left(\left|\sum_{A_{t, j} \in \mathcal{A}_{t}} h_{A_{t, j}, A_{t, i}} x_{A_{t, j}}\right|^{2}+\left|I_{A_{r, i}}\right|^{2}+\sigma_{0, A_{r, i}}^{2}\right),
\end{aligned}
$$

where $\eta_{e}$ is the constant efficiency of the WET receiver's energy conversion. Since $\left|x_{A_{t, j}}\right|^{2}=P_{t, A_{t, j}}, P_{e, t o t a l}^{S S}\left(\mathcal{A}_{r, e}, \mathbf{P}_{t}\right)$ is a function with respect to the transmit power vector $\mathbf{P}_{t}$.

By carrying out the singular-value-decomposition (SVD) of the $N_{t} \times\left(N_{r}-\left\lfloor\rho N_{r}\right\rfloor\right)$-element MIMO channel $\mathbf{H}_{I}$, we have $\mathbf{H}_{I}=\mathbf{U}_{I} \boldsymbol{\Lambda}_{I} \mathbf{V}_{I}^{\dagger}$, where $\boldsymbol{\Lambda}_{I}$ is the diagonal matrix of the singular values of $\mathbf{H}_{I}$ with $\lambda_{i}$ on the $i$-th diagonal. Moreover, $\mathbf{U}_{I}$ and $\mathbf{V}_{I}$ are the corresponding unitary matrix, where $\mathbf{U}_{I}^{\dagger}$ and $\mathbf{V}_{I}^{\dagger}$ are the conjugate transposes of $\mathbf{U}_{I}$ and $\mathbf{V}_{I}$, respectively. Therefore, the original MIMO channel $\mathbf{H}_{I}$ is decomposed into $\operatorname{rank}\left(\mathbf{H}_{I}\right)$ independent and parallel eigen-channels. Assuming that the transmitter has perfect channel state information (CSI), the achievable information rate is expressed as

$$
R^{S S}\left(\mathcal{A}_{r, I}, \mathbf{P}_{s v d}\right)=\sum_{i=1}^{\operatorname{rank}\left(\mathbf{H}_{l}\right)} \log _{2}\left(1+\frac{\lambda_{i}^{2} P_{s v d, i}}{\left|I_{s v d, i}\right|^{2}+\sigma_{0}^{2}+\sigma_{c o v}^{2}}\right),
$$

where $P_{s v d, i}$ is the transmit power on the $i$-th eigen-channel and we have the column vector $\mathbf{P}_{s v d}=\left(P_{s v d, i}, \cdots, P_{s v d, \operatorname{rank}\left(\mathbf{H}_{I}\right)}\right)^{T}$, whereas $\left|I_{s v d, i}\right|^{2}$ is the interference power on the $i$-th eigenchannel and $\mathbf{I}_{s v d, i}=\mathbf{U}_{I}^{\dagger} \mathbf{I}_{\mathcal{A}_{r, I}}$. Furthermore, after the SVD, we have $\mathbf{P}_{t}=\left|\mathbf{V}_{I}^{\dagger}\right|^{2} \mathbf{P}_{s v d}$. Again, the additional noise power $\sigma_{c o v}^{2}$ on each eigen-channel is introduced by the RF-band to baseband converter.

In [178], by invoking an antenna selection scheme and aligning interference at the reduced signal subspace, Koo et al. partitioned the received signal into two orthogonal spaces in order to achieve simultaneous information and energy transfer. Zhao et al. [179] formulated the joint optimisation problem of antenna selection and transmit covariance matrix design in order to achieve the maximum information rate subject to the specific energy harvesting constraint encountered.

Apart from antenna selection, dual-polarised antennas can also be invoked for splitting RF signals in the spatial domain, which are capable of providing polarisation diversity, whilst alleviating the antennas' space limitation. More explicitly, two orthogonal polarisations can be created, which potentially halves the number of antennas [180]. With the aid of dualpolarised antennas adopted for integrated WET and WIT, the data signals and energy signals can be transmitted separately and they can be received independently with the aid of vertical and horizontal polarisation. Hence, the dual-polarised antenna aided integrated WET and WIT is worth further investigation due to its low-complexity and promising performance.

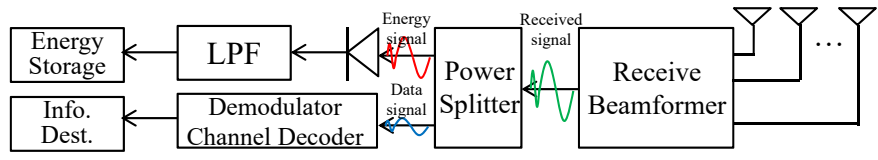

Fig. 23. Power splitting based receiver architecture. The received signal is split in the power domain. The energy signal is exploited for energy harvesting, while the data signal is exploited for information decoding.

4) Power Splitting Based Receiver: In the power splitting (PS) based receiver architecture, after being received, the RF signal is split by a power splitter for supporting independent energy and information reception, as illustrated in Fig.23.

We still consider a generic MIMO channel for the case of the power splitting approach, where we have $N_{t}$ transmit antennas and $N_{r}$ receive antennas. The MIMO channel can be characterised by the $\left(N_{r} \times N_{t}\right)$-element matrix $\mathbf{H}$. Let us assume that the symbols transmitted by the transmitter are hosted by a $N_{t} \times 1$ column vector $\mathbf{x}$ and that the channel coefficients in $\mathbf{H}$ remain unchanged within a single symbol duration. Then we can derive the following expression for characterising the symbol received by the MIMO receiver:

$$
\mathbf{y}=\mathbf{H x}+\mathbf{I}+\mathbf{z}_{0}+\mathbf{z}_{c o v}
$$

where $\mathbf{I}$ is a $\left(N_{r} \times 1\right)$-element complex column vector representing the complex-valued interference at the receiver, $\mathbf{z}_{0}$ is a $\left(N_{r} \times 1\right)$-element complex column vector representing the complex AWGN imposed on the receive antennas with a power of $\sigma_{0}^{2}$ and $\mathbf{z}_{c o v}$ is also a $N_{r} \times 1$ complex column vector representing the complex AWGN having a power of $\sigma_{c o v}^{2}$ introduced by the RF-band to baseband converter. Similar to the spatial splitting based receiver architecture of SectionV-B-3), we have $\mathbf{H}=\mathbf{U} \boldsymbol{\Lambda} \mathbf{V}^{\dagger}$ by carrying out the SVD of $\mathbf{H}$, where the diagonal matrix $\boldsymbol{\Lambda}$ holds all the singular values of H. These singular values are denoted by $\left\{\lambda_{i} \mid 1 \leq i \leq \operatorname{rank}(\mathbf{H})\right\}$. Upon substituting $\mathbf{y}_{s v d}=\mathbf{U}^{\dagger} \mathbf{y}$ and $\mathbf{x}_{s v d}=\mathbf{V}^{\dagger} \mathbf{x}$ as well as $\mathbf{I}_{s v d}=\mathbf{U}^{\dagger} \mathbf{I}, \mathbf{z}_{0, s v d}=\mathbf{U}^{*} \mathbf{z}_{0}$ and $\mathbf{z}_{c o v, s v d}=\mathbf{U}^{\dagger} \mathbf{z}_{c o v}$ into Eq.(18), we have

$$
\mathbf{y}_{s v d}=\Lambda \mathbf{x}_{s v d}+\mathbf{z}_{0, s v d}+\mathbf{z}_{c o v, s v d},
$$

where $\mathbf{U}$ and $\mathbf{V}$ are both unitary matrices and hence multiplying the symbol vectors $\mathbf{y}, \mathbf{x}$ and $\mathbf{z}_{0}$ as well as $\mathbf{z}_{c o v}$ by them will not alter their statistical properties. Furthermore, the SVD virtually decompose the MIMO channel into several independent eigen-channels. As a result, the capacity of the MIMO channel $\mathbf{H}$ can be expressed as

$$
\begin{aligned}
& R^{P S}\left(\mathbf{P}_{\text {svd }}, \boldsymbol{\rho}_{\text {svd }}\right) \\
& =\sum_{i=1}^{\operatorname{rank}(\mathbf{H})} \log _{2}\left(1+\frac{\left(1-\rho_{s v d, i}\right) \lambda_{i}^{2} P_{s v d, i}}{\left(1-\rho_{s v d, i}\right)\left(\left|I_{s v d, i}\right|^{2}+\sigma_{0}^{2}\right)+\sigma_{c o v}^{2}}\right),
\end{aligned}
$$

where $\mathbf{P}_{s v d}=\left(P_{s v d, 1}, \cdots, P_{s v d, \operatorname{rank}(\mathbf{H})}\right)^{T}$ and $\boldsymbol{\rho}_{s v d}=$ $\left(\rho_{s v d, 1}, \cdots, \rho_{s v d, \operatorname{rank}(\mathbf{H})}\right)^{T}$ contain the transmit powers and the power splitting factors for the corresponding eigen-channels, respectively. Specifically, in the $i$-th eigen-channel, the portion $\rho_{s v d, i}$ of the received signal is exploited for energy harvesting, while the portion $\left(1-\rho_{s v d, i}\right)$ of the received signal is relied upon 
for information decoding. The eigenvalue $\lambda_{i}$ can be regarded as the coefficient of the $i$-th eigen-channel. Furthermore, the total energy received from the RF signal can be derived as

$$
P_{E}^{P S}\left(\mathbf{P}_{s v d}, \boldsymbol{\rho}_{s v d}\right)=\eta_{e} \sum_{i=1}^{\operatorname{rank}(\mathbf{H})} \rho_{s v d, i}\left(\lambda_{i}^{2} P_{s v d, i}+\left|I_{s v d, i}\right|^{2}+\sigma_{0}^{2}\right),
$$

where the constant $\eta_{e}$ characterises the RF-DC conversion efficiency. The signal splitting factor $\rho$ of the power splitting based receiver can be further calculated as

$$
\rho=\frac{\rho_{s v d, 1} P_{s v d, 1}+\rho_{s v d, 2} P_{s v d, 2}+\cdots+\rho_{s v d, i} P_{s v d, i}}{P_{s v d, 1}+P_{s v d, 2}+\cdots+P_{s v d, i}} .
$$

In the above-mentioned model, power splitting operates after the received signal of the multiple antennas is multiplied by a unitary matrix $\mathbf{U}^{\dagger}$. This is equivalent to splitting the received signals of each eigen-channel in the power domain.

There is another pair of power splitting based receiver architectures, namely independent power splitting [181] and uniform power splitting [182]. For the independent power splitting, the received signal of each antenna is directly split into two portions for energy harvesting and information decoding, respectively, without any signal processing operation. Furthermore, the antenna selection based spatial splitting receiver architecture of Section V-B-3) can also be regarded as a special independent power splitting, when the signal splitting factor at each antenna is either 0 or 1 . For the uniform power splitting, the received signals of multiple antennas are firstly combined and the aggregate signal is then split in the power domain for dedicated energy harvesting and information detection as well as decoding.

The power splitting based receiver architecture has been widely adopted [163], [164], [181], [183], [184] for integrating WET and WIT during downlink transmissions. Liu et al. [163] have proposed a dynamic power splitting based receiver architecture and they have demonstrated that a dynamic power splitting based receiver outperforms its fixed splitting-based counterpart. Furthermore, Zhang et al. [164] have characterised the rate-energy region of the power splitting based receiver and compared it ot its spatial splitting based counterpart. Li et al. [181] have considered the interference-contaminated SIMO system for integrated WET and WIT. Their results have revealed that the independent power splitting outperforms its uniform counterpart. Moreover, in full-duplex relay networks, the power splitting based receiver architecture is invoked by relays in order to simultaneously receive both energy and information from the sources [183]. The energy received may be depleted by the relays during altruistic data forwarding to their destination. Physical layer security is discussed in the context of a multiple-input-single-output (MISO) system in [184], where power splitting based receiver architecture is exploited for simultaneously receiving both information and energy.

The most important module of the power splitting based receiver architecture is the power splitter. The design of efficient power splitters at the circuit level has attracted enormous interest from the electronics engineering community. For example, Lee et al. [185] proposed a 1-V wideband CMOS phase and power splitter (PPS) relying on an RLC network load

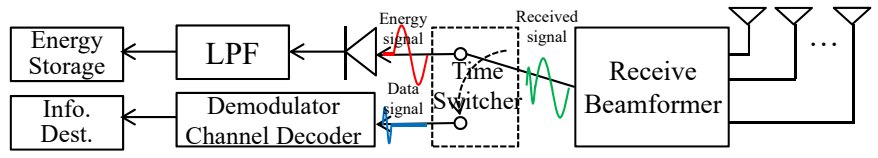

Fig. 24. Time switching based receiver architecture. The received signal is split in the time domain. The receiver firstly opts to exploit the received signal for information decoding. Then the receiver switches to the energy harvesting mode and the remaining signal is exploited for charging the energy storage unit.

and frequency compensation capacitor. This power splitter exhibited 7 degree phase error and $1.4 \mathrm{~dB}$ power imbalance. Chang et al. of [186] presented a V-band active one-to-four power splitter implemented in a 90-nm LP CMOS process for a phased-array transmitter. In their power splitter, 0.75 $\mathrm{dB}$ power imbalance and 4.3 degree phase imbalance were achieved. A power splitter operating in the millimetre wave frequency band was developed in [187] by Franc et al.. In their design, the so-called slow-wave transmission lines lead to a volume reduction of $70 \%$ compared to a classical power splitter designed with the aid of microstrip [188] or coplanar waveguide [189].

5) Time Switching Based Receiver: In the time switching (TS) based receiver, after the MIMO receiver, the signal is split in the time domain by a time switcher for independent energy and information receptions, as illustrated in Fig.24.

In contrast to the complexity of the power splitter of Fig.23, we only have to divide a typical transmission frame into two parts, namely into the energy transfer frame and the information transfer frame. The RF signal received during the energy transfer frame is delivered to the energy reception components exemplified by Fig.24 in order to convert the energy of the RF signal to DC, while the RF signal received during the information transfer frame is delivered to the information reception components in order to recover the original information.

In the time switching scheme, we consider the same received MIMO signal model in Eqs.(18) and (19). As a benefit of its flexible implementation, we can approximately tune the power splitter for each eigen-channel in order to maximise the information transmission rate or the amount of energy received by the power splitting scheme, as expressed in the optimisation problem of Eq.(29). By contrast, having diverse time switching factors for each eigen-channel is not quite realistic, since this operation may result in the time domain misalignment of the RF signals components received by the independent eigenchannels. Hence, additional synchronisation techniques are required, if we want to assign a specific time switching factor for each eigen-channel, which may significantly complicate the structural design of the transmission frame and the actual implementation of the diverse tunable time switcher.

As a result, we consider an identical time switching factor for all the independent eigen-channels, which indicates that only a single time switcher is required, as shown in Fig.24. Considering a time switching factor of $\rho$, the amount of power that can be gleaned from a portion of the received RF signal 


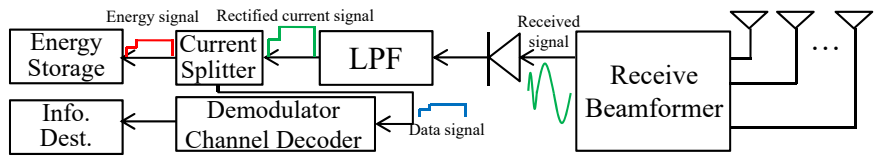

Fig. 25. Current Splitting based Receiver. After rectifying, the received signal lose its phase and frequency. The rectified current signal is split into two portions. One for charging the energy storage unit and the other for information decoding.

to the $\mathrm{DC}$ is expressed as

$$
P_{e}^{T S}\left(\mathbf{P}_{s v d}, \rho\right)=\eta_{e} \cdot \rho \cdot \sum_{i=1}^{\operatorname{rank}(\mathbf{H})}\left(\lambda_{i}^{2} P_{s v d, i}+\left|I_{s v d, i}\right|^{2}+\sigma_{0}^{2}\right) .
$$

Furthermore, the rest of the received RF signal is exploited for information delivery. As a result, the achievable information transmission rate can be derived as

$$
R^{T S}\left(\mathbf{P}_{s v d}, \rho\right)=(1-\rho) \sum_{i=1}^{\operatorname{rank}(\mathbf{H})} \log _{2}\left(1+\frac{\lambda_{i}^{2} P_{s v d, i}}{\left|I_{s v d, i}\right|^{2}+\sigma_{0}^{2}+\sigma_{c o v}^{2}}\right),
$$

where $\mathbf{P}_{s v d}=\left(P_{s v d, 1}, \cdots, P_{s v d, \operatorname{rank}(\mathbf{H})}\right)^{T}$.

Given its convenient implementation, the time switching based receiver architecture has attracted plenty of interests in the context of integrated WET and WIT [162], [190]-[193]. The rate-energy region of the time switching based receiver architecture has been characterised by Zhou et al. in [162] for a single-input-single-output (SISO) IDEN system. Zhu et al. [190] have investigated the optimal power control scheme of time switching based receivers in the context of a twouser interference channel. Stochastic optimisation theory has been exploited by Dong et al. in [191] for jointly addressing the power allocation and the splitting factor selection issues of time switching based receivers, when heterogeneous traffic patterns are encountered. The time switching based receiver architecture has also been widely adopted in wireless relay networks [192], [193]. For example, a relay selection scheme was jointly designed with the selection of time switching factors by Atapattu et al. in [192] for the sake of optimising outage probabilities and end-to-end data throughput. Furthermore, sub-carriers allocation as well as selection of time switching factors have been jointly optimised for multiple transmitterreceiver pairs [193].

6) Current Splitting Based Receiver: In the current splitting (CS) based receiver, as depicted in Fig.25, the RF signals are converted by a Schottky diode and a LPF to the DC signals after being received by the antennas. The major technical challenge in the current splitting based receiver architecture is to recover the information conveyed by the amplitude or energy pattern modulated signals. The current splitting based receiver architecture was originally proposed by Zhou $e t$ al. in [162]. In the rest of this section, we will briefly introduce the methodology of [162] for exploring the relationship between the DC signal $i_{D C}(t)$ and the RF signal $y(t)$. For simplicity, we consider a SISO transceiver for augmenting the rationale behind the information recovery from the DC signal. The RF signal $y(t)$ received by the antenna is expressed as

$$
y(t)=\sqrt{2} \mathcal{R}\left\{\sqrt{h P_{t}} x(t) e^{j(2 \pi f t+\theta)}+z_{0}(t) e^{j 2 \pi f t}\right\},
$$

where $x(t)=A(t) e^{j \phi(t)}$ is a complex symbol, whose amplitude $A(t)$ and phase $\phi(t)$ are modulated, $f$ is the carrier frequency of the RF signal, $P_{t}$ is the average transmit power, while $h$ and $\theta$ are the power gain and phase shift of the fading channel, respectively, where as $z_{0}(t)$ represents the antenna noise.

Substituting $y(t)$ of Eq.(25) into Eq.(13) and filtering out the high-frequency upper harmonic components by the LPF, the output DC current is expressed as

$$
\begin{aligned}
i_{D C}(t) \approx k_{0} & +k_{2}\left|\sqrt{h P_{t}} A(t)+z_{0}(t)\right|^{2} \\
& +k_{4}\left|\sqrt{h P_{t}} A(t)+z_{0}(t)\right|^{4}+z_{\text {rec }}(t),
\end{aligned}
$$

where the coefficients $\left\{k_{0}, k_{2}, k_{4}\right\}$ are the constant parameters of the Schottky diode used as the rectifier, while $z_{\text {rec }}(t)$ is the processing noise imposed by the Schottky diode and the LPF.

As shown in Fig.25, $i_{D C}(t)$ is split into two portions by a current splitter. The DC signal $\sqrt{\rho} i_{D C}(t)$ flows into batteries for energy storage, while the DC signal $i_{D C}(t) \sqrt{1-\rho}$ is passed onto the information decoder for recovering the original information bits. However, as demonstrated by Eq.(26), in the DC signal $i_{D C}(t)$, only the amplitude $A(t)$ of the original symbol $x(t)$ is retained. As a result, if the transmitter were to modulate the information by the classic quadrature-amplitudemodulation (QAM) or phase-shift-keying (PSK) scheme, the information carried by the phase $\phi(t)$ would be lost. Consequently, for the current splitting based receiver architecture, the transmitter has to modulate the information onto the energy pattern. Owing to this, we lost a precious degree of freedom for information transmission, hence the current splitting based receiver may not be able to support high-rate communication service, but as a benefit, we need lower signal processing power than a QAM-receiver.

For the energy reception, the DC signal $\sqrt{\rho} i_{D C}(t)$ can be exploited for charging the energy storage unit. If the energy $P_{e}^{C S}(t)$ stored in the battery is also proportional to $\sqrt{\rho} i_{D C}(t)$ with a coefficient of $\eta_{b a t}$, we have $P_{e}^{C S}=\eta_{b a t} \sqrt{\rho \mathbb{E}}\left[i_{D C}(t)\right]$, where $\mathbb{E}\left[i_{D C}(t)\right]$ represents the average of the DC rectified.

As a landmark contribution, Zhou et al. [162] have compared a power splitting based receiver and a current splitting based receiver in the context of a SISO system and they have demonstrated that the power splitting based receiver is preferable when the load circuit's power consumption is low, while the current splitting based receiver performs better, when the load circuit's power consumption is high.

7) Rate-Energy Tradeoff: For the signal splitter base receivers of Figs.22-25, a tradeoff has to be struck between the information rate and the amount of energy received. As the signal splitting factor $\rho$ increases, more RF-signal energy will be dedicated to energy reception, which inevitably diminishes the information rate. By contrast, as the signal splitting factor $\rho$ reduces, more RF-signal energy will be used for information reception, which degrades the energy reception performance.

The achievable rate-energy (R-E) region is an important performance metric of the integrated WET and WIT. We can now obtain the rate-energy region of the power splitting based 


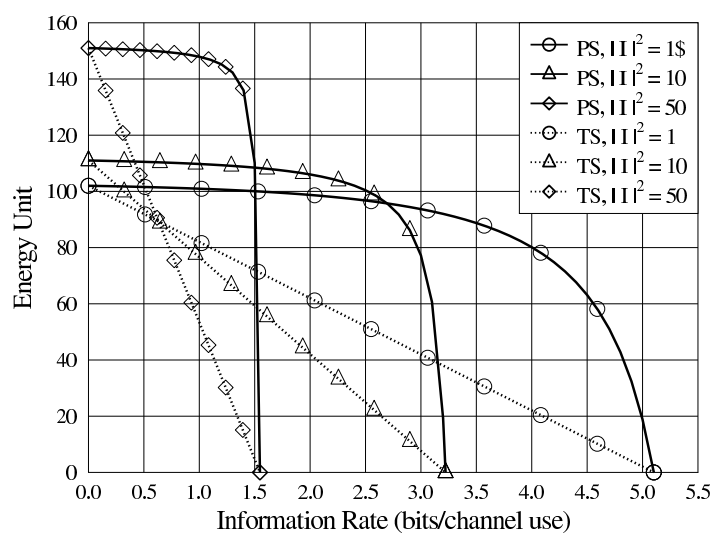

Fig. 26. Rate-energy tradeoff for the time switching based receiver and the power splitting based receiver in the SISO channel having $P_{t}=100,|h|^{2}=1$, $\sigma_{0}^{2}=1, \sigma_{\text {cov }}^{2}=1, \eta_{E}=1$ and $|I|^{2}=\{1,10,50\}$.

receiver with the aid of Eqs.(20) and (21) for the single-inputsingle-output (SISO) channel considered as

$$
\begin{array}{r}
C_{R-E}^{P S}\left(P_{t}\right) \triangleq \bigcup_{\rho}\left\{\left(R^{P S}, P_{E}^{P S}\right): P_{E}^{P S} \leq \eta_{e} \rho\left(|h|^{2} P_{t}+|I|^{2}+\sigma_{0}^{2}\right),\right. \\
\left.R^{P S} \leq \log _{2}\left(1+\frac{(1-\rho)|h|^{2} P_{t}}{(1-\rho)\left(|I|^{2}+\sigma_{0}^{2}\right)+\sigma_{c o v}^{2}}\right)\right\} .
\end{array}
$$

Similarly, with the aid of Eqs.(24) and (23), the rate-energy region of the time switching based receiver is derived for the SISO channel as

$$
\begin{array}{r}
C_{R-E}^{T S}\left(P_{t}\right) \triangleq \bigcup_{\rho}\left\{\left(R^{T S}, P_{E}^{T S}\right): P_{E}^{T S} \leq \eta_{e} \rho\left(|h|^{2} P_{t}+|I|^{2}+\sigma_{0}^{2}\right),\right. \\
\left.R^{T S} \leq(1-\rho) \log _{2}\left(1+\frac{|h|^{2} P_{t}}{|I|^{2}+\sigma_{0}^{2}+\sigma_{c o v}^{2}}\right)\right\} .
\end{array}
$$

Fig.26 clearly illustrates the rate-energy tradeoff for both the power splitting based receiver of Fig.23 and its time switching based counterpart of Fig.24. The parameters in Fig.26 are normalised by the AWGN power $\sigma_{0}^{2}$ at the receive antenna. We can observe from Fig.26 that the interference does not always impose adverse effects on the integrated WET and WIT system. For example, for the power splitting based receiver of Fig.23, if the desired information rate is as low as $1 \mathrm{bit} / \mathrm{channel}$ use, we can gain more energy when we have more interference at the receiver. Similarly for the time switching based receiver, the same trend can be observed if the desired information rate is as low as $0.5 \mathrm{bit} / \mathrm{channel}$ user. This observation offers us some hints on the implementation issues of the integrated WET and WIT. We can enhance the transmit power for the low-rate services, such as paging or for the control signalling exchange in cellular communications, in order to transfer more energy to the receiver. In this scenario, the energy reception benefits from the inter-cell interference or other types of interference without violating the quality of the low-rate service.

In this section, we investigate the rate-energy tradeoff without considering the sensitivities of the practical energy harvester and the information receiver. However, studying the rate-energy tradeoff is still essential for the integrated WIT and WET by considering practical energy harvesters and information receivers, although their sensitivities have several orders of magnitude difference, as we have stated in Section I-E. The reasons are summarised as follows:

- The energy harvesting performance is determined by the absolute value of the received RF signal power, which is aggregated by the useful signal power $|h|^{2} P_{t}$, the interference power $|I|^{2}$ and the antenna noise power $\sigma_{0}^{2}$, as expressed in Eqs.(27) and (28). The energy carried by the received RF signal can only be harvested, if its power is higher than the sensitivity threshold of the energy harvester.

- The information receiver's performance is dominated by the received SINR. For example, in a multi-tier heterogeneous cellular network, the interference power is most likely to be $-20 \mathrm{dBm}$ [63]. The interference power alone is sufficient for activating the information receiver having a sensitivity of $-72 \mathrm{dBm}$ [61]. However, we have to allocate sufficient received RF signal power for recovering the information bits contaminated by the interference and the noise $n_{c o v}$ generated by the passband to baseband converter.

\section{MIMO aided IDEN System}

Multiple-input-multiple-output (MIMO) techniques have been widely adopted for both WET and WIT [164], [165], since multiple antennas are capable of focusing energy for creating a directional beam and they are also capable of providing spatial multiplexing gains. Specifically, in an IDEN, a single antenna may not be capable of satisfying the energy and data demands of UEs. Hence, MIMO systems have attracted much interest in enabling integrated WET and WIT [194]-[197].

1) Joint Transceiver Design: A transmitter equipped with a single isotropic antenna fails to transfer sufficient energy to the far-field due to the adverse effects of path-loss. However, if multiple antennas are invoked at the transmitters, a focused energy beam can be formed by acquiring the knowledge of the channel state information (CSI) for the sake of counteracting the wireless channel's attenuation. Beamforming allows us to control the direction of RF signals transmitted by each antenna by adjusting both the signals' amplitudes and phases. As a result, the energy can be focused in a directional beam for providing charging and data delivery services to UEs in the target region. Furthermore, efficient signal splitting strategies have to be adopted by the receivers in order to maximise both the amount of received energy and the amount of received data. Only the joint design of the WIT and WET transceiver is capable of maximising performance of integrated WET and WIT. Upon considering the power splitting based receiver architecture of Fig.23, as introduced in Section V-B4 , the following optimisation problem may be formulated for characterising the joint beamformer and signal splitting design 
TABLE VIII

Summary of Physical Techniques for IDENs

\begin{tabular}{|c|c|c|c|c|c|}
\hline Ref. & Receiver architecture & System model & Channel model & Optimal object & $\begin{array}{r}\text { CSI } \\
\text { requirement }\end{array}$ \\
\hline $\begin{array}{l}\text { Zhou } \\
\text { et al. [162] }\end{array}$ & $\begin{array}{l}\text { Time Switching } \\
\text { Power Splitting } \\
\text { Current Splitting }\end{array}$ & $\begin{array}{r}\text { Point-to-point } \\
\text { SISO }\end{array}$ & AWGN & $\begin{array}{r}\text { Rate-energy } \\
\text { region }\end{array}$ & N.A. \\
\hline $\begin{array}{l}\text { Zhang } \\
\text { et al. }[165]\end{array}$ & $\begin{array}{r}\text { Time Switching } \\
\text { Spatial Splitting } \\
\text { Uniform power splitting } \\
\text { Independent power splitting }\end{array}$ & $\begin{array}{r}\text { Point-to-point } \\
\text { MIMO }\end{array}$ & Rayleigh & $\begin{array}{r}\text { Rate-energy } \\
\text { region }\end{array}$ & Perfect \\
\hline $\begin{array}{l}\text { Zhu } \\
\text { et al. [190] }\end{array}$ & Fixed time Switching & $\begin{array}{r}\text { Two-user } \\
\text { MIMO }\end{array}$ & $\begin{array}{r}\text { Rayleigh } \\
\text { Interference channel }\end{array}$ & Sum-throughput & Perfect \\
\hline $\begin{array}{l}\text { Dong } \\
\text { et al. }[191]\end{array}$ & Time Switching & $\begin{array}{r}\text { K-user } \\
\text { SISO }\end{array}$ & $\begin{array}{r}\text { Rayleigh } \\
\text { Orthogonal channels }\end{array}$ & $\begin{array}{r}\text { Average transmit } \\
\text { power }\end{array}$ & N.A. \\
\hline $\begin{array}{l}\text { Atapattu } \\
\text { et al. }[192]\end{array}$ & Time Switching & $\begin{array}{r}\text { Single-relay } \\
\text { SISO and } \\
\text { Multi-relay } \\
\text { SISO }\end{array}$ & Fading channels & $\begin{array}{r}\text { SNR } \\
\text { Outage probability }\end{array}$ & Perfect \\
\hline $\begin{array}{l}\text { Shen } \\
\text { et al. [193] }\end{array}$ & Time Switching & $\begin{array}{r}\text { OFDM relay } \\
\text { SISO }\end{array}$ & AWGN & Sum-throughput & N.A. \\
\hline $\begin{array}{l}\mathrm{Li} \\
\text { et al. }[181]\end{array}$ & Independent Power Splitting & SIMO & $\begin{array}{r}\text { Rayleigh } \\
\text { Interference channels }\end{array}$ & $\begin{array}{r}\text { Rate-energy } \\
\text { region }\end{array}$ & $\begin{array}{r}\text { Perfect CSI } \\
\text { statistical CSI }\end{array}$ \\
\hline $\begin{array}{l}\text { Liu } \\
\text { et al. [183] }\end{array}$ & Power Splitting & $\begin{array}{l}\text { Full-duplex } \\
\text { relay SISO }\end{array}$ & $\begin{array}{r}\text { Rayleigh } \\
\text { Self-interference } \\
\text { channels }\end{array}$ & $\begin{array}{r}\text { End-to-end } \\
\text { SINR }\end{array}$ & Perfect \\
\hline $\begin{array}{l}\text { Chu } \\
\text { et al. [184] }\end{array}$ & Power Splitting & $\begin{array}{r}\text { Point-to-point } \\
\text { MISO } \\
\end{array}$ & Rayleigh & Transmit power & Imperfect \\
\hline $\begin{array}{l}\text { Timotheou } \\
\text { et al. [194] }\end{array}$ & Power Splitting & $\begin{array}{r}\text { Point-to-point } \\
\text { MISO }\end{array}$ & Rayleigh & Transmit power & Perfect \\
\hline $\begin{array}{l}\text { Liu } \\
\text { et al. [195] }\end{array}$ & $\begin{array}{r}\text { Conventional WET receiver } \\
\text { and WIT receiver }\end{array}$ & $\begin{array}{r}\text { Secrecy } \\
\text { broadcasting } \\
\text { MISO }\end{array}$ & $\begin{array}{r}\text { Rayleigh } \\
\text { Interference channel }\end{array}$ & Secrecy rate & Perfect \\
\hline $\begin{array}{l}\text { Shi } \\
\text { et al. [196] }\end{array}$ & Power Splitting & $\begin{array}{r}\text { Multi-user } \\
\text { MISO }\end{array}$ & $\begin{array}{c}\text { Rayleigh } \\
\text { Interference channel }\end{array}$ & Transmit power & PerfectI \\
\hline $\begin{array}{l}\text { Wen } \\
\text { et al. [197] }\end{array}$ & Power Splitting & $\begin{array}{l}\text { Full-duplex } \\
\text { relay MISO }\end{array}$ & $\begin{array}{r}\text { Rayleigh } \\
\text { Self-interference } \\
\text { channel }\end{array}$ & $\begin{array}{r}\text { Mean square } \\
\text { error }\end{array}$ & Perfect \\
\hline $\begin{array}{l}\text { Cirik } \\
\text { et al. [198] }\end{array}$ & Uniform Power Splitting & $\begin{array}{r}\text { Full-duplex } \\
\text { multi-user } \\
\text { MIMO }\end{array}$ & $\begin{array}{r}\text { Rayleigh } \\
\text { Interference channel } \\
\text { Self-interference } \\
\text { channel }\end{array}$ & Transmit power & Perfect \\
\hline $\begin{array}{l}\text { Zhao } \\
\text { et al. [199] }\end{array}$ & Independent Power Splitting & $\begin{array}{r}\text { Full-duplex } \\
\text { multi-user } \\
\text { MIMO }\end{array}$ & $\begin{array}{r}\text { Rayleigh } \\
\text { Interference channel } \\
\text { Self-interference } \\
\text { channel }\end{array}$ & $\begin{array}{l}\text { Transmit power } \\
\text { Sum-throughput }\end{array}$ & Perfect \\
\hline $\begin{array}{l}\text { Park } \\
\text { et al. [200] }\end{array}$ & $\begin{array}{r}\text { Conventional WET receiver } \\
\text { and WIT receiver }\end{array}$ & $\begin{array}{l}\text { Two-user } \\
\text { MIMO }\end{array}$ & $\begin{array}{r}\text { Rayleigh } \\
\text { Interference channel }\end{array}$ & $\begin{array}{r}\text { Sum-throughput } \\
\text { Energy harvested }\end{array}$ & Perfect \\
\hline $\begin{array}{l}\text { Shen } \\
\text { et al. [201] }\end{array}$ & $\begin{array}{r}\text { Conventional WET receiver } \\
\text { and WIT receiver }\end{array}$ & $\begin{array}{r}\text { Multi-user } \\
\text { MISO }\end{array}$ & $\begin{array}{r}\text { Rayleigh } \\
\text { Interference channel }\end{array}$ & Sum-throughput & Perfect \\
\hline $\begin{array}{l}\text { Koo } \\
\text { et al. [202] }\end{array}$ & Uniform Power Splitting & $\begin{array}{r}\text { Multi-user } \\
\text { MIMO }\end{array}$ & $\begin{array}{r}\text { Rayleigh } \\
\text { Interference channel }\end{array}$ & $\begin{array}{r}\text { Rate-energy } \\
\text { region }\end{array}$ & Perfect \\
\hline $\begin{array}{l}\text { Zong } \\
\text { et al. [203] }\end{array}$ & Uniform Power Splitting & $\begin{array}{r}\text { Multi-user } \\
\text { MIMO }\end{array}$ & $\begin{array}{r}\text { Rayleigh } \\
\text { Interference channel }\end{array}$ & Transmit power & $\begin{array}{r}\text { Exchanging } \\
\text { CSI }\end{array}$ \\
\hline
\end{tabular}

of a MIMO aided IDEN system, as

$$
\begin{aligned}
\text { Objective: } & \max _{\mathbf{P}_{s v d}, \boldsymbol{\rho}} R^{P S}\left(\mathbf{P}_{s v d}, \boldsymbol{\rho}\right), \\
\text { Subject to: } & P_{E}^{P S}\left(\mathbf{P}_{s v d}, \boldsymbol{\rho}\right) \geq P_{E, t h}, \\
& \sum_{i=1}^{\operatorname{rank}(\mathbf{H})} P_{s v d, i} \leq P_{t, \text { total }},
\end{aligned}
$$

where the expressions of $R^{P S}\left(\mathbf{P}_{s v d}, \boldsymbol{\rho}\right)$ and $P_{E}^{P S}\left(\mathbf{P}_{s v d}, \boldsymbol{\rho}\right)$ are given by Eqs.(20) and (21), respectively. This optimisation problem aims for maximising the achievable data throughput, as interpreted in terms of its objective function (29). The constraint (29a) represents that the received energy should be higher than the threshold $P_{E, t h}$, while (29b) represents that the sum of the allocated power should not exceed the total available transmit power $P_{t, \text { total }}$. In order to solve the associated practical implementation issues, we have to convert the optimal virtual transmit power $\mathbf{P}_{s v d}^{*}$ and the power splitting factor $\boldsymbol{\rho}_{s v d}^{*}$ of the eigen-channels into the actual transmit power $\mathbf{P}_{t}^{*}$ of the transmit antennas and the power splitting factor $\boldsymbol{\rho}^{*}$ of the receive antennas with the aid of the equations $\mathbf{P}_{t}^{*}=|\mathbf{V}|^{2} \mathbf{P}_{s v d}^{*}$ and $\boldsymbol{\rho}^{*}=|\mathbf{U}|^{2} \boldsymbol{\rho}_{s v d}^{*}$. The optimal power $\mathbf{P}_{t}^{*}$ of the transmit antennas can be regarded as that of the optimal transmit beamformer, while the optimal power splitting factors $\rho^{*}$ can be regarded as the optimal strategy adopted by the receiver. As a result, this optimisation problem defines a joint transceiver design. Similar optimisation problems can also 
be formulated for spatial splitting and time switching based receiver architectures.

Using beamforming is essential for a MIMO-aided IDEN system. Xiang et al. [165] have considered a scenario consisting of a BS equipped with multiple antennas, a WIT-UE and a WET-UE. The beamformer of the BS is optimised in order to maximise the energy received by the WET-UE, while satisfying the minimum data throughput requirement of the WIT-UE. Both the perfect and imperfect CSI knowledge have been taken into account. Furthermore, beamforming was also conceived for integrated WET and WIT in [164] for a MIMO aided point-to-point channel. Moreover, beamforming schemes were also designed for spatial splitting, uniform power splitting and independent power splitting based receivers for maximising the energy harvested subject to data throughput constraints by relying on the ideal assumption of perfect CSI. In a multi-user MISO inteference channel, Timotheou et al. [194] have studied both the cases of separate fixed beamforming and when the beamforming design is jointly optimised with the power splitting based receivers. Their numerical results have demonstrated that - as expected - the joint optimisation outperforms the separate one, although it requires a highcomplexity algorithm. By focusing on the confidentiality of WIT, secure IDEN has also been studied by Liu et al. [195], where the optimal beamformer has been designed for maximising the WIT-UEs secrecy rate, while ensuring the minimum energy requirement of the WET-UEs. Furthermore, beamformer designs have also been investigated by Liu et al. [196] in IDENs for supporting multiple WET and WIT UEs by considering a MISO interference channel. Wen et al. [197] have studied a full-duplex MIMO aided relay by adopting the classic amplify-and-forward protocol. Initially, the source supports integrated WET and WIT for the relay. The relay then forwards data to its destination by exploiting the energy received from the source. The beamformers of the source and relay are jointly designed in order to minimise the mean-square-error of the data received by the destination.

2) Multi-User MIMO: Apart from beamforming, MIMOs are also capable of supporting multiple UEs' energy charging and data delivery requests by exploiting spatial division multiplexing.

When multiple antennas are invoked at a transmitter, the antenna array may be split into $K$ antenna groups in order to support $K$ UEs at the same time within the same frequency band. In the MIMO aided spatial division multiplexing, signalto-interference-plus-noise-ratio (SINR) becomes a critical metric fundamentally determining the information rate, while the interference becomes an additional precious energy source for energy-thirsty UEs.

Considering a $K$-UE MIMO aided IDEN system, let $\mathbf{h}_{i, j}$ denote the channel's power gains between the $i$-th transmit antenna group and $j$-th UE, when the uniform power splitting based receiver architecture is adopted by the UEs. Therefore, the SINR of the $i$-th UE may be expressed as

$$
S I N R_{i}=\frac{\left(1-\rho_{i}\right) \operatorname{Tr}\left(\mathbf{h}_{i, i}^{\dagger} \mathbf{x}_{i} \mathbf{h}_{i, i}\right)}{\sum_{j \neq i}^{K}\left(1-\rho_{i}\right) \operatorname{Tr}\left(\mathbf{h}_{j, i}^{\dagger} \mathbf{x}_{j} \mathbf{h}_{j, i}\right)+\sigma_{0}^{2}+\sigma_{c o v}^{2}},
$$

where $\mathbf{x}_{i}$ represents the symbols transmitted from the $i$-th transmit antenna group, which are destined to the $i$-th UE, $\sigma_{0}^{2}$ is the noise power and $\sigma_{c o v}^{2}$ is the noise power of the passband to baseband converter, whereas $\rho_{i}$ is the uniform power splitting factor adopted by the $i$-th UE. Furthermore, the energy $P_{E, i}$ harvested by the $i$-th UE, which is measured in terms of Watt, may be formulated as

$$
P_{E, i}=\eta_{e} \rho_{i} \sum_{j=1}^{K} \operatorname{Tr}\left(\mathbf{h}_{j, i}^{\dagger} \mathbf{x}_{j} \mathbf{h}_{j, i}+\sigma_{0}^{2}\right)
$$

where a linear RF-DC converter having a conversion efficiency $\eta_{e}$ is employed.

One of the optimisation problems in a MIMO aided IDEN supporting $K$-UEs, can be formulated as:

$$
\begin{aligned}
\text { Objective: } & \min _{\mathbf{X}, \rho_{i}} \operatorname{Tr}\left(\mathbf{X} \mathbf{X}^{\dagger}\right), \\
\text { Subject to: } & S I N R_{i} \geq \gamma_{t h, i}, \\
& P_{E, i} \geq P_{t h, i}, \\
& 0 \leq \rho_{i} \leq 1,
\end{aligned}
$$

where $i=1, \cdots, K$ and the column vector $\mathbf{X}$ is a collection of the symbols transmitted by all antennas. For example, the symbols transmitted by the $i$ th group of antennas are denoted by a vector $\mathbf{x}_{i}$. If the $n$-th antenna is categorised into the $i$-th group, its transmitted symbol $x_{n}$ belongs to the vector $\mathbf{x}_{i}$. As a result, the $n$-th element of $\mathbf{X}$ is equal to $x_{n}$. This optimisation problem aims for minimising the total transmit power of the transmitter, while ensuring that the SINR of the $i$-th UE exceeds a predefined threshold $\gamma_{i}$ and that the energy received by the $i$-th UE is also above a predefined threshold $P_{t h, i}$.

Note that the beamformer design of the multi-UE MIMO aided IDEN system is different from the conventional WIT system, since the interference, which is capable of providing additional energy, is no longer regarded as an adverse effect. As a result, the tradeoff between WET and WIT in multiUE scenarios should be carefully reconsidered. Park et al. [200] have investigated the joint WIT and WET in a twouser MIMO interference channel, while Shen et al. [201] have extended the two-user scenario to a multi-user one, where a pair of receiver architectures have been studied, namely the power splitting based receivers of Fig.23, time switching based receivers of Fig.24 using time-division-multiple-access protocols. Furthermore, Koo et al. [202] have investigated integrated WET and WIT in a multi-user MIMO scenario, where the interference is aligned at the reduced signal subspace at each receiver. Moreover, Zong et al. [203] have investigated the transceiver design problems of power splitting enabled colocated WET and WIT in MIMO interference channels. An efficient algorithm has been proposed based on semi-definite relaxation aided alternating optimization of the joint transmit beamformer, power splitters and receiver filters. Additionally, full duplex solutions have also been invoked in multi-user MIMO IDEN systems by Cirik et al. [198] as well as Zhao et al. [199].

3) Massive MIMO: As a fundamental technique, which might bring about revolutionary changes in the physical layer 
design, massive MIMO systems are capable of operating fully coherently and adaptively by relying on a very large number of antennas (hundreds or thousands), which can also be exploited for efficient WET and WIT by forming extremely narrow beams. Apart from the obvious increase of WET and WIT efficiencies, massive MIMO systems also rely on lower-power transceiver components. They may achieve reduced latency and low-complexity media access control (MAC) layer [204]. Naturally, integrated WET and WIT is also able to benefit from these compelling massive MIMO techniques. Furthermore, with the aid of a large number of antennas, multiple UEs may simultaneously access the IDEN systems by exploiting the spatial-division-multiple-access (SDMA) protocol, where again, the interference incurred by multiple UEs may be ignored. As a result, both the data throughput and the energy harvested can be dramatically increased by adopting massive MIMO techniques.

Recently, researchers have invested their endeavour into massive MIMO aided WET and WIT system [204]-[207]. Yang et al. [205] have optimised the throughput of a WET enabled massive MIMO system by acquiring imperfect CSI knowledge. The minimum data rate among UEs has been maximised by jointly optimising the allocation of time and energy resources. Furthermore, the authors have also studied the impact of the number of antennas on system performance. They have found that having a relatively small number of antennas at the transmitter may achieve a favourable performance. Zhao et al. [206] have studied the optimal precoder design of a massive MIMO aided integrated WET and WIT system for the sake of maximising the minimum energy harvested by WET-UEs, while ensuring the data throughput of WIT-UEs. Furthermore, optimal beamformer design for massive MIMO aided IDEN system has been addressed by Zhao et al. [207].

\section{Waveform Design}

The exciting development of WET in electronic engineering has been largely overlooked by the community of communication engineering. Research in WET from the perspective of electronic engineering mainly focus on the WET efficiency, which is highly closed to the waveform design of RF signals radiated by transmitters [167]. By contrast, the communication society pay much more attention on the system-level architecture design and resource allocation for integrated WET and WIT. The circuit-level characteristics are simplified by assuming linear RF-DC converter. According to the natural behaviour of RF-DC converters, it has been demonstrated that multi-sine RF signals outperform single-carrier RF signals in terms of WET efficiency. This is because multi-sine RF signals having a higher PAPR than single-carrier ones are more suitable for activating RF-DC converters and for keeping RFDC converters operating in high efficiencies [208].

Orthogonal-frequency-division-multiplexing (OFDM) is a widely-adopted technique in both LTE aided cellular networks and WiFi aided WLANs, which is capable of generating multisine RF signals. We plot the CDF of PAPR for a 802.11a based OFDM system associated with the binary-phase-shiftkeying (BPSK) modulation in Fig.27. We observe from Fig.27

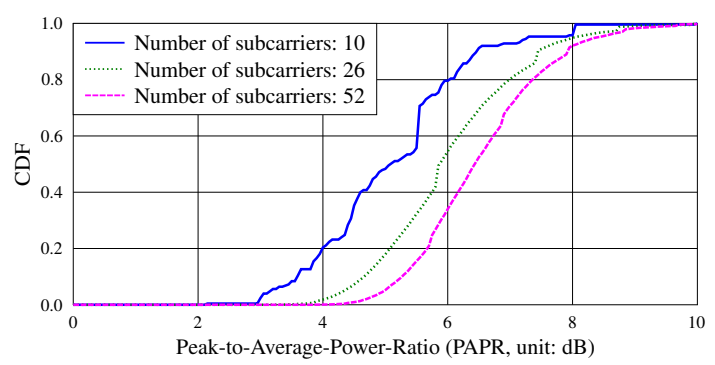

Fig. 27. The CDF of the PAPR of a 802.11a based OFDM system associated with the BPSK modulation. The size of the Fast-Fourier-Transformation (FFT) is 128 . The maximum number of subcarriers is 52 .

that if a higher number of subcarriers are allocated to a transmitter-receiver pair, the PAPR is substantially increased. Fig.27 demonstrated that OFDM is capable of providing a waveform of high PAPR, which may remarkably increase the energy delivery efficiency.

A large number of works [166]-[169], [209] have been done for characterising the relationship between waveform and WET efficiency. For example, a $K$-sine RF signal $x(t)$ can be expressed as $x(t)=\mathfrak{R}\left(\sum_{i=1}^{K} s_{i} e^{j\left(2 \pi f_{i} t+\phi_{i}\right)}\right)$, where $f_{i}=$ $f_{1}+\delta f$ and $\phi_{i}$ denote the frequency and the phase of the $i$-th sine waveform, respectively, and $s_{i}$ represents its amplitude. Multiplying $x(t)$ by a channel coefficient, we may obtain the received RF signal $y(t)$. According to Eqs.(13) and (14), the energy harvested by receivers is determined by both the frequencies and phases of the multi-sine signal $x(t)$. As a result, the waveform design can be formulated by the following optimisation problem:

$$
\begin{aligned}
& \text { Objective: } \max _{\mathbf{s}, \phi} \mathbb{E}\left[i_{D C}(t)\right] \\
& \text { Subject to: } \frac{1}{2} \sum_{i=1}^{K} s_{i}^{2} \leq P_{t},
\end{aligned}
$$

where the expression of $i_{D C}(t)$ is given by Eq.(13), $\mathbb{E}[\cdot]$ represents the expectation operation, the amplitudes and the phases of multiple sines signals are collected into the vectors $\mathbf{s}$ and $\boldsymbol{\phi}$, respectively. This problem aims for maximising the energy carried by the converted DC signal by carefully choosing the amplitudes $\mathbf{S}$ and the phases $\boldsymbol{\phi}$ for the multisine RF signals, which is subject to the total transmit power constraint.

However, WIT has totally different requirement on multisine RF signals from the perspective of information decoding. In a pure WIT system, engineers have to suppress the PAPR as low as possible in order to obtain a reasonable information decoding performance. Clearly, WIT and WET has a fundamental contradiction in the design principle. As a result, waveform has to be carefully designed by jointly considering contradicted requirements of WET and WIT in order to achieve an acceptable balance for IDEN systems. Kim et al. [169] have smartly dealt with this contradiction by encoding information bits on PAPRs, since a certain PAPR can be achieved by accumulating a certain amount of sine signals. For example, two sine signals may be accumulated and achieve 


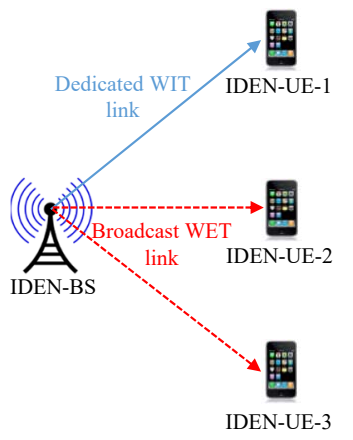

(a) A single BS scenario

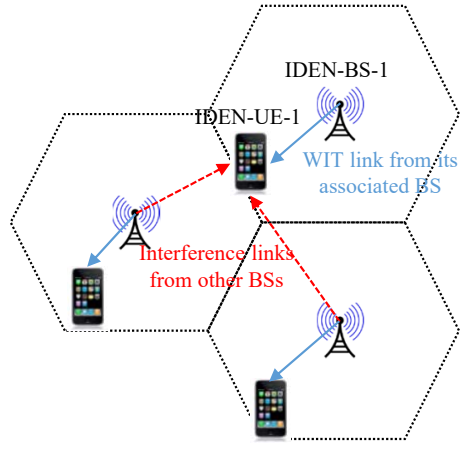

(b) A multiple BSs scenario
Fig. 28. Non-cooperative IDENs

a PAPR of two, while three sine signals may achieve a PAPR of 3. However, this modulation scheme may suffer from a big loss of information rate, since it cannot carry information bits by phases and frequencies of RF signals. Further effort should be invested in the waveform design for integrated WET and WIT.

\section{Resource Allocation and Protocol Design for MAC}

Relying on the judicious allocation of communication resources (e.g., frequency band, transmitter's power and time slot), we can improve the performance (e.g., the total throughput of the networks, user's throughput fairness and energy consumption of all transmitters) of the network and achieve the best possible exploitation of resources. In contrast to the resource allocation in conventional WIT networks, the tradeoff between data throughput and the harvested energy has to be considered in IDENs. Hence, resource allocation in IDENs constitutes a challenging research topic. We categorize the existing contributions into two classes: resource allocation in non-cooperative and cooperative IDENs.

On the other hand, a well-designed MAC protocol is capable of controlling the channel access of multiple UEs by efficiently exploiting both the energy harvested from RF signals and the precious communication medium. Hence, we will also discuss the design principles of MAC protocols for IDENs.

\section{A. Resource Allocation in Non-cooperative IDENs}

In non-cooperative IDENs, either there is only a single BS or there may in fact be multiple independent BSs, which do not share any information with each other, as portrayed in Fig.28.

In a single-BS scenario, orthogonal channels (e.g. subcarriers, time-slots and antennas) have to be allocated to multiple UEs for their dedicated downlink WIT [210]. Due to the broadcast nature of wireless channels, RF signals carried by dedicated WIT links are also capable of reaching other UEs, as illustrated in Fig.28(a). Since these signals are not delivered by the orthogonal channels allocated to them, these UEs do not carry out any information decoding operation. However, these RF signals can be exploited for energy harvesting in order to satisfy their charging requirements.
In a multiple-BS scenario, every UE is associated with its nearest BS for any communication service, as portrayed in Fig.28, while other BSs may be regarded as interferers. The interference imposed by other BSs on a UE may degrade its communication performance. However, the interference can also be exploited for supplying additional energy to the UEs. Since the interferers do not possess any knowledge about the UEs associated with other BSs, they cannot actively adapt their transmitters to satisfy the energy demand of the UEs. The UEs simply harvest energy passively from the ambient RF signals.

In this section, we will firstly discuss various resource allocation schemes in non-cooperative IDENs, which aims for throughput maximisation, fairness maximisation and energy consumption minimization. These resource allocation schemes are subject to the practical constraints on the amount of energy harvested and those on the attainable throughput.

1) Throughput Maximization: We firstly review the latest contributions concerning throughput maximisation, which aim for maximising the total throughput of multiple UEs in IDENs. Throughput maximisation problems are usually subject to some constraints, such as the minimum amount of energy harvested by UEs and the maximum transmit power of BSs.

Zhou et al. [211] as well as Yin et al. [212] have investigated the maximisation of the total throughput of multiple UEs subject to a minimum requirement on the energy harvested and subject to a peak and/or total transmit power constraint in the downlink of a multi-UE OFDM system. Specifically, the weighted sum-rate of all UEs has been maximised in [211] for guaranteeing the fairness among all the UEs, while maximising the conventional sum-rate has been the objective in [212]. In contrast to conventional WIT networks, where UEs only receive data information from BSs, it is assumed by Zhou et al. [211] and Yin et al. [212] that the UEs may simultaneously extract both data and energy from their received RF signals. Zhou et al. [211] have considered both TDMA and OFDMA for the access of multiple UEs. In the scenario supported by the classic TDMA protocol, time switching based receiver architectures are adopted by the UEs for simultaneously receiving the requested data and for charging their batteries. Relying on Lagrange duality [211], the optimal power allocation and the optimal splitting factors of time switching based receivers are determined. In the scenario supported by the OFDMA protocol, power splitting based receiver architectures are adopted by the UEs. The authors have proposed an iterative algorithm for jointly optimising both the power and the sub-carrier allocation, where the optimal splitting factors of power splitting based receivers are obtained when the algorithm converges. By extending the solutions of [211], Yin et al. [212] have proposed a sub-carrier separation scheme, where the information and energy are transferred separately on different sub-carriers. The sum-rate optimisation has been formulated as a mixed integer programming problem. Both optimal and suboptimal solutions have been obtained and they are compared to the time switching based scheme of [211]. Observe from the numerical results that the subcarrier separation scheme outperforms the time switching based scheme, especially when a high energy is required by the UEs. 
Wang et al. [213] have studied the sum-throughput maximisation problem by optimally allocating the time duration for the downlink WET and for the uplink WIT, where the "harvest-then-transmit protocol" is advocated for the batteryless UEs. In this protocol, the UEs first harvest energy emitted by a BS during its downlink transmission and then upload their data information to the BS in a TDMA manner during their uplink transmission by exploiting the energy harvested from the BS. Wang et al. [213] have firstly solved the sumthroughput maximisation problem. However, the doubly nearfar phenomenon exists in the "harvest-then-transmit protocol" aided system. In this system, a UE far from the BS receives less energy than a UE close to the BS, but this UE has to transmit at a higher power during its uplink transmission in order to achieve the same information rate as its peer close to the BS. Consequently, in order to achieve the maximum sumthroughput, more resources have to be allocated to the UEs closer to the BS than to those far away from the BS. Obviously, this allocation scheme ignores the fairness among UEs, since the UEs far away from the BS gain limited resources for accomplishing their uplink transmissions.

The resource allocation problems of multi-UE MIMO systems of integrate WET and WIT have been investigated by Wang et al. [214] as well as Rubio et al. [215]. Wang et al. [214] has studied a network consisting of $(K+L+1)$ nodes, in which a single BS is capable of transferring both data and energy to the UEs, where $K$ WIT UEs only decode the information carried by the RF signals, while $L$ WET UEs only harvest energy from the RF signals. Semi-closedform expressions have been obtained for characterising the rate-energy region of the system. Furthermore, the classic bisection search algorithm [214] has been exploited for finding the dual variables in order to optimise the power allocation problem. In [215], the transmit covariance matrix has been designed for MIMO broadcast networks of integrated WET and WIT. The problem has been formulated as a multiobjective optimisation problem, in which the information rates and the energy harvested have been optimised simultaneously. In order to solve this multi-objective optimisation problem, two scenarios have been considered. In the first scenario, the specific amount of the energy harvested by each UE can be controlled by the transmitter, whereas only a portion of the energy harvested by UEs can be controlled in the second scenario. Rubio et al. [215] have solved the associated multiobjective optimisation problems for these two scenarios by convex approximations.

Furthermore, in order to realise secure communications in the physical layer, Shi et al. [216] have maximised the secrecy rate subject to a specific total power constraint and an energy harvesting constraint by designing an optimal beamformer for a MIMO-aided broadcast system.

2) Fairness: Apart from the sum-throughput, fairness is another crucial metric to be considered in resource allocation for the integrated WET and WIT. Diverse criteria can be used for evaluating the fairness. For example, in [213] and [217], the minimum uplink throughput among all the UEs has been maximised for achieving the fairness. By contrast, in [218], the weighted sum-throughput has been maximised for achieving the fairness, where the weights of UEs represent their specific needs in resource allocation.

The doubly near-far phenomenon, which results in unfair uplink rates among UEs, has been investigated Wang et al. [213], when the "harvest-then-transmit" protocol is adopted for the downlink WET and the uplink WIT. In order to overcome the adverse effects of this phenomenon, Wang et al. [213] have maximised the so-called common-throughput so as to reduce the differences among the UEs' uplink transmission rates. Their numerical results have shown that the commonthroughput maximisation is capable of overcoming the doublynear-far phenomenon, albeit at a cost of some sum-throughput degradation.

The doubly-near-far phenomenon has also been considered by Liu et al. [217] and Ju et al. [218]. Similar to [213], Liu et al. [217] have maximised the minimum uplink throughput among all UEs. However, the UEs upload their data to the BS in a TDMA manner in [213]. By contrast, in [217], all the UEs simultaneously upload their data to the BS using spatial division multiple access (SDMA). By extending the results of [213], Liu et al. [217] have considered a BS having multiple antennas and they have studied the throughput fairness for the multiple UEs supported by the SDMA protocol, which has higher spectrum efficiency than its TDMA counterpart. The minimum uplink throughput among all the UEs has been maximised by jointly optimising the time allocation for downlink and uplink transmissions with the aid of downlink energy beamforming and by beneficial power allocation for the UEs' uplink transmissions. In order to solve this optimisation problem, a two-stage algorithm has been developed. A pair of low-complexity suboptimal solutions has also be developed and compared to the optimal counterpart. Furthermore, Liu et al. [217] have maximised the weighted sum-rate of all UEs by jointly optimising the time and power allocation in order to achieve more balanced uplink transmission throughputs. In general, the UE closer to the BS has a better channel condition than that of the cell edge. The UE closer to the $\mathrm{BS}$ is exploited for relaying the data from its peer to the BS. However, in order to fulfil this relaying task, this UE may reserve a certain fraction of its own time resources and a specific portion of its energy harvested during the previous downlink WET, which may impair the performance of its own uplink WIT. A joint time- and power-allocation algorithm has been proposed in [217] for improving the achievable throughput in this cooperative system, while satisfying the required user fairness.

Yang et al. [219] have formulated the maximisation of the minimum uplink throughput among UEs for an IDEN system consisting of a BS having multiple antennas and multiple UEs having a single antenna. Both the BS and UEs operate in a time-division-duplex manner. Each transmission frame is constituted by three phases, namely the channel estimation phase, the downlink WET phase and the uplink WIT phase. Their numerical results have demonstrated that the required fairness among the UEs may be achieved by an asymptotic optimal solution.

Apart from the above-mentioned throughput fairness among UEs, energy fairness has also been considered by Zhang et al. 
[220], where the power splitting based receiver architecture of Fig.23 has been used by the UEs. The system studied consists of a multi-antenna transmitter and multiple receivers equipped with a single antenna. The authors have maximised the minimum energy harvested by all the receivers subject both to the minimum SINR requirement and to the maximum total transmit power. Unfortunately, the max-min problem of the energy harvested is not in a convex form, since the number of constraints is infinite. As a result, the original optimisation problem has to be firstly converted into an equivalent problem having a finite number of constraints. Then, a convex optimisation problem can be obtained and solved by exploiting the semi-definite relaxation (SDR) technique [220].

3) Energy Consumption Minimization: In order to create an environment-friendly IDEN system, another important objective is to minimise the energy dissipation of the IDEN system itself, while maintaining the QoS of the UEs' WET and WIT services.

The minimisation of the total transmit power of the BSs in IDEN systems, subject to the specific SINR requirements and received energy requirements have been studied in [221][225] for a MISO aided IDEN system supporting multiple UEs, where the transmitter is equipped with multiple antennas, while the UEs are quipped with a single antenna. The power splitting based receiver architecture has been conceived for integrated data and energy reception, as illustrated in Fig.23.

Shi et al. [221] have formulated the energy consumption minimisation as a non-convex problem. Then, the problem has been solved by exploiting the SDR technique and they have proved that their solution is optimal for the sake of minimising the total energy consumption. Furthermore, a pair of suboptimal algorithms, namely the zero-forcing based algorithm and the beamforming algorithm for optimising the SINR have been developed in order to reduce the computational complexity. Their numerical results have demonstrated that the performance of the proposed suboptimal solutions is close to the optimal one. Moreover, Liao et al. [222] have proposed a SDR aided randomisation approach for finding the upper-bound of their energy consumption minimisation problem. Compared to the solution of [221], the algorithm advocated in [222] significantly reduces the computational complexity, while achieves near-optimality in terms of the energy consumption minimisation.

Considering the practical implementation of resource allocation schemes, imperfect channel state information has been considered in [223] and [224]. Specifically, Wang et al. [223] have developed a pair of robust joint beamforming and power splitting schemes for minimising the energy consumption of the transmitter. In the first scheme, both the worst-case SINR and energy harvesting constraints have been taken into account. They have demonstrated that the worst-case of the joint beamforming and power splitting optimisation problem can be relaxed to a semi-definite programing (SDP) problem by equivalently transforming the linear matrix inequality based constraints to the robust quadratic matrix inequality based constraints. In their second scheme, the chance constraints ${ }^{8}$ for both the SINR and energy harvesting requirements have been considered. Hence, the chance constrained joint beamforming and power splitting problem has been transformed into another convex SDP problem by exploiting both the semi-definite relaxation and Bernstein-type inequality restriction. Furthermore, in [224], the effects of the imperfect channel estimation have been further considered. In the first case, the channel estimation error has been modelled by a Gaussian random variable, when the transmitter estimates the channel based on the UEs' uplink transmissions and the channel states are correlated. In the second case, all the estimated results of the channel covariance matrix have been modelled by independent Gaussian distributed random variables, which is justified by the fact that the estimation errors of covariance matrix are generated by multiple independent "error sources". Timotheou et al. [225] have explored the exploitation of the constructive interference in MISO downlink channels for improving the performance of both the information decoding and energy harvesting. They have also demonstrated that the constructive interference with the aid of beamforming can be exploited for improving the power level of the received RF signals, which may thus increase the amount of energy harvested.

Moreover, Cai et al. [226] have introduced multi-antenna aided relaying for improving the performance of integrated WET and WIT. Specifically, in order to minimise the total energy consumption of both the BS and the relay station, the classic alternating optimisation technique has been exploited for successively optimising the transceiver related parameters, including the BS's beamforming vectors, the relay station's amplify-and-forward transformation matrix and the power splitting factors at the single antenna aided receivers. Zhang et al. [227] have designed a secure beamforming scheme, which is capable of minimising the total transmit power, while maintaining the desired secrecy rate and the minimum requirement of the energy harvested. During the optimisation process, the transmit beamformer vector, the artificial noise vector and the power splitting factors are jointly determined. Furthermore, a two-stage algorithm has been developed for solving the associated non-convex energy consumption minimisation problem, based on which a near-optimal solution has been found by a low-complexity genetic algorithm.

Furthermore, in [228], the problem of minimizing the total transmit power has been studied by Demir et al. in a multigroup multicast based IDEN system, which is comprised of a multi-antenna aided BS and multiple UEs equipped with a single antenna. First, an efficient algorithm has been developed for antenna selection. It is demonstrated that the proposed algorithm outperforms the random antenna selection scheme. Then, a new hybrid beamforming structure has been introduced as an alternative to the antenna selection scheme. Finally, a pair of continuous-phase beamformers has been

\footnotetext{
${ }^{8}$ The chance-constrained method is one of the major approaches to solving optimization problems under various uncertainties. It is a formulation of an optimization problem that ensures that the probability of meeting a certain constraint is above a certain level. In other words, it restricts the feasible region so that the confidence level of the solution is high. - From https://optimization. mccormick.northwestern.edu/index.php/Chance-constraint_method
} 


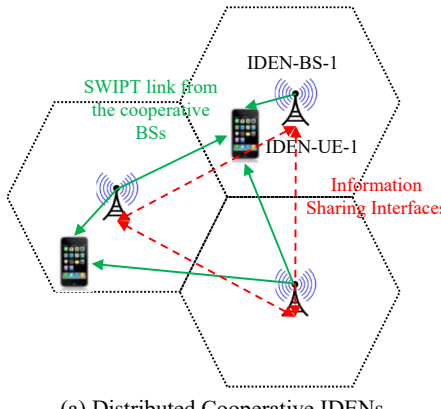

(a) Distributed Cooperative IDENs

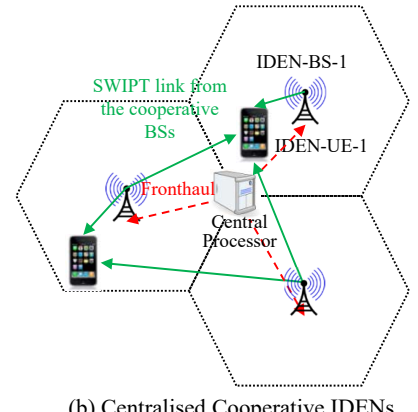

(b) Centralised Cooperative IDENs
Fig. 29. Cooperative IDENs

designed. In a nutshell, Table IX summarises some of the important contributions on the resource allocation issues of non-cooperative IDENs. Let us now consider the benefits of sophisticated cooperative schemes.

\section{B. Resource Allocation in Cooperative IDENs}

In cooperative IDENs, the UEs may not be specifically associated with a single BS but they can be connected to a specific group of the BSs, as shown in Fig.29. The cooperation of the BSs may provide substantial diversity and multiplexing gains, which thus significantly increases both the WET and WIT performance of the UEs. By contrast, in order to coordinating a number of the BSs for serving a single UE, these BSs have to be aware of all the channel state information as well as the information and energy requests from the UE. Exchanging this kind of information among the BSs imposes additional control signalling overhead on the dedicated information sharing links, as illustrated in Fig.29. The cooperative IDENs can be classified into the following categories:

- The Distributed Cooperative IDENs: As exemplified in Fig.29(a), the BSs are self-organised in an ad hoc manner for exchanging the channel state information, the data requested by the WIT users and the energy request of the WET users without the aid of a central processor. These BSs are connected with each other by either fibres or by wireless interfaces. For instance, in the current LTE cellular networks, the BSs are directly connected by the $\mathrm{X}_{2}$ interface for signalling exchange.

- The Centralised Cooperative IDENs: As exemplified in Fig.29(b), all the BSs are connected to a single central controller, which is capable of collectively coordinating all resources, such as time-, frequency-, power-, antennaresources for serving a UE's request for either WIT or for WET. A Cloud-Radio-Access-Network (C-RAN) is a typical centralised cooperative IDEN, where the cloud computing servers are clustered for collective signal processing and resource allocation.

1) Resource Allocation in Cooperative IDENs: A $K$-link MISO aided IDEN system, which consists of $K$ multi-antenna transmitters and $K$ single-antenna receivers, allows either the partial cooperation or the full cooperation of the transmitters. As for partial cooperation, the transmitters only have to exchange their channel state information with their peers so as to suppress the interference, while the data requested by a specific UE is only possessed by its associated transmitter. By contrast, for the full cooperation, the transmitters share both the channel state information and the data requested by a specific UE with their peers. As a result, beneficial diversity gains and multiplexing gains can be provided for substantially increasing the performance of the integrated WET and WIT. In this $K$-link MISO aided IDEN system, Xu et al. [229] have studied the throughout fairness by maximizing the minimum SINR of all the $K$ links. Additionally, in [230], [231] and [232], the energy consumption minimization of all the transmitters has been investigated. Coordinated multiplepoint (CoMP) cooperation has been considered as a key enabling technique in the forthcoming $5 \mathrm{G}$ era due to its capability of achieving energy-efficient communications and that of improving the cell-edge UE performance [233]. Moreover, Liang et al. [234] have also considered the throughput fairness in the coordinated multi-point (CoMP) aided IDEN system with full cooperation of the multiple transmitters. Zong et al. [235] have then extended the problem of energy consumption minimization from the single-antenna receivers to multi-antenna receivers.

$\mathrm{Xu}$ et al. [229] have studied the max-min SINR problem in the joint design of the transmit power allocations and the power splitting factors. The optimisation problem has been solved both in a centralised and in a distributed manner. The numerical results have demonstrated that the distributed algorithm is indeed optimal in termes of max-min SINR between the UEs in a two-UE case and both the centralised as well as the distributed algorithms are capable of improving the achievable max-min SINR subject to the specific amount of energy harvested. In [234], the minimum throughput among all the UEs have been maximised by Liang et al. subject both to the energy harvesting constraint of each receiver and to the transmit power constraint of each transmitter of the downlink transmissions of a CoMP aided IDEN system. This optimisation problem have been demonstrated to be NPhard. A combination of variable slacks, vectorisation, affine approximation and alternative convex optimisation (ACO) approaches have been exploited for transforming this NPhard problem into a convex one. Fortunately, the original NPhard optimisation problem can be decomposed into several convex sub-problems, which can be addressed by the classic second-order cone programming (SOCP) method. A heuristic algorithm has also been provided for solving the original NPhard problem.

In [230], three different approaches have been proposed by Zhao et al. for minimizing the total transmit power of all the transmitters. In the first approach, the original problem is relaxed by converting it into an SDP problem by exploiting the SDR technique. If a high-rank solution is derived, it would be converted to a feasible rank-one solution by exploiting the rank-one recovery method. In the second approach, the original problem has been formulated as an SOCP problem by exploiting both the SOCP relaxation and the Cauchy-Schwarz inequality. Compared to the SDRbased algorithm, the SOCP algorithm has a low computational 
TABLE IX

Summary of Resource Allocation in Non-cooperative IDENs

\begin{tabular}{|c|c|c|c|c|}
\hline Literature & Design objective & $\begin{array}{r}\text { Receiver } \\
\text { architecture }\end{array}$ & System model & CSI \\
\hline Ju et al. [211] & $\begin{array}{l}\text { Maximizing the } \\
\text { weighted sum-throughput }\end{array}$ & $\begin{array}{r}\text { TDMA: TS } \\
\text { OFDMA: PS }\end{array}$ & $\begin{array}{l}\text { Single-BS multiuser down- } \\
\text { link MISO SWIPT system }\end{array}$ & $\begin{array}{r}\text { Perfect } \\
\text { CSI }\end{array}$ \\
\hline Yang et al. [212] & Maximize the sum-throughput & SS & $\begin{array}{l}\text { Single-BS multiuser down- } \\
\text { link MISO SWIPT system }\end{array}$ & $\begin{array}{r}\text { Imperfect } \\
\text { CSI }\end{array}$ \\
\hline Zhang et al. [213] & $\begin{array}{r}\text { P1: Maximizing the sum- } \\
\text { throughput; P2: Maximizing } \\
\text { the minimum throughput }\end{array}$ & TS & $\begin{array}{r}\text { Single-BS } \\
\text { multiuser WPCNs }\end{array}$ & $\begin{array}{r}\text { Perfect } \\
\text { CSI }\end{array}$ \\
\hline Shi et al. [214] & $\begin{array}{l}\text { P1: maximizing the sum-throug- } \\
\text { hput; P2: Maximizing the total } \\
\text { the total energy harvested by all } \\
\text { the WET UEs; P3: Maximizing } \\
\text { the sum-throughput of all the } \\
\text { WIT UEs }\end{array}$ & $\begin{array}{l}\text { Separated } \\
\text { WET and } \\
\text { WIT UEs }\end{array}$ & $\begin{array}{l}\text { Single-BS multiuser down- } \\
\text { link MIMO SWIPT system }\end{array}$ & $\begin{array}{r}\text { Perfect } \\
\text { CSI }\end{array}$ \\
\hline Liao et al. [215] & $\begin{array}{l}\text { Maximizing both of the through- } \\
\text { puts of the WIT UEs and the en- } \\
\text { ergy harvested by the WET UEs. }\end{array}$ & $\begin{array}{l}\text { Separated } \\
\text { WET and } \\
\text { WIT UEs }\end{array}$ & $\begin{array}{l}\text { Single-BS multiuser down- } \\
\text { link MIMO SWIPT system }\end{array}$ & $\begin{array}{r}\text { Perfect } \\
\text { CSI }\end{array}$ \\
\hline $\begin{array}{l}\text { Wang et al. [217], } \\
\text { Timotheou et al. }[219]\end{array}$ & $\begin{array}{l}\text { Maximizing the minimum throu- } \\
\text { ghput among the UEs }\end{array}$ & TS & $\begin{array}{r}\text { Single-BS } \\
\text { multiuser WPCNs }\end{array}$ & $\begin{array}{r}\text { Perfect } \\
\text { CSI }\end{array}$ \\
\hline Zhu et al. [218] & $\begin{array}{r}\text { Maximizing the } \\
\text { weighted sum-throughput }\end{array}$ & TS & $\begin{array}{r}\text { Single-BS } \\
\text { two-user WPCNs }\end{array}$ & $\begin{array}{r}\text { Perfect } \\
\text { CSI }\end{array}$ \\
\hline Cai et al. [220] & $\begin{array}{l}\text { Maximizing the minimum energy } \\
\text { harvested among the WET UEs }\end{array}$ & PS & $\begin{array}{l}\text { Single-BS multiuser down- } \\
\text { link MISO SWIPT system }\end{array}$ & $\begin{array}{r}\text { Perfect } \\
\text { CSI }\end{array}$ \\
\hline Zhang et al. [221] & $\begin{array}{r}\text { Minimizing the total transmit } \\
\text { power of the BS }\end{array}$ & PS & $\begin{array}{l}\text { Single-BS multiuser down- } \\
\text { link MISO SWIPT system }\end{array}$ & $\begin{array}{r}\text { Perfect } \\
\text { CSI }\end{array}$ \\
\hline $\begin{array}{l}\text { Demir et al. }[222] \\
\text { Xu et al. }[223] \\
\text { Zhao et al. }[224]\end{array}$ & $\begin{array}{r}\text { Minimizing the total transmit } \\
\text { power of the BS }\end{array}$ & PS & $\begin{array}{l}\text { Single-BS multiuser down- } \\
\text { link MISO SWIPT system }\end{array}$ & $\begin{array}{r}\text { Imperfect } \\
\text { CSI }\end{array}$ \\
\hline Timotheou et al. [225] & $\begin{array}{l}\text { Minimizing the total transmit } \\
\text { power of the BS }\end{array}$ & PS & $\begin{array}{l}\text { Single-BS multiuser down- } \\
\text { link MISO SWIPT system }\end{array}$ & N.A. \\
\hline Zhu et al. [226] & $\begin{array}{l}\text { Minimizing the total transmit } \\
\text { power of the BS }\end{array}$ & PS & $\begin{array}{l}\text { Single-BS multiuser MISO } \\
\text { relaying SWIPT system }\end{array}$ & $\begin{array}{r}\text { Imperfect } \\
\text { CSI }\end{array}$ \\
\hline Liang et al. [227] & $\begin{array}{r}\text { Minimizing the total transmit } \\
\text { power of the BS }\end{array}$ & PS & $\begin{array}{l}\text { Single-BS multiuser down- } \\
\text { link MISO SWIPT system } \\
\text { with the secure requirement }\end{array}$ & $\begin{array}{r}\text { Perfect } \\
\text { CSI }\end{array}$ \\
\hline Zong et al. [228] & $\begin{array}{r}\text { Minimizing the total transmit } \\
\text { power of the BS }\end{array}$ & PS & $\begin{array}{r}\text { Single-BS multi-group } \\
\text { multicast SWIPT system }\end{array}$ & $\begin{array}{r}\text { Perfect } \\
\text { CSI }\end{array}$ \\
\hline
\end{tabular}

complexity, yet it has achieved a performance close to the full-complexity bound. In the third approach, an iterative algorithm has been developed, which is capable of achieving near-optimal performance in terms of the transmit power minimisation, if the SDR-based algorithm yields a higherrank solution. In [231], four different algorithms have been proposed by Timotheou et al., namely the maximum ratio transmission beamforming, the zero-forcing beamforming and the regularised zero-forcing beamforming as well as a hybrid scheme that combines the maximum ratio transmission and the zero-forcing beamforming. Similar to [223] and [224], Zhu et al. [232] have assumed the availability of the realistic imperfect CSI at the transmitters. The norm of the channel vectors' estimation error is bounded. The total transmit power minimisation problem has been efficiently solved by using the classic Lagrangian multiplier method and the SDR technique. Furthermore, in order to reduce the complexity, the original problem has been converted to a relaxed SDP problem based on loose bounds. Moreover, in [235], the minimisation of the total transmit power has been studied by Zong et al. in an IDEN system by considering the multi-UE MIMO interference channels, which consist of $K$ multi-antenna transmitters and $K$ multi-antenna receivers. Three different algorithms have been developed by considering the trade-off between the achievable performance and the computational complexity imposed. In the first algorithm, a SDR based alternating optimization between the transmit beamformer, the power splitting factors and the linear receive filter has been exploited for approaching the optimal solution. In the second algorithm, a low-complexity algorithm has been proposed by partially decoupling the joint optimisation. In the third algorithm, a closed-form solution has been derived by relying on transmitter-side zero-forcing.

2) Resource Allocation for C-RAN: As depicted in Fig.29(b), a C-RAN consists of a central processor and a set of low-cost remote radio heads (RRHs). In a C-RAN, the central processor performs the power-hungry and computationallyintensive baseband signal processing, while the RRHs are responsible for all RF operations, such as analog filtering and power amplification. The RRHs are distributed across the network and connected to the central processor via fronthaul links. In particular, a C-RAN is also capable of supporting multiple WIT and WET UEs. The integration of C-RAN and WET has been investigated in [236], [237], [238] and [239].

$\mathrm{Ng}$ et al. [236] have studied the minimisation of the total transmit power and the minimisation of the maximum individual capacity consumption among all the backhaul links, which are subject to the minimum required SINR used as a constraint imposed on the multiple WIT UEs and also subject 
to a minimum required energy reception constraint of multiple WET UEs. The optimisation problem has been formulated by the weighted sum of the total transmit power and the maximum capacity consumption among all the backhaul links, which has been demonstrated to be both non-convex and NP-hard. Ng et al. have reformulated the optimisation problem by replacing the non-convex objective by its convex hull, which leads to a low-complexity suboptimal solution.

In [237], the RRHs and the central processor are equipped with renewable energy harvesters and they are capable of sharing their energy with each other. Taking into account the limited backhaul capacity, the energy cooperation between RRHs and the imperfect CSI of the energy harvesting enabled receivers, the total transmit power of the C-RAN considered has been minimized by guaranteeing the UEs' QoS in the context of secure WIT and efficient WET. For solving this problem, $\mathrm{Ng}$ et al. [237] have developed an optimal iterative algorithm based on the generalized Benders decomposition [240] and they have also developed a low-complexity suboptimal scheme, which has provided a locally optimal solution.

Liang et al. [238] have provided a dual polarized antennabased signal splitting method on both the transmitter and receiver sides, where the signal is split into information decoding and energy harvesting streams and they are transmitted as well as received via the vertical and horizontal polarizations, respectively. The energy harvesting stream does not impose any interference on the information detection, which facilitates high-quality zero forcing beamforming for information detection. The problem is then formulated by maximising the sum-rate, while satisfying the per-UE received energy requirement, the per-backhaul-link capacity and the per-RRH transmit power constraints. Compared to the traditional power splitting based receiver architecture of Fig.23, the proposed method has eliminated the backhaul resource requirement and cancelled the interference imposed by the energy harvesting stream on the information detection, thanks to the exploitation of the dual polarised antennas. The information stream of the RF signal can be transmitted by a zero-forcing beamformer for increasing the achievable data rate without compromising with any energy requests of the UEs, thereby further reducing the hardware complexity.

In [239], a coordinated BS energy management scheme has been proposed for C-RANs. For the UEs' association with the BSs, both the UE-centric clustering and the BScentric clustering are considered. In the UE-centric clustering scenario, each UE selects a cluster of the BSs. By contrast, in the BS-centric clustering, the BSs are authorised to support an optimal number of UEs based on their energy budget. For both of these two scenarios, Ariffin et al. [239] have investigated the optimal trade-off among the cooperation links of the BSs, the overall energy consumption of the BSs and the energy purchased from the retailer, while satisfying the QoS requirements of WIT and WET. It has been demonstrated that their energy management scheme significantly improves the feasibility of full cooperation in C-RANs and that the $\mathrm{BS}$-centric clustering is more profitable in real-time energy balancing. In closing, Table $\mathrm{X}$ has summarised the latest contributions in resource allocation of cooperative IDENs.

\section{MAC Protocols in IDENs}

Apart from resource allocation in the centralised IDEN, the MAC protocol should also be carefully designed for the multiple access of the WET and the WIT UEs in a distributed IDEN for the sake of reducing the potential collisions and interference, when they compete for accessing the limited transmission medium.

Ramezani et al. [241] have provided a survey of MAC protocol design conceived for wireless sensor networks (WSNs) integrated with energy harvesting. In [242], the analysis and design of wireless sensor networks relying on energy harvesting aided devices have been addressed by focusing on conventional MAC protocols, namely on TDMA, on framedALOHA and on dynamic-framed-ALOPHA (DFA) and by accounting for a range of issues arising due to the implementation imperfections of energy harvesting. A novel metric, referred to as the delivery probability, has been defined for evaluating the capability of a MAC protocol to deliver the measurement of any sensor to the intended destination (or fusion center). The interplay between the delivery probability and the delivery latency has been analytically investigated by invoking Markov models. Naderi et al. [243] propose a MAC protocol by optimising WET with special emphasis on the sensor nodes, while minimizing the disruption of WIT. The authors have described the mechanism of their protocol by the following steps:

- Setting the maximum energy charging threshold;

- Activating specific transmitters based on their collective impact on the charging time;

- Requesting and granting WET services;

- Evaluating the respective priorities in terms of WIT and WET.

By implementing this protocol, the authors have observed upto a $300 \%$ network throughput improvement over the classic modified unslotted carrier-sensing-multiple-access (CSMA) based MAC protocol [243].

At the time of writing, the MAC protocols conceived for energy harvesting aided wireless sensor networks have also been widely investigated [244]-[246]. In [244], a coordinator assisted MAC protocol has been proposed for cloud based applications. Pillai et al. [245] have proposed an adaptive duty-cycle based MAC protocol, which relys on the IEEE 802.15.4 standard. This protocol has been designed by carefully considering both the power consumption of the sensor nodes during their operation and their battery discharge characteristics. Nguyen et al. [246] have designed an adaptive MAC protocol for balancing the attainable WET and WIT performance of an IEEE 802.15.4 standard based IoT network. An algorithm has been developed for minimising the network contention and maximising the energy harvested by UEs, which in turn improves both the energy efficiency and the network throughput.

In order to balance the achievable throughput among sensor nodes, Kim et al. [247] has proposed a MAC protocol for wireless sensor networks relying on the wireless energy harvesting, which is featured by the following two characteristics: 
TABLE $X$

Summary of Resource Allocation in Cooperative IDENs

\begin{tabular}{|c|c|c|c|c|}
\hline Literature & Design objective & $\begin{array}{l}\text { Receiver } \\
\text { architec. }\end{array}$ & System model & CSI \\
\hline Xu et al. [229] & $\begin{array}{l}\text { Maximizing the minimum } \\
\text { SINR among all the UEs }\end{array}$ & PS & $\begin{array}{r}K \text {-link Interference } \\
\text { MISO SWIPT system }\end{array}$ & $\begin{array}{r}\text { Perfect } \\
\text { CSI }\end{array}$ \\
\hline $\begin{array}{l}\text { Zhao et al. }[230] \\
\text { Zhu et al. }[232]\end{array}$ & $\begin{array}{l}\text { Minimizing the total transmit } \\
\text { power of all the BSs }\end{array}$ & PS & $\begin{array}{l}K \text {-link interference } \\
\text { MISO SWIPT system }\end{array}$ & $\begin{array}{r}\text { Imperfect } \\
\text { CSI }\end{array}$ \\
\hline $\begin{array}{l}\text { Timotheou } \\
\text { et al. }[231]\end{array}$ & $\begin{array}{l}\text { Minimizing the total transmit } \\
\text { power of all the BSs }\end{array}$ & PS & $\begin{array}{r}K \text {-link interference } \\
\text { MISO SWIPT system }\end{array}$ & $\begin{array}{r}\text { Perfect } \\
\text { CSI }\end{array}$ \\
\hline $\begin{array}{l}\text { Liang } \\
\text { et al. [234] }\end{array}$ & $\begin{array}{l}\text { Maximizing the minimum } \\
\text { downlink rate among the UEs }\end{array}$ & PS & $\begin{array}{l}\text { Downlink CoMP aided } \\
\text { MIMO SWIPT system }\end{array}$ & $\begin{array}{r}\text { Perfect } \\
\text { CSI }\end{array}$ \\
\hline $\begin{array}{l}\text { Zong } \\
\text { et al. [235] }\end{array}$ & $\begin{array}{l}\text { Minimizing the total transmit } \\
\text { power of all the BSs }\end{array}$ & PS & $\begin{array}{r}K \text {-link interference } \\
\text { MIMO SWIPT system }\end{array}$ & $\begin{array}{r}\text { Perfect } \\
\text { CSI }\end{array}$ \\
\hline $\begin{array}{l}\mathrm{Ng} \\
\text { et al. [236] }\end{array}$ & $\begin{array}{l}\text { Minimising the weighted sum } \\
\text { of the total transmit power and } \\
\text { the maximum individual back- } \\
\text { haul capacity consumption }\end{array}$ & $\begin{array}{l}\text { Separated } \\
\text { WET and } \\
\text { WIT UEs }\end{array}$ & $\begin{array}{r}\text { C-RAN or distributed } \\
\text { antenna system }\end{array}$ & $\begin{array}{r}\text { Perfect } \\
\text { CSI }\end{array}$ \\
\hline $\begin{array}{l}\mathrm{Ng} \\
\text { et al. [237] }\end{array}$ & $\begin{array}{l}\text { Minimizing the total transmit } \\
\text { power of all the RHHs }\end{array}$ & $\begin{array}{l}\text { Separated } \\
\text { WET and } \\
\text { WIT UEs }\end{array}$ & $\begin{array}{r}\text { C-RAN or distributed } \\
\text { antenna system }\end{array}$ & $\begin{array}{r}\text { Imperfect } \\
\text { CSI }\end{array}$ \\
\hline $\begin{array}{l}\text { Liang } \\
\text { et al. [238] }\end{array}$ & $\begin{array}{l}\text { Maximizing the sum-rate of the } \\
\text { UEs' downlink transmission }\end{array}$ & $\begin{array}{l}\text { Separated } \\
\text { WET and } \\
\text { WIT UEs }\end{array}$ & $\begin{array}{r}\text { C-RAN or distributed } \\
\text { antenna system }\end{array}$ & $\begin{array}{r}\text { Perfect } \\
\text { CSI }\end{array}$ \\
\hline $\begin{array}{l}\text { Ariffin } \\
\text { et al. [239] }\end{array}$ & $\begin{array}{l}\text { Optimising the trade-off among } \\
\text { the RHHs' cooperation links, } \\
\text { the total energy consumption } \\
\text { and the energy purchased }\end{array}$ & $\begin{array}{l}\text { Separated } \\
\text { WET and } \\
\text { WIT UEs }\end{array}$ & $\begin{array}{r}\text { C-RAN or distributed } \\
\text { antenna system }\end{array}$ & $\begin{array}{r}\text { Perfect } \\
\text { CSI }\end{array}$ \\
\hline
\end{tabular}

- Energy-adaptive duty cycle management that controls the duty cycle of sensor nodes according to their energy harvesting rates;

- Energy-adaptive contention algorithm that controls the contentions among sensor nodes by considering their WIT fairness.

In order to overcome the doubly near-far phenomenon defined in terms of both WET and WIT, Tamilarasi et al. [248] have proposed a new MAC protocol, which balances the attainable WIT and WET performance. The WIT of multiple sensor nodes requires the agreement of the wireless channel sharing. This protocol is capable of minimising the disruption of the WIT by optimising the WET provided for the sensor nodes.

Furthermore, the MAC protocol design has been considered not only for wireless sensor networks but also for other types of networks. Bedewy et al. [249] and Sami et al. [250] have proposed cooperative MAC protocols for energy harvesting aided cognitive radio networks (CRNs). In [251], an adaptive TDMA based MAC protocol has been designed by Qi et al. for energy harvesting aided wireless body area networks.

Specifically, in [250], a cooperative cognitive TDMA based MAC protocol has been proposed by carefully considering the QoS of primary networks. In the energy harvesting aided cognitive network considered, a certain portion of the licensed UEs' spectrum can be leased to unlicensed UEs. Hence, a higher probability of data transmission can be gained by the unlicensed UEs. Qi et al. [251] have designed an adaptive TDMA based MAC protocol for energy harvesting aided wireless body area networks, which aims for extending the network life time both by reducing the transmission latency and by increasing the efficiency of the channel utilisation. Additionally, a time slot allocation algorithm has been proposed for ensuring that the energy consumption is kept lower than the energy harvested by correspondingly adapting the duty cycle. Furthermore, this algorithm is also capable of improving the efficiency of the channel utilisation by avoiding the idle state of a specific channel. In conclusion, Table XI has summarised some of the seminal contributions on MAC protocol design in IDENs.

\section{Connecting WET and WIT UEs IN IDENs}

In contrast to the wired content delivery networks studied in [252]-[254], where all the data centres are supported by the power grid, communication nodes in IDENs are either supplied by stable energy sources or are capable of harvesting energy from the ambient environment. RF-signal based WET offers a flexible networking option for enabling energy cooperation among communication nodes in IDENs. The energy cooperation in IDENs aims for supporting abundant energy flows in areas, where intense communication operations are demanded. As a result, the energy distribution of IDENs can be optimised and the network lifetime is thus prolonged. Optimal energy distribution schemes, which may be realised by both WET and cable based energy transfer, are capable of efficiently improving the energy efficiency, the network throughput and of reducing the network outage of IDENs.

In this section, we will discuss the latest contribution of both distributed networking and centralised networking enabled IDENs. Furthermore, some other practical applications of IDENs will also be introduced.

\section{A. Distributed IDENs}

We first investigate the routing strategies and resource allocations schemes of distributed IDENs, where IDEN UEs are organised in an ad hoc manner, as exemplified in Fig.30. Moreover, the protocols conceived for improving the content 
TABLE XI

SuMmary of MAC Protocol IN IDENs

\begin{tabular}{|c|c|c|c|c|}
\hline Literature & Protocol & Network & Design Objective & Enabling Technique \\
\hline $\begin{array}{l}\text { Naderi } \\
\text { et al. [243] }\end{array}$ & RF-MAC & EH-WSN & $\begin{array}{l}\text { Maximising WET to the } \\
\text { sensors, while minimizing } \\
\text { the disruption of the WIT }\end{array}$ & $\begin{array}{l}\text { Evaluating the priorities of WET and } \\
\text { WIT; Activating the transmitters based } \\
\text { on their collective impact on the WET }\end{array}$ \\
\hline $\begin{array}{l}\text { Rao } \\
\text { et al. [245] }\end{array}$ & $\begin{array}{r}\text { Duty cycle } \\
\text { adapted MAC }\end{array}$ & EH-WSN & $\begin{array}{r}\text { Enhancing the network } \\
\text { lifetime }\end{array}$ & $\begin{array}{l}\text { Duty cycle adaptation by considering } \\
\text { the sensors' power consumption and } \\
\text { and their discharge characteristics; }\end{array}$ \\
\hline $\begin{array}{l}\text { Nguyen } \\
\text { et al. [246] }\end{array}$ & RF-AASP & EH-WSN & $\begin{array}{l}\text { Addressing the balance } \\
\text { between the energy } \\
\text { efficiency and the QoS }\end{array}$ & $\begin{array}{l}\text { Adapting the sleeping period in res- } \\
\text { ponse to the varying bursty traffic } \\
\text { load from the IoT applications }\end{array}$ \\
\hline $\begin{array}{l}\text { Kim } \\
\text { et al. [247] }\end{array}$ & $\begin{array}{r}\text { Energy } \\
\text { adapted MAC }\end{array}$ & EH-WSN & $\begin{array}{l}\text { Improving the fairness } \\
\text { of the achievable throu- } \\
\text { ghout among the sensors }\end{array}$ & $\begin{array}{r}\text { Adapt the sensors' duty cycles and } \\
\text { contentions according to their EH } \\
\text { rates and throughput fairness }\end{array}$ \\
\hline $\begin{array}{l}\text { Tamilarasi } \\
\text { et al. }[248]\end{array}$ & RF-MAC & WPCN & $\begin{array}{l}\text { Improving the fairness } \\
\text { of the achievable throu- } \\
\text { ghout among the sensors }\end{array}$ & $\begin{array}{l}\text { Predicting the energy that can be } \\
\text { harvested; Coordinating the spectrum } \\
\text { for satisfying both the WET and WIT }\end{array}$ \\
\hline $\begin{array}{l}\text { Sami } \\
\text { et al. [250] }\end{array}$ & CC-TDMA & EH-CRN & $\begin{array}{l}\text { Improving the throughput } \\
\text { and the packet drop rate for } \\
\text { both licensed/unlicensed UEs }\end{array}$ & $\begin{array}{l}\text { Exploiting spectrum leasing approaches } \\
\text { for satisfying the QoS of the licensed } \\
\text { UEs, while increasing the throughput } \\
\text { of the unlicensed UEs }\end{array}$ \\
\hline $\begin{array}{l}\text { Qi } \\
\text { et al. [251] }\end{array}$ & $\begin{array}{r}\text { Adaptive } \\
\text { TDMA } \\
\text { MAC }\end{array}$ & WBAN & $\begin{array}{l}\text { Reducing the transmission } \\
\text { latency; improving the } \\
\text { spectrum efficiency }\end{array}$ & $\begin{array}{l}\text { Time-slot allocation and duty cycle } \\
\text { adaptation by considering the energy } \\
\text { harvesting rate and the data traffic }\end{array}$ \\
\hline
\end{tabular}

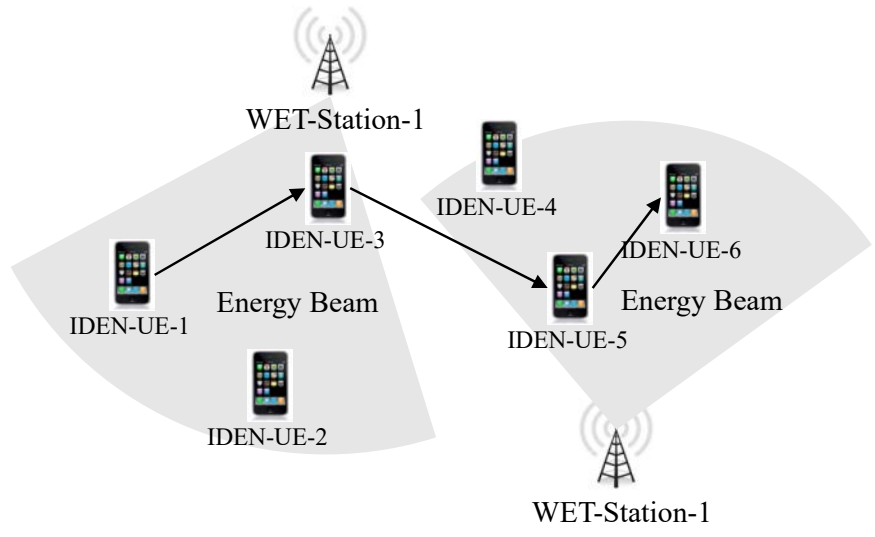

Fig. 30. An example of distributed IDEN

delivery are also discussed. Note that IDEN UEs are organised in an ad hoc manner, while their energy is supplied by the WET-Stations.

1) Routing strategy: As exemplified in Fig.30, an endto-end route is established for delivering the information from IDEN-UE-1 to IDEN-UE-6. IDEN-UE-3 is invoked for relaying the information, since it is capable of harvesting more RF energy from the WET-Station-1 than its counterpart IDEN-UE2. Although IDEN-UE-4 has a better link to IDEN-UE-3 than its counterpart IDEN-UE-5, we still select IDEN-UE-5 over it as the third relay, since IDEN-UE-5 is capable of harvesting more RF energy from WET-Station2 for forwarding information to the next hop. By jointly considering energy harvesting, the energy status, link qualities and network topology, a carefully-designed end-to-end routing scheme invoked for distributed IDENs is capable of reducing the energy consumption, of reducing the transmission latency and of increasing the throughput of multi-hop transmission. As a result, intensive efforts have been invested into routing design [255] [256] and transmission management [257] for diverse distributed IDENs.

Distributed networking enabled IDENs have been employed in a wireless sensor network consisting of energy harvesting communication nodes, as exemplified by a dynamic routing and operation strategy of the self-sustainable sensor networks proposed by Guo et al. [258], which significantly increase the network life-time.

An aggregate-and-send protocol has been proposed by Liu et al. [257] for the sake of balancing the energy consumption among the energy harvesting aided sensors in a wireless sensor network. In this protocol, by considering the energy status of the sensors, a number of packets are aggregated by the specific sensors having abundant energy in their batteries. The aggregated packets are then sent to the gateways. A pair of QoS-aware transmission schemes have been studied by Liu et al. [257]. The first one is the Markov decision based packets aggregation scheme. By considering the energy status of the sensors, this scheme decides the group of sensors in the duty of the packet aggregation and in the duty of the packet forwarding, while deciding number of packets to be aggregated and to be forwarded. Furthermore, a partial Kalman filter scheme with best-match aggregation has also been proposed by Liu et al. [257], which is capable of estimating the sensors' energy status with the aid of Kalman filtering. Furthermore, the number of packets to be aggregated and to be forwarded is decided by the best-matching procedure advocated.

Yao et al. [259] has introduced a delay-aware energy balancing scheme in order to minimise the average transmission delay. In their model, an adaptive harvest-then-cooperate protocol has been proposed, where the UEs firstly harvest energy from the APs and then send their data to the APs by establishing cooperation among their peers for the sake of overcoming the adverse effect of the unbalanced energy harvesting efficiency induced by the UEs' random locations. Additionally, a UE-scheduling scheme has been developed for 
this protocol in order to avoid data overflow at the UEs operating as relays. The average transmission delay is approximated by a Markov decision process and a low-complexity algorithm has been developed for obtaining a near-optimal solution. This protocol is capable of balancing the energy distribution, minimising the average transmission delay, whilst reducing packet loss.

Darji et al. [260] have stated that conventional routing protocols, such as LEACH [261], PEGASSI [262] and etc., are not suitable for energy harvesting enabled wireless sensor networks, since the sensors are only capable of supporting low-power operations. In this paper, a machine learning aided algorithm has been developed for minimising the networkwise energy consumption and thus maximising the network lifetime. Specifically, the clustering of the sensors in the wireless sensor network is completed by exploiting the classic genetic algorithm, which assists us in obtaining the optimal network lifetime.

Chin et al. [263] have studied a two-tier multi-hop network powered by the RF energy. This network consists of two sets of communication nodes, namely routers and energy harvesting nodes. The routers are continuously supplied by the stable energy sources, while they are also in duty of forwarding the data to the destinations. The energy emitted by the routers for the data forwarding may also be gleaned by the energy harvesting nodes. However, some routers are activated less often than others for the data forwarding. As a result, the energy harvesting nodes around these routers may not glean sufficient energy for satisfying their recharging requirement. Additional RF signals without carrying any data have to be emitted by these routers for charging these energy harvesting nodes, which might impose extra interference on the other routers' data reception. Therefore, by considering the interplay between the data transmission and the energy harvesting, Chin et al. have minimised the length of a super-frames, during which each router in the network may receive a certain portion of time for scheduling either their data forwarding tasks or their dedicated energy charging tasks.

Zhang et al. [264] have proposed a novel clustering based routing algorithm for the energy harvesting aided wireless sensor network, in which the sensors have to operate without stable energy supplies. In the clustering phase of the proposed routing algorithm, the network is partitioned into several clusters, while the cluster heads are elected according to their residual energy as well as their energy harvested. In the data transmission phase, a cluster head is capable of sending packets directly to the gateways, if its residual energy plus the energy harvested is higher than a certain threshold. Otherwise, the multi-hop routing have to be adopted among all the cluster heads for reducing the energy consumption of the data transmission.

The routing strategies in distributed IDENs have been summarised in Table XII

2) Resource allocation: In distributed IDENs, a large amount of tele-traffic has to be accommodated by efficiently coordinating precious communication resources. Resource allocation problems have been studied in numerous previous contributions in the context of different optimisation objec- tives, such as maximising the total throughput, minimising the energy consumption and etc..

Li et al. [265] have studied a heterogeneous ad hoc networks consisting of multiple transmitter and receiver pairs, where the transmitters are capable of harvesting renewable energy from the ambient environment and they are also capable of competing for the shared medium. Two different energy harvesting models are conceived, namely the constant energy harvesting rate and the stochastic but independently and identically distributed energy harvesting rate. A distributed opportunistic scheduling framework has been proposed for quantifying the role of both the energy state information and the channel state information. All the transmitters compete for accessing the channel in a random manner. If a transmitter successfully access the channel, it may make one of the following decisions based on its local energy state information and channel state information:

- Release the channel and restart the medium competition;

- Directly transmit its data until the end of this transmission block;

- Harvest energy at the beginning of the transmission block and send data during the rest.

Both the optimal throughput and scheduling schemes have been obtained by an appropriate searching technique. The proposed distributed opportunistic scheduling framework outperforms the best-effort delivery method in terms of the total throughput.

An optimal data gathering scheme has been proposed by Zhang et al. [266] for dynamic sensing and routing in the energy harvesting aided wireless sensor network. This scheme relies on sequentially running the following two algorithms. The first algorithm, named as the balanced energy allocation scheme, aims for minimising the total amount of the wasted harvested energy and minimising the variation of the energy allocation optimising the energy, which decides how much energy is consumed for the data sensing and how much energy is consumed for the data transmission, by considering both the sensors' battery levels as well as the amount of energy harvested. Given the derived energy allocation scheme, the second algorithm aims for maximising the total utility of the data sensing by optimising the distributed sensing rate and the routing control. Their simulation results are based on real experimental data and demonstrate the advantage of the proposed algorithms over a range of existing algorithms in terms of the data sensing performance.

Hentati et al. [267] has investigated the on-line maximisation of the total amount of the transmitted data by optimally designing the energy allocation scheme in an energy harvesting aided wireless sensor network. In their model, both the finite battery capacities and wireless fading channels have been taken into account. The energy allocation problem is formulated as a Markov decision process, which dynamically decides the ratio of the amount of energy allocated for the data sensing and that for the data transmission during a single time slot. Both a high-complexity optimal algorithm and a sub-optimal low-complexity algorithm are proposed for solving the optimisation problem. The simulation results have 
TABLE XII

SUMMARY OF THE ROUTING STRATEGIES IN DISTRIBUTED IDENS

\begin{tabular}{|l|r|r|r|}
\hline Literature & System model & Design goal & Proposed protocol \\
\hline $\begin{array}{l}\text { Liu } \\
\text { et } \text { al. } \text { [257] }\end{array}$ & $\begin{array}{r}\text { Energy harvesting } \\
\text { aided WSN }\end{array}$ & $\begin{array}{r}\text { Balancing the energy consumption } \\
\text { among the sensors }\end{array}$ & $\begin{array}{r}\text { Choosing the specific sensors for } \\
\text { packet aggregation and deciding } \\
\text { the number of packets to be sent }\end{array}$ \\
\hline $\begin{array}{l}\text { Yao } \\
\text { et al. } \text { [259] }\end{array}$ & $\begin{array}{r}\text { Wireless powered } \\
\text { cooperative network }\end{array}$ & $\begin{array}{r}\text { Minimizing the average transmiss- } \\
\text { ion delay, balancing the energy } \\
\text { consumption, reducing the packet loss }\end{array}$ & $\begin{array}{r}\text { Markov decision process aided delay } \\
\text { estimation, optimal cooperation est- } \\
\text { ablishment, harvest-and-cooperation }\end{array}$ \\
\hline $\begin{array}{l}\text { Darjit } \\
\text { et al. } \text { [260] }\end{array}$ & $\begin{array}{r}\text { Energy harvesting } \\
\text { aided WSN }\end{array}$ & $\begin{array}{r}\text { Minimizing the energy consumption } \\
\text { Maximising the network lifetime }\end{array}$ & $\begin{array}{r}\text { Genetic algorithm aided sensor } \\
\text { clustering }\end{array}$ \\
\hline $\begin{array}{l}\text { Chin } \\
\text { et al. } \text { [263] }\end{array}$ & $\begin{array}{r}\text { Two-tier Energy } \\
\text { harvesting multi- } \\
\text { hop network }\end{array}$ & $\begin{array}{r}\text { Minimizing the length of the super } \\
\text {-frame length and satisfying both } \\
\text { the data and energy requirements }\end{array}$ & $\begin{array}{r}\text { Joint routing and scheduling scheme } \\
\text { for the routers in the network }\end{array}$ \\
\hline $\begin{array}{l}\text { Zhang } \\
\text { et al. } \text { [264] }\end{array}$ & $\begin{array}{r}\text { Energy harvesting } \\
\text { aided WSN }\end{array}$ & $\begin{array}{r}\text { Addressing the unstable and uneven } \\
\text { energy distribution and reducing the } \\
\text { packet loss rate }\end{array}$ & $\begin{array}{r}\text { Partitioning the network into unequal } \\
\text { clusters and deciding the cluster heads } \\
\text { and routing data to the gateways }\end{array}$ \\
\hline
\end{tabular}

demonstrated that the proposed sub-optimal solution strikes an attractive performance versus complexity trade-off.

Lu et al. [268] have focused on the resource allocation of a heterogeneous cognitive radio aided energy harvesting sensor network. The energy harvesting enabled spectrum sensors in this network scan the licensed spectrum for detecting spectrum holes, while the battery-powered data sensors monitor and upload the sensing results in these detected spectrum holes. A resource allocation scheme consisting of a pair of sequential phases has been proposed by Lu et al. [268]. In the first phase, a spectrum sensor scheduling algorithm is conceived for maximising the time available for using the channels. In the second phase, a resource allocation scheme is carried out for minimising the energy consumption of all the data sensors by efficiently scheduling their uploading tasks on available resources in time-, power- and frequency- domains. The simulation results have demonstrated that the proposed resource allocation scheme is capable of achieving higher spectral and energy efficiencies.

The energy efficient device-to-device (D2D) communication [269] constitutes a pivotal element of the 5G architecture. D2D networks are capable of accommodating more simultaneous communication pairs, of increasing the network capacity and of improving the bandwidth and energy efficiency [270]. A social-ware energy harvesting assisted device-to-device architecture was proposed in [271].

Furthermore, the D2D communication is regarded as a key enabling technique of secure cyber-physical communications [272]. Specifically, Liu et al. [273] focused their attention on exploring the secure device-to-device communication in large-scale cognitive cellular networks, the transmitters harvest energy from the dedicated power beacons for supporting their data transmissions. Three beneficial WET policies have been introduced in their model, namely cooperative WET among the power beacons, the best power beacon aided WET and the nearest power beacon assisted WET. Furthermore, both an optimal and suboptimal receiver selection schemes have been proposed for the device-to-device based WIT. Closedform expressions are formulated for characterising the security level of the network in terms of the power outage probability, the secrecy outage probability and the secrecy throughput.

Saleem et al. [274] have embedded the solar energy har- vester into the devices for supporting their direct communication. Their optimisation problem aims for maximising the sumthroughput by optimally allocating the transmit power subject to the devices' residual energy and the energy harvested by their solar energy harvesters modules. An energy predictor, regarded as Pro-Energy [274], has been invoked for the sake of forecasting the energy arrival by exploiting the real solar energy harvesting data trace. They have thus demonstrated that their scheme is capable of substantially extending the network lifetime and of achieving the highest sum throughput.

Table XIII shows the seminal resource allocation contributions in distributed IDENs.

3) Delay tolerant content delivery: In a delay tolerant network, stable communication links are hard to maintain, which results in a long delay of data transmission. Endto-end content delivery in a delay tolerant network mainly relies on the multi-hop data forwarding among the mobile communication nodes.

Lu et al. [275] aimed for improving the performance of opportunistic forwarding in delay tolerant mobile networks relying on energy harvesting. The optimisation problem is formulated for maximising the end-to-end packet delivery ratio, which can be solved by exploiting the Markov decision process. During a single time slot, a packet carrier may decide either to consume energy for forwarding the packet immediately or to save energy for packet forwarding during the upcoming time slots. An adaptive $M$-step look-ahead algorithm has been developed for alleviating the computational complexity, where $M$ can be adaptively adjusted based on the currently available energy and on the energy that might be harvested in the future. The simulation results have demonstrated that the proposed scheme is capable of improving the energy efficiency, especially when the energy arrival rate is low.

Bouachir et al. [276] have proposed an opportunistic routing and data dissemination protocol for energy harvesting enabled wireless sensor network. In their model, synchronisation and coordination between the services in the application layer and the routing protocol in the network layer are allowed. Data has been classified and aggregated into two specific types: delay sensitive data and delay tolerant data. A packet holder may select potential relays from their neighbours based on a pair of considerations: the number of hops for creating 
TABLE XIII

SUMMARY OF RESOURCE ALLOCATION SCHEMES IN DISTRIBUTED IDENS

\begin{tabular}{|c|c|c|c|c|}
\hline Literature & System model & Design goal & Solution & CSI \\
\hline $\begin{array}{l}\mathrm{Li} \\
\text { et al. [265] }\end{array}$ & $\begin{array}{l}\text { Energy harvesting } \\
\text { aided ad hoc net. }\end{array}$ & $\begin{array}{l}\text { Maximising the } \\
\text { network throughput }\end{array}$ & $\begin{array}{r}\text { Two-stage energy probing scheme } \\
\text { and a distributed opportunistic } \\
\text { scheduling scheme }\end{array}$ & Perfect \\
\hline $\begin{array}{l}\text { Zhang } \\
\text { et al. }[266]\end{array}$ & $\begin{array}{r}\text { Energy harvesting } \\
\text { aided WSN }\end{array}$ & $\begin{array}{r}\text { Minimising the wasted harv- } \\
\text { ested energy and the variation } \\
\text { of the energy allocation; max- } \\
\text { imising the network utility of } \\
\text { the data sensing }\end{array}$ & $\begin{array}{l}\text { Balanced energy allocation scheme } \\
\text { and the optimal distributed sensing } \\
\text { rate and routing control }\end{array}$ & N.A. \\
\hline $\begin{array}{l}\text { Hentati } \\
\text { et al. }[267]\end{array}$ & $\begin{array}{r}\text { Energy harvesting } \\
\text { aided WSN }\end{array}$ & $\begin{array}{l}\text { Maximising the total amount } \\
\text { of the transmitted data }\end{array}$ & $\begin{array}{r}\text { Markov decision process aided } \\
\text { optimal energy allocation }\end{array}$ & Perfect \\
\hline $\begin{array}{l}\mathrm{Lu} \\
\text { et al. [268] }\end{array}$ & $\begin{array}{l}\text { Energy harvesting } \\
\text { aided CR-WSN }\end{array}$ & $\begin{array}{l}\text { Minimising the energy cons- } \\
\text { umption of the data sensors } \\
\text { Maximising the time available } \\
\text { for using the channels }\end{array}$ & $\begin{array}{r}\text { Scheduling the spectrum sensors } \\
\text { and allocating resources in time-, } \\
\text { power-, frequency-domain for the } \\
\text { data sensors }\end{array}$ & N.A. \\
\hline $\begin{array}{l}\text { Liu } \\
\text { et al. [273] }\end{array}$ & $\begin{array}{l}\text { Energy harvesting } \\
\text { aided D2D net. }\end{array}$ & $\begin{array}{r}\text { Maximizing the security level } \\
\text { in terms of the power outage, } \\
\text { secrecy outage and secrecy } \\
\text { throughput }\end{array}$ & $\begin{array}{l}\text { Cooperative WET among the po- } \\
\text { wer beacons, the best/nearest po- } \\
\text { wer beacon aided WET, optimal } \\
\text { receiver selection scheme }\end{array}$ & Perfect \\
\hline $\begin{array}{l}\text { Saleem } \\
\text { et al. } \text { [274] }\end{array}$ & $\begin{array}{l}\text { Energy harvesting } \\
\text { aided D2D net. }\end{array}$ & $\begin{array}{l}\text { Maximising the sum through- } \\
\text { put of the solar energy harve- } \\
\text { ster enabled D2D networks }\end{array}$ & $\begin{array}{l}\text { Optimally allocating the transmit } \\
\text { power by predicting the energy } \\
\text { arrival of the solar harvesters }\end{array}$ & Perfect \\
\hline
\end{tabular}

the forwarder list and the residual energy level of these neighbours. The simulation results have demonstrated that the proposed protocol exhibits a high reliability, scalability and energy exploitation efficiency.

Chen et al. [277] has proposed the harvest-then-cooperate protocol in a wireless-powered cooperative communication network, which consists of a hybrid access-point (AP), a source and a relay. The source and relay have no stable energy supply but they have capability of harvesting energy from the RF signals broadcasted by the AP. Based on this harvest-then-cooperate protocol, the source and relay firstly harvest the RF energy from the AP in the downlink and then cooperate to transmit information from the source to the AP in the uplink. The approximate close-form expression of the average throughput of the proposed two-hop network is established by taking the delay-limitation into consideration. Then, the analysis is extended to the realistic multi-relay scenario. There are two popular relay selection schemes, namely opportunistic relaying and partial relay selection, which are used for selecting the best relay to forward the packets. The throughput based on the above-mentioned pair of strategies has been compared and analysed. Based on the simulation results, the proposed protocol is compared to the existing harvest-thentransmit protocol and it is shown to have a better performance.

Mekikis et al. [278] have studied the connectivity of a wireless energy harvesting aided sensor network, in which the sensors in this network are batteryless and they can only glean energy from the dedicated power beacons. Furthermore, both the unicast and the broadcast based routing schemes are proposed both with and without the Rayleigh fading. After determining the probability of having an active sensor with the aid of the extensive Monte-Carlo simulation, the probability of connectivity for the pair of routing schemes can be obtained analytically. The simulation results provided compare the connectivity of the sensor network operating by relying on both batteryless and battery-powered sensors by using the pair of routing strategies advocated.

\section{B. Centralised IDENs}

Let us now focus our attention on the family of centralised networking enabled IDENs, which are exemplified in Fig.4. In this type of IDENs, the communication nodes are classified into the following categories:

- The Infrastructure includes the dedicated WET-BSs and WIT-BSs as well as the IDEN-BSs having the hybrid function of WET and WIT. Moreover, energy harvesting aided relay stations conceived for forwarding the requested information to the remote UEs also belong to the infrastructure. The infrastructure has a powerful signal processing capability and stable energy supply for radiating the RF signals.

- The User Equipments include various hand-held communication devices and low-power IoT devices, which cannot support complex signal processing tasks and they do not have sufficient energy supply for radiating the RF signals of high power density.

As a result, the centralised IDENs are capable of achieving a higher WET and WIT efficiency than their distributed counterparts, since both the optimal resource allocation scheme and the optimal route/relay selection scheme can be obtained by a powerful central processor. Furthermore, in the network planning stage, the density and the placement of the centralised infrastructure play essential roles in improving the WET and WIT performance.

Huang et al. [279] have deployed dedicated WET-BSs in cellular networks for charging the UEs via the RF signals. Hence the UEs become capable of supporting their uplink transmissions to the WIT-BSs. The tool of the classic stochastic geometry has been exploited to analytically study the coverage performance of this heterogeneous cell-based centralised IDEN in terms of both the power outage and communication outage, which are determined by diverse parameters, such as 
the density of the WET- and WIT-BSs, their transmit power etc..

To take a step further, Bi et al. [280] have focused on optimising the placement of the WET- and WIT-BSs, where the rechargeable UEs are capable of harvesting energy from the RF signals emitted by the dedicated WET BSs for supporting their own uplink transmissions. The placement optimisation scheme aims for minimising the number of the WET- and WIT-BSs by satisfying both the energy and data transmission requirements of the UEs. A pair of scenarios have been considered. In the first scenario, the WET- and WIT-BSs are geographically separated, while the WET and WIT BSs are co-located as IDEN-BSs in the second scenario. The proposed algorithm for solving the optimisation problem has been demonstrated to substantially reduce the number of WET and WIT BSs in the scenario of geographically separated WET- and WIT-BSs. By contrast, in the scenario that WET and WIT BSs are co-located, performance improvement of the IDEN-BSs is not significant.

Zhang et al. [281] investigated the energy efficiency of the cellular downlink relying on the WIT-BSs having stable energy supply and the energy harvesting aided relay stations. Due to the stochastic arrival of energy, the relay stations only remain active with a specific probability. Moreover, the relay stations were assumed to operate in a half-duplex mode and they adopt the classic decode-and-forward strategy for relaying data packets towards the destination. The relay stations are connected to the geographically closest WIT-BS and they all have a circular coverage with a radius of $R$. After obtaining the coverage probabilities of the relay aided downlink cellular network studied, the achievable average rate is used for quantifying both the spectral efficiency and the energy efficiency. The simulation results evaluate the influence of the WIT-BSs' and the relay stations' densities as well as the influence of the transmit power on the achievable average rate.

As for multi-cell networks, Nasir et al. [282] also proposed three resource allocation schemes relying on the WET aided relaying system, which have the objectives of sum-throughput maximisation, the minimum-throughput maximisation and the total power consumption minimisation, respectively. Both the geometric programming aided successive convex approximation method and difference-of-convex-function programming have been adopted for jointly optimising the transmit power of the IDEN-BSs and the relay stations as well as the signal splitting factors of the relay stations.

In mobile social networks, the social relationships of UEs are exploited for characterising the UEs' mobility patterns, which may further assists us in designing efficient data delivery schemes. Therefore, designing efficient communication schemes in the mobile social networks has to take the UEs' mobility patterns and their social relationships into account [283]. There are two main types of mobile social networks, namely the distributed mobile social networks and the centralised mobile social networks. In a distributed mobile social network, the UEs directly communicate with their peers, where the end-to-end routing scheme is essential for connecting a pair of UEs. By contrast, the UEs communicate with the infrastructure in a centralised mobile social network, where the placement of the infrastructure elements, such as BSs and WiFi access points, plays more important role in connecting the UEs.

Fan et al. [284] have studied the optimal deployment of the caching aided WIT access points provided for content delivery in a centralised mobile social network. Optimisation problems have been formulated for maximising the content delivery efficiency by jointly optimising the storage space allocation of the caching aided WIT access points and their placement. By further considering the UEs' interests in diverse locations, a linear programming model has been exploited for solving this optimisation problem. Furthermore, Fan et al. [285] have incorporated the WET function into the associated access points, which resulted in enhanced IDEN access points. The optimal deployment scheme of the IDEN access points has been obtained by exploiting the classic linear programming method for the sake of striking a balance between the UEs' WIT and WET requirements.

In conclusion, the latest contributions of the centralised IDENs have been summarised in TABLE XIV at a glance.

\section{Applications of IDENs}

Integrated WET and WIT has already been applied in some practical communication networks, such as industrial wireless sensor networks, vehicular networks and machine type communication aided IoTs.

The energy harvesting aided wireless sensor networks are widely applied for extending the network lifetime, reducing the energy consumption and recharging batteries of the industrial sensors [286]. Das et al. [287] have established a novel industrial wireless monitoring system, which has the ability of harvesting energy from the ambient environments. More applications of the energy harvesting aided wireless sensor networks have been introduced by Raj [288], such as greenhouse monitoring, vehicle detection and fire monitoring.

Berger et al. [289] have presented a robust low-power system relying on the energy harvesting and the appropriate energy management. A pair of energy harvesting chips, namely LTC3105 and TIBQ25504, associated with the same solar cell have been investigated for evaluating their performance of the energy management in different lighting scenarios. Their experiments have demonstrated that the maximum harvested energy can be achieved by the chip TIBQ25504 at the illumination of 750 Lux. By contrast, the maximum average harvested energy can be achieved by the chip LTC3105 at 1600 Lux. These test results provide a useful reference for the industrial application of the energy harvesting aided wireless sensor network.

Since the photovoltaic cells have been embedded into vehicles, the renewable energy can be harvested by supporting vehicle-to-vehicle communications. Design, implementation and optimisation of the energy harvesting assisted vehicular ad hoc networks have been studied by Ibrahim [290]. Furthermore, Atallah et al. [291] have summarised the latest contributions and envisioned potential challenges for energy harvesting assisted vehicular ad hoc networks. 
TABLE XIV

SUMMARY OF THE NETWORKING IN CENTRALISED IDENS

\begin{tabular}{|l|r|r|r|c|}
\hline Literature & System model & Design objective & Solution & CSI \\
\hline \hline $\begin{array}{l}\text { Huang } \\
\text { et al. } \text { [279] }\end{array}$ & $\begin{array}{r}\text { Het-Net consisting of } \\
\text { WET- and WIT-BSs. }\end{array}$ & $\begin{array}{r}\text { Coverage analysis in terms } \\
\text { of the power outage and } \\
\text { the communication outage }\end{array}$ & $\begin{array}{r}\text { Stochastic geometry, } \\
\text { Poisson point process }\end{array}$ & N.A. \\
\hline $\begin{array}{l}\text { Bi } \text { al. [280] } \\
\text { Het-Net consisting ei- } \\
\text { ther of WET- and WIT- } \\
\text { BSs or of IDEN-BSs }\end{array}$ & $\begin{array}{r}\text { Minimising the number of } \\
\text { WET- and WIT- BSs or } \\
\text { the number of IDEN-BSs }\end{array}$ & $\begin{array}{r}\text { Alternating method for the optimal } \\
\text { placement of WET- and WIT-BSs } \\
\text { and Greedy algorithm for the } \\
\text { optimal placement of IDEN-BSs }\end{array}$ & Perfect \\
et al. [281] & $\begin{array}{r}\text { Energy harvesting aided } \\
\text { Cooperative Networks }\end{array}$ & $\begin{array}{r}\text { Analysing the end-to-end } \\
\text { spectral efficiency and } \\
\text { energy efficiency }\end{array}$ & $\begin{array}{r}\text { Stochastic geometry, Poisson } \\
\text { point process and coverage } \\
\text { probability analysis }\end{array}$ & N.A. \\
\hline $\begin{array}{l}\text { Nasir } \\
\text { et al. } \text { [282] }\end{array}$ & $\begin{array}{r}\text { WET aided multi-cell } \\
\text { relaying system }\end{array}$ & $\begin{array}{r}\text { Maximising the sum- and } \\
\text { the minimum-throughput, } \\
\text { minimising the total power }\end{array}$ & $\begin{array}{r}\text { Jointly optimising the transmit po- } \\
\text { wer and signal splitting factors wi- } \\
\text { th the aid of geometric programm- } \\
\text { ing, difference-of-convex-function } \\
\text { programming. }\end{array}$ & Perfect \\
\hline $\begin{array}{l}\text { Fan } \text { et al. } \\
\text { [284] [285] }\end{array}$ & $\begin{array}{r}\text { Centralised mobile soci- } \\
\text { al network of integrated } \\
\text { WET and WIT }\end{array}$ & $\begin{array}{r}\text { Striking a balance between } \\
\text { the achievable sum-throughput } \\
\text { and the sum-energy harvested }\end{array}$ & $\begin{array}{r}\text { Deployment and the storage space } \\
\text { allocation of the IDEN access po- } \\
\text { ints by considering the UEs' social } \\
\text { interests and the mobility patterns }\end{array}$ & N.A. \\
\hline
\end{tabular}

Both the energy harvesting and the WET techniques have been integrated in the machine-type communications aided Internet of Things system, which results in that the legacy protocols are no longer directly applicable. As a result, novel protocols have been proposed by Alonso et al. [292] for both the MAC layer and network layer.

Rinne et al. [293] have explored the fundamental performance limits of the energy harvesting aided machine-tomachine communication system. The theoretical bound of the achievable throughput has been obtained by exploiting the classic Shannon's capacity and the power-law based path-loss model and by considering non-ideal communication links as well as the energy harvesting and storage capabilities. Their numerical results have characterised the feasibility regions of the proposed energy harvesting aided machine-to-machine system, which operates in the licence-exempt ISM bands at $433 \mathrm{MHz}$ and $900 \mathrm{MHz}$.

Furthermore, Xiao et al. [294] have studied a WET aided full-duplex machine-to-machine communication system, where the transmitter sends its information to the receiver, while the receiver simultaneously transfers its spare energy to the transmitter over the same frequency. A stochastic machineto-machine game has been exploited by Xiao et al. for characterising the interaction between the transmitter and the receiver. As a result, the maximum lifetime of the WET aided full-duplex machine-to-machine system has been achieved in a distributed manner without any assistance of centralised controller.

\section{Other Open Challenges}

Apart from the above subjects, there are numerous open challenges in the research of IDENs, which are briefly summarised in this section.

\section{A. Efficiency Enhancement of WET}

Although RF signals are capable of carrying remarkable energy to far-field locations, the WET efficiency remains low due to the serious channel attenuation incurred by pathloss, shadowing and multipath fading. As a result, a jointly optimised design has to be carried out by considering the entire WET chain, including the DC-RF energy converter at the transmitter, transmit/receive antenna design, the RF signal propagation over wireless channels and the RF-DC energy converter at the receiver. For instance, solid-state distributed RF amplifiers have to be invoked for the sake of increasing the DC-RF energy conversion efficiency at the transmitters. Beamforming relying on holographic antennas should also be implemented in order to counteract the serious channel attenuation. Furthermore, non-diffraction beams are capable of reducing the energy loss during the RF signals' propagation. Therefore, exploiting non-diffraction beams may be capable of remarkably increasing both the WET and the WIT performance. Additionally, the powerful meta-surface based energy harvesting technique should be implemented at the receivers in order to efficiently glean energy from the received RF signals, while advanced RF-DC circuits and battery charging circuits also have to be conceived.

Furthermore, the mathematical model of evaluating the efficiency of the entire WET chain remains an open problem in the literature, which requires joint efforts from both the electronic and communication engineers. This model may also be relied upon for jointly optimising all the components in a WET chain.

\section{B. Efficient Energy Storage}

In IDENs, the heterogeneous UEs may have different energy requirements, similar to their WIT counterparts. For example, some UEs require fast charging, which can be satisfied by establishing narrow energy beams gleaned from the energy beacons. Therefore, extremely high energy bursts may be delivered to the UEs in a very short instant. In order to accommodate these energy peaks, we have to focus our attention on the design of energy storage and management schemes from the perspective of battery energy density. Furthermore, more efforts should also be invested in charging/discharging 
management, energy balancing management and thermal management.

In IDENs, some UEs only require micro-level energy for supporting their low-power operations, which can be satisfied by establishing wide energy beams spanning from the energy beacons to the multiple energy requesters. Since the energy is distributed across a wide area, only a small fraction of the energy can be delivered to a single energy requester. Sometimes, the energy carried by the RF signals is too low to efficiently charge batteries. As a result, advanced voltage multiplier techniques have to be explored in order to efficiently charge the batteries with micro-level energy. Additionally, the management system required for the collection and storage of low-level energy all requires further attention, as an important part of battery charging techniques.

\section{Heterogeneous Internet of Energy}

Integrating all sorts of WET techniques together, including both near-field and far-field ones, may indeed satisfy the energy requirements of UEs. However, as stated in Section I, different WET techniques exhibit different charging ranges and charging efficiencies. As a result, coordinating these distinctive WET techniques and exploiting their cooperation is essential for further increasing the attainable WET efficiency. By considering the specific energy levels of the sources and the energy requirements of the requesters, both the energy beacons centralised and the UEs centralised networking and association techniques can be investigated by borrowing the classic stochastic geometry theory from the research of established wireless communication.

Furthermore, upon considering the distinctive features of WET, we believe that a maximum of two-hop WET can be realised for coordinating the energy flows in the heterogeneous Internet of Energy. For example, laser based WET is capable of realising high-efficiency point-to-point energy transmission. An energy relay station is capable of receiving a substantial amount of energy carried by laser. This energy may be further multicast to low-power devices in a wide RF beam. As a result, the energy relay stations have to be equipped with heterogeneous energy transceiver equipments. Furthermore, mobile energy stations can also be invoked for delivering energy from the source to the destinations. Fully exploiting the features of different WET techniques is capable of significantly extending the coverage of the Internet of Energy.

Moreover, efficient communication networking is also required for pilot exchange between the energy beacons and the energy requesters in order to promptly establish energy links for WET. As a result, the implementation of heterogeneous Internet of Energy has to rely on the integration with WIT, which augments the significance of IDEN again.

\section{Information Theoretic WET Capacity}

As stated in Section III, the rate-energy function is obtained by considering the energy required by the receivers as constraints in order to maximise the mutual information between the transmitters and receivers. This methodology inherently regards WET as a by-product of conventional WIT in IDENs.
However, both WET and WIT in IDENs should be fairly considered. As a result, designers of IDENs are also interested in the performance limits of WET in IDENs. Therefore, by following a similar methodology to that of Section III, we can change the objective function to the maximisation of the energy arriving at the receivers, which is subject to the mutual information requirement. Furthermore, the methodology can be extended for studying the performance limits of the integrated data and energy transfer in broadcast channels, in MIMO channels and in interference channels, just to name a few.

\section{E. Modulation Design}

In the 5G era, non-orthogonal-multiple-access (NOMA) is a key technique of increasing the bandwidth efficiencies and hence of the transmission rates. However, due to its non-orthogonal nature, the information requested by different UEs is superimposed. After passing through the channel, the information has to be detected and decoded sequentially by multiple UEs. However, when the information bits requested by multiple UEs are modulated independently at the downlink transmitter, the resultant RF signals may have different amplitudes and different phases. When these RF signal waves are superimposed during their transmissions, the amplitude of the superimposed signal is weakened if its components have opposite phases, while it is strengthened if its components have the same phases. However, from the perspective of WET, the superimposed signal having a higher amplitude is capable of delivering more energy to the UEs. As a result, the information bits from all NOMA UEs have to be jointly modulated for the sake of maximising the energy carried by the superimposed signal, while still satisfying the bit-error-ratio requirements.

In order to reduce the hardware complexity of IDEN receivers, rectifiers may be invoked both for converting the RF energy to the DC energy and for down-converting the RF signals from passband to baseband. However, only the amplitude information can be retained after the conversion action of rectifiers, while the phase and frequency information is lost. As a result, we should suitably redesign the modulation schemes for low-complexity IDEN receivers only having rectifiers by modulating information onto the amplitude of the signals. For example, pulse position modulation, pulse amplitude modulation, pulse width modulation and pulse interval modulation are potential candidates for integrated data and energy transfer.

\section{F. Interference Cancellation and Signal Decoupling}

As stated in Section I, WET and WIT requires different levels of input power in order to activate the energy reception circuits and the information reception circuits. The substantial gap between the input power requirements imposes challenges on the integrated data and energy transfer. IDEN stations are equipped with both functions of WET and WIT. IDEN UEs may receive both energy and data from same sources. In order to counteract the adverse effects of path-loss and hence to increase amount of energy received, in one hand, IDEN stations have to increase their transmit power, or the IDEN UEs should be closer to the IDEN stations. If IDEN UEs are 
distant from the IDEN stations, the RF signals arrive at a low power. These low-power RF signals can only be exploited for data reception, but they are not capable of activating the energy reception circuits. In a nutshell, IDEN UEs are only capable of receiving energy in the near-field of IDEN stations. By contrast, when IDEN UEs are in the far-field of IDEN stations, they may only receive data. This is the near-far effect in IDENs.

A potential solution for overcoming the near-far effect is to deploy separated dedicated WET and WIT infrastructure, respectively. Specifically, WET stations should have a high transmit power and they should be densely deployed within the proximity of IDEN UEs in order to reduce the energy loss incurred by path-loss. By contrast, WIT stations could be deployed in the far-field. Due to the channel attenuation, the received power of the RF signals carrying data is much lower than that carrying energy. The RF signals dedicated to WET may impose destructive interference on those dedicated to WIT. As a result, further efforts should be invested in improving interference cancellation and signal decoupling.

\section{G. Multiple Objectives based Resource Allocation}

As stated in Section IV, the existing contributions on IDENs mostly focus on resource allocation in an isolated domain for the sake of optimising a single-component objective. However, the available resources are in multiple domains, such as time, frequency, code, power, user and spatial domains. It may significantly increase the performance of integrated WET and WIT, if we deeply understand the mutual interactions among the resources in the different domains and develop joint resource scheduling schemes, which requires high-dimensional resource allocation schemes in IDENs. Furthermore, most of the current resource allocation schemes are obtained by optimising either a single WET or WIT performance indicator, while satisfying the other's minimum requirement. However, both WET and WIT should be equally treated in IDENs. As a result, a single-objective based optimisation is not appropriate. Pareto front based optimisation [295] can be relied upon for achieving the joint optimality of both WET and WIT, which deserves our further effort invested in multiple objective based optimisation algorithms and their applications in highdimensional resource allocation, as exemplified in the WIT domain by [295].

\section{H. Joint Data and Energy Routing}

Conventional routing designs in ad hoc networks typically assume that the communication nodes have stable energy sources. As a result, an end-to-end route is designed by only considering the communication channel qualities among the communication nodes of the network. However, this is not the case in the IoT, where the communication tasks of the IoT devices are usually supported by batteries. As a result, when an end-to-end route is designed, the battery status of IoT devices has to be taken into account. The IoT devices having low energy levels should not be frequently relied upon for routing data in order to extend the life-span of sensor networks. Furthermore, IoT devices can be supported by harvesting renewable energy. Devices having sufficient energy may share their energy with their peers by the transmission of RF signals. Consequently, apart from the battery status, we may also rely upon a IoT device operating as a relay, which is surrounded by other IoT devices having sufficient energy in their batteries. Although the relay may not have enough energy to forward data, other peers within its proximity may share their energy by radiating RF signals towards this relay for the sake of assisting its data forwarding operation. As a result, the data route and energy route should be jointly designed for ensuring that energy always flows to the specific node where it is required.

\section{Socially Aware Placement of IDEN Stations}

In practical scenarios, IDEN UEs are unevenly distributed. They have their own favourite places to visit and some places of interest may attract many IDEN UEs. Relying on stateof-the-art localisation techniques [296], [297], the mobility traces of the UEs can be acquired by the network operator. As a result, we have to firstly understand the UEs' geographic preferences, when we deploy IDEN in a specific area. We may model the UEs' movement according to their geographic preferences and study the steady-state probability of UEs being at a specific position. Different deployment schemes of IDEN stations may be designed for serving different purposes. For example, deployment schemes should be designed for maximising the successful data delivery rate, or for maximising the energy reception rate. Since both data and energy are transferred by RF signals, we should carefully design our deployment schemes for balancing both the WET and WIT.

\section{J. IDEN Aided Mobile Cloud Computing}

At the time of writing, big data enabled cellular networking is attracting much attention in the communication community [298]. Processing data in the cloud servers in an energyefficient manner is essential for sustainability [299]. Due to its flexibility and robustness, cloud computing has already been invoked for improving the wireless communication performance attained [300]. Furthermore, mobile cloud computing can also be integrated with our IDENs in order to support effective computation in low-power and low-complexity IoT devices. Specifically, an IDEN station may transfer energy to IoT devices for the sake of supporting their local computation or for supporting the computation to be offloaded to a node having better computing resources. Moreover, an IDEN station is also capable of offloading computing tasks from IoT devices to the mobile cloud. A set of policies has to be optimised for energy-efficient computing. First, the computing policy has to be optimised concerning whether the IoT devices should offload their computing tasks to the cloud or they should locally process them. Secondly, the IoT devices should optimally choose the time sharing between the WET phase and the computing and offloading phases so that the IoT devices may receive sufficient energy for supporting their subsequent operating phases. Furthermore, both the CPU-cycle statistics and the channel state information should be taken into account for maximising the computing related performance. 


\section{Conclusions}

In order to address the energy shortage of miniature communication devices in the future 5G and IoT era, engineers initially seek additional energy from renewable source. However, harvesting renewable energy heavily depends on the ambient environment, which results in its uncontrollability, unpredictability and unreliability. Therefore, we may opt for RF signal based WET as an alternative technique for supplying controllable and reliable energy to low-power communication devices and for realising a promising vision of charging anytime, anywhere. However, operating in the same spectral band may result in contradiction between RF based WET and conventional WIT. Understanding the inherent relationship between WET and WIT and hence efficiently coordinating both energy and information transfer in the RF spectral band results in the promising research topic of IDENs. This treatise provided the first detailed survey on the key techniques of IDENs from its bottom to top layers, including the information theoretical foundations of integrated WET and WIT in the same spectrum, the hardware implementation of the entire WET chain, the transceiver design of the physical layer, resource allocation and protocol design for MAC and networking techniques for connecting data and energy users in IDENs. However, numerous unsolved open challenges have to be overcome for further pushing IDEN from theory to practice. This treatise aims for providing insights into the emerging topic of IDENs, which may be used as a guidance for both engineers and researchers, who are willing to contribute to realising this promising vision.

\section{REFERENCES}

[1] F. Han, S. Zhao, L. Zhang, and J. Wu, "Survey of strategies for switching off base stations in heterogeneous networks for greener $5 \mathrm{~g}$ systems," IEEE Access, vol. 4, pp. 4959-4973, August 2016.

[2] A. Zanella, N. Bui, A. Castellani, L. Vangelista, and M. Zorzi, "Internet of Things for Smart Cities," IEEE Internet of Things Journal, vol. 1, no. 1, pp. 22-32, February 2014.

[3] A. Alrawais, A. Alhothaily, C. Hu, and X. Cheng, "Fog Computing for the Internet of Things: Security and Privacy Issues," IEEE Internet Computing, vol. 21, no. 2, pp. 34-42, March 2017.

[4] K. Wang, Y. Wang, Y. Sun, S. Guo, and J. Wu, "Green industrial internet of things architecture: An energy-efficient perspective," IEEE Communications Magazine, vol. 54, no. 12, pp. 48-54, December 2016.

[5] L. Chiaraviglio, N. Blefari-Melazzi, W. Liu, J. A. Gutierrez, J. van de Beek, R. Birke, L. Chen, F. Idzikowski, D. Kilper, P. Monti, A. Bagula, and $\mathrm{J}$. Wu, "Bringing $5 \mathrm{~g}$ into rural and low-income areas: Is it feasible?" IEEE Communications Standards Magazine, vol. 1, no. 3, pp. 50-57, September 2017.

[6] Y. Chen, N. Chiotellis, L. X. Chuo, C. Pfeiffer, Y. Shi, R. G. Dreslinski, A. Grbic, T. Mudge, D. D. Wentzloff, D. Blaauw, and H. S. Kim, "Energy-Autonomous Wireless Communication for Millimeter-Scale Internet-of-Things Sensor Nodes," IEEE Journal on Selected Areas in Communications, vol. 34, no. 12, pp. 3962-3977, December 2016.

[7] R. Atallah, C. Assi, and J. Y. Yu, "A Reinforcement Learning Technique for Optimizing Downlink Scheduling in an Energy-Limited Vehicular Network," IEEE Transactions on Vehicular Technology, vol. PP, no. 99, pp. 1-1, 2016.

[8] M. Liserre, T. Sauter, and J. Y. Hung, "Future Energy Systems: Integrating Renewable Energy Sources into the Smart Power Grid Through Industrial Electronics," IEEE Industrial Electronics Magazine, vol. 4, no. 1, pp. 18-37, March 2010.

[9] O. Ozel and S. Ulukus, "Achieving AWGN Capacity Under Stochastic Energy Harvesting," IEEE Transactions on Information Theory, vol. 58, no. 10, pp. 6471-6483, October 2012.
[10] O. Ozel, K. Tutuncuoglu, S. Ulukus, and A. Yener, "Fundamental limits of energy harvesting communications," IEEE Communications Magazine, vol. 53, no. 4, pp. 126-132, April 2015.

[11] Y. Dong, F. Farnia, and A. Özgür, "Near Optimal Energy Control and Approximate Capacity of Energy Harvesting Communication," IEEE Journal on Selected Areas in Communications, vol. 33, no. 3, pp. 540557, March 2015.

[12] S. Gupta, R. Zhang, and L. Hanzo, "Throughput Maximization for a Buffer-Aided Successive Relaying Network Employing Energy Harvesting," IEEE Transactions on Vehicular Technology, vol. 65, no. 8, pp. 6758-6765, August 2016.

[13] D. Shaviv, P. M. Nguyen, and A. Özgür, "Capacity of the EnergyHarvesting Channel With a Finite Battery," IEEE Transactions on Information Theory, vol. 62, no. 11, pp. 6436-6458, November 2016.

[14] C. Huang, R. Zhang, and S. Cui, "Optimal Power Allocation for Outage Probability Minimization in Fading Channels with Energy Harvesting Constraints," IEEE Transactions on Wireless Communications, vol. 13, no. 2, pp. 1074-1087, February 2014.

[15] R. Vaze, R. Garg, and N. Pathak, "Dynamic Power Allocation for Maximizing Throughput in Energy-Harvesting Communication System," IEEE/ACM Transactions on Networking, vol. 22, no. 5, pp. 1621-1630, October 2014

[16] F. Yuan, Q. T. Zhang, S. Jin, and H. Zhu, "Optimal Harvest-Use-Store Strategy for Energy Harvesting Wireless Systems," IEEE Transactions on Wireless Communications, vol. 14, no. 2, pp. 698-710, February 2015.

[17] S. Satpathi, R. Nagda, and R. Vaze, "Optimal Offline and Competitive Online Strategies for Transmitter-Receiver Energy Harvesting," IEEE Transactions on Information Theory, vol. 62, no. 8, pp. 4674-4695, August 2016.

[18] M. Rezaee, M. Mirmohseni, and M. R. Aref, "Energy Harvesting Systems With Continuous Energy and Data Arrivals: The Optimal Offline and Heuristic Online Algorithms," IEEE Journal on Selected Areas in Communications, vol. 34, no. 12, pp. 3739-3753, December 2016.

[19] W. Ni and X. Dong, "Energy Harvesting Wireless Communications With Energy Cooperation Between Transmitter and Receiver," IEEE Transactions on Communications, vol. 63, no. 4, pp. 1457-1469, April 2015.

[20] K. Tutuncuoglu and A. Yener, "Energy Harvesting Networks With Energy Cooperation: Procrastinating Policies," IEEE Transactions on Communications, vol. 63, no. 11, pp. 4525-4538, November 2015.

[21] Z. Chen, Y. Dong, P. Fan, and K. B. Letaief, "Optimal Throughput for Two-Way Relaying: Energy Harvesting and Energy Co-Operation," IEEE Journal on Selected Areas in Communications, vol. 34, no. 5, pp. 1448-1462, May 2016.

[22] R. Ramamonjison and V. K. Bhargava, "Energy Allocation and Cooperation for Energy-Efficient Wireless Two-Tier Networks," IEEE Transactions on Wireless Communications, vol. 15, no. 9, pp. 64346448, September 2016.

[23] N. Reyhanian, B. Maham, V. Shah-Mansouri, W. Tusher, and C. Yuen, "Game-Theoretic Approaches for Energy Cooperation in Energy Harvesting Small Cell Networks," IEEE Transactions on Vehicular Technology, vol. PP, no. 99, pp. 1-1, 2017.

[24] K. Hamedani, L. Liu, R. Atat, J. Wu, and Y. Yi, "Reservoir computing meets smart grids: Attack detection using delayed feedback networks," IEEE Transactions on Industrial Informatics, vol. 14, no. 2, pp. 734 743, February 2018.

[25] T. Han and N. Ansari, "A Traffic Load Balancing Framework for Software-Defined Radio Access Networks Powered by Hybrid Energy Sources," IEEE/ACM Transactions on Networking, vol. 24, no. 2, pp. 1038-1051, April 2016.

[26] Y. H. Chiang and W. Liao, "Green Multicell Cooperation in Heterogeneous Networks With Hybrid Energy Sources," IEEE Transactions on Wireless Communications, vol. 15, no. 12, pp. 7911-7925, December 2016.

[27] M. J. Farooq, H. Ghazzai, A. Kadri, H. ElSawy, and M. S. Alouini, "A Hybrid Energy Sharing Framework for Green Cellular Networks," IEEE Transactions on Communications, vol. 65, no. 2, pp. 918-934, February 2017.

[28] M. Sheng, D. Zhai, X. Wang, Y. Li, Y. Shi, and J. Li, "Intelligent Energy and Traffic Coordination for Green Cellular Networks With Hybrid Energy Supply," IEEE Transactions on Vehicular Technology, vol. 66, no. 2, pp. 1631-1646, February 2017.

[29] V. Chamola, B. Sikdar, and B. Krishnamachari, "Delay Aware Resource Management for Grid Energy Savings in Green Cellular Base Stations 
With Hybrid Power Supplies," IEEE Transactions on Communications, vol. 65, no. 3, pp. 1092-1104, March 2017.

[30] Q. Wu, M. Tao, D. W. K. Ng, W. Chen, and R. Schober, "EnergyEfficient Resource Allocation for Wireless Powered Communication Networks," IEEE Transactions on Wireless Communications, vol. 15, no. 3, pp. 2312-2327, March 2016.

[31] K. W. Choi and D. I. Kim, "Stochastic Optimal Control for Wireless Powered Communication Networks," IEEE Transactions on Wireless Communications, vol. 15, no. 1, pp. 686-698, January 2016.

[32] H. Kim, H. Lee, M. Ahn, H. B. Kong, and I. Lee, "Joint Subcarrier and Power Allocation Methods in Full Duplex Wireless Powered Communication Networks for OFDM Systems," IEEE Transactions on Wireless Communications, vol. 15, no. 7, pp. 4745-4753, July 2016.

[33] J. Moon, H. Lee, C. Song, and I. Lee, "Secrecy Performance Optimization for Wireless Powered Communication Networks With an Energy Harvesting Jammer," IEEE Transactions on Communications, vol. 65 , no. 2, pp. 764-774, February 2017.

[34] K. Xiong, C. Chen, G. Qu, P. Fan, and K. Letaief, "Group Cooperation with Optimal Resource Allocation in Wireless Powered Communication Networks," IEEE Transactions on Wireless Communications, vol. PP, no. 99, pp. 1-1, 2017.

[35] B. H. Choi, V. X. Thai, E. S. Lee, J. H. Kim, and C. T. Rim, "DipoleCoil-Based Wide-Range Inductive Power Transfer Systems for Wireless Sensors," IEEE Transactions on Industrial Electronics, vol. 63, no. 5, pp. 3158-3167, May 2016.

[36] V. Jiwariyavej, T. Imura, and Y. Hori, "Coupling Coefficients Estimation of Wireless Power Transfer System via Magnetic Resonance Coupling Using Information From Either Side of the System," IEEE Journal of Emerging and Selected Topics in Power Electronics, vol. 3, no. 1, pp. 191-200, March 2015.

[37] P. S. Riehl, A. Satyamoorthy, H. Akram, Y. C. Yen, J. C. Yang, B. Juan, C. M. Lee, F. C. Lin, V. Muratov, W. Plumb, and P. F. Tustin, "Wireless Power Systems for Mobile Devices Supporting Inductive and Resonant Operating Modes," IEEE Transactions on Microwave Theory and Techniques, vol. 63, no. 3, pp. 780-790, March 2015.

[38] M. Pinuela, D. C. Yates, S. Lucyszyn, and P. D. Mitcheson, "Maximizing DC-to-Load Efficiency for Inductive Power Transfer," IEEE Transactions on Power Electronics, vol. 28, no. 5, pp. 2437-2447, May 2013.

[39] H. Liu, "Maximizing Efficiency of Wireless Power Transfer With Resonant Inductive Coupling," 2011.

[40] C. Zheng, H. Ma, J. S. Lai, and L. Zhang, "Design Considerations to Reduce Gap Variation and Misalignment Effects for the Inductive Power Transfer System," IEEE Transactions on Power Electronics, vol. 30, no. 11, pp. 6108-6119, November 2015.

[41] Z. Yan, Y. Li, C. Zhang, and Q. Yang, "Influence Factors Analysis and Improvement Method on Efficiency of Wireless Power Transfer Via Coupled Magnetic Resonance," IEEE Transactions on Magnetics, vol. 50, no. 4, pp. 1-4, April 2014.

[42] J. M. Miller, O. C. Onar, and M. Chinthavali, "Primary-Side Power Flow Control of Wireless Power Transfer for Electric Vehicle Charging," IEEE Journal of Emerging and Selected Topics in Power Electronics, vol. 3, no. 1, pp. 147-162, March 2015.

[43] A. Kurs, A. Karalis, R. Moffatt, J. D. Joannopoulos, P. Fisher, and M. Soljačić, "Wireless Power Transfer via Strongly Coupled Magnetic Resonances," Science, vol. 317, no. 5834, pp. 83-86, 2007.

[44] H. Hwang, J. Moon, B. Lee, C. h. Jeong, and S. w. Kim, "An analysis of magnetic resonance coupling effects on wireless power transfer by coil inductance and placement," IEEE Transactions on Consumer Electronics, vol. 60, no. 2, pp. 203-209, May 2014.

[45] M. Q. Nguyen, D. Plesa, S. Rao, and J. C. Chiao, "A multi-input and multi-output wireless energy transfer system," in 2014 IEEE MTT-S International Microwave Symposium (IMS2014), June 2014, pp. 1-3.

[46] M. Q. Nguyen, Y. Chou, D. Plesa, S. Rao, and J. C. Chiao, "MultipleInputs and Multiple-Outputs Wireless Power Combining and Delivering Systems," IEEE Transactions on Power Electronics, vol. 30, no. 11, pp. 6254-6263, November 2015.

[47] J. H. Kim, H. Y. Yu, and C. Cha, "Efficiency enhancement using beam forming array antenna for microwave-based wireless energy transfer," in 2014 IEEE Wireless Power Transfer Conference, May 2014, pp. 288-291.

[48] W. C. Brown and R. H. George, "Rectification of microwave power," IEEE Spectrum, vol. 1, no. 10, pp. 92-97, Oct 1964.

[49] W. C. Brown, "The History of Power Transmission by Radio Waves," IEEE Transactions on Microwave Theory and Techniques, vol. 32, no. 9, pp. 1230-1242, Sep 1984.
[50] _ "Status of the Microwave Power Transmission Components for the Solar Power Satellite," IEEE Transactions on Microwave Theory and Techniques, vol. 29, no. 12, pp. 1319-1327, Dec 1981.

[51] W. Brown, "Recent advances in key microwave components that impact the design and deployment of the solar power satellite system," in 1984 Antennas and Propagation Society International Symposium, vol. 22, June 1984, pp. 339-340.

[52] R. M. Dickinson, "Performance of a High-Power, 2.388-GHz Receiving Array in Wireless Power Transmission Over $1.54 \mathrm{~km}$," in 1976 IEEEMTT-S International Microwave Symposium, June 1976, pp. 139-141.

[53] P. S. Yedavalli, T. Riihonen, X. Wang, and J. M. Rabaey, "Far-Field RF Wireless Power Transfer with Blind Adaptive Beamforming for Internet of Things Devices," IEEE Access, vol. 5, pp. 1743-1752, 2017.

[54] T. Mitani, S. Kawashima, and T. Nishimura, "Analysis of Voltage Doubler Behavior of 2.45-GHz Voltage Doubler-Type Rectenna," IEEE Transactions on Microwave Theory and Techniques, vol. 65, no. 4, pp. 1051-1057, April 2017.

[55] N. Moraitis, P. N. Vasileiou, C. G. Kakoyiannis, A. Marousis, A. G. Kanatas, and P. Constantinou, "Radio Planning of Single-Frequency Networks for Broadcasting Digital TV in Mixed-Terrain Regions," IEEE Antennas and Propagation Magazine, vol. 56, no. 6, pp. 123141, December 2014.

[56] M. Morelli and M. Moretti, "A Maximum Likelihood Approach for SSS Detection in LTE Systems," IEEE Transactions on Wireless Communications, vol. 16, no. 4, pp. 2423-2433, April 2017.

[57] L. Sanabria-Russo, J. Barcelo, B. Bellalta, and F. Gringoli, "A High Efficiency MAC Protocol for WLANs: Providing Fairness in Dense Scenarios," IEEE/ACM Transactions on Networking, vol. 25, no. 1, pp. 492-505, February 2017.

[58] R. Ford, M. Zhang, M. Mezzavilla, S. Dutta, S. Rangan, and M. Zorzi, "Achieving Ultra-Low Latency in 5G Millimeter Wave Cellular Networks," IEEE Communications Magazine, vol. 55, no. 3, pp. 196-203, March 2017.

[59] M. Pasca, S. D’Amico, V. Chironi, L. Catarinucci, D. de Donno, R. Colella, and L. Tarricone, "A $-19 \mathrm{dbm}$ sensitivity integrated rf$\mathrm{dc}$ converter with regulated output voltage for powering uhf wireless sensors," in 2015 6th International Workshop on Advances in Sensors and Interfaces (IWASI), June 2015, pp. 168-171.

[60] S. Chatterjee and M. Tarique, "A 100-nw sensitive rf-to-dc cmos rectifier for energy harvesting applications," in 2016 29th International Conference on VLSI Design and 2016 15th International Conference on Embedded Systems (VLSID), January 2016, pp. 557-558.

[61] N. M. Pletcher, S. Gambini, and J. Rabaey, "A $52 m u$ w wake-up receiver with $-72 \mathrm{dbm}$ sensitivity using an uncertain-if architecture," IEEE Journal of Solid-State Circuits, vol. 44, no. 1, pp. 269-280, January 2009.

[62] R. Ni, K. Mayaram, and T. S. Fiez, “A $915 \mathrm{mhz}, 6 \mathrm{mb} / \mathrm{s}, 80 \mathrm{pj} / \mathrm{b}$ bfsk receiver with $-76 \mathrm{dbm}$ sensitivity for high data rate wireless sensor networks," in 2014 Symposium on VLSI Circuits Digest of Technical Papers, June 2014, pp. 1-2.

[63] T. Zhang, L. An, Y. Chen, and K. K. Chai, "Aggregate interference statistical modeling and user outage analysis of heterogeneous cellular networks," in 2014 IEEE International Conference on Communications (ICC), June 2014, pp. 1260-1265.

[64] J. Hu, L. L. Yang, and L. Hanzo, "Distributed Multistage CooperativeSocial-Multicast-Aided Content Dissemination in Random Mobile Networks," IEEE Transactions on Vehicular Technology, vol. 64, no. 7, pp. 3075-3089, July 2015.

[65] J. Hu, L. L. Yang, H. V. Poor, and L. Hanzo, "Bridging the Social and Wireless Networking Divide: Information Dissemination in Integrated Cellular and Opportunistic Networks," IEEE Access, vol. 3, pp. 1809$1848,2015$.

[66] J. Hu, L. L. Yang, and L. Hanzo, "Delay Analysis of Social Group Multicast-Aided Content Dissemination in Cellular System," IEEE Transactions on Communications, vol. 64, no. 4, pp. 1660-1673, April 2016.

[67] — , "Energy-Efficient Cross-Layer Design of Wireless Mesh Networks for Content Sharing in Online Social Networks," IEEE Transactions on Vehicular Technology, vol. PP, no. 99, pp. 1-1, 2017.

[68] K. Yang, Q. Yu, S. Leng, B. Fan, and F. Wu, "Data and Energy Integrated Communication Networks for Wireless Big Data," IEEE Access, vol. 4, pp. 713-723, 2016.

[69] H. Lee, J. Kwon, W. Lim, W. Lee, H. Kang, K. C. Hwang, K. Y. Lee, C. s. Park, and Y. Yang, "Optimized Current of the Peaking Amplifier for Two-Stage Doherty Power Amplifier," IEEE Transactions on Microwave Theory and Techniques, vol. 65, no. 1, pp. 209-217, Jan 2017. 
[70] M. V. Berry and N. L. Balazs, "Nonspreading wave packets," American Journal of Physics, vol. 47, no. 3, pp. 264-267, March 1979.

[71] G. A. Siviloglou, J. Broky, A. Dogariu, and D. N. Christodoulides, "Observation of Accelerating Airy Beams," Phys. Rev. Lett., vol. 99, p. 213901, November 2007.

[72] I. D. Chremmos, G. Fikioris, and N. K. Efremidis, "Accelerating and Abruptly-Autofocusing Beam Waves in the Fresnel Zone of Antenna Arrays," IEEE Transactions on Antennas and Propagation, vol. 61, no. 10, pp. 5048-5056, Oct 2013.

[73] E. L. Firrao, A. J. Annema, F. E. van Vliet, and B. Nauta, "Hardware Implementation Overhead of Switchable Matching Networks," IEEE Transactions on Circuits and Systems I: Regular Papers, vol. 64, no. 5, pp. 1152-1163, May 2017.

[74] J. Charthad, N. Dolatsha, A. Rekhi, and A. Arbabian, "System-Level Analysis of Far-Field Radio Frequency Power Delivery for mm-Sized Sensor Nodes," IEEE Transactions on Circuits and Systems I: Regular Papers, vol. 63, no. 2, pp. 300-311, February 2016.

[75] Z. Hameed and K. Moez, "Hybrid Forward and Backward ThresholdCompensated RF-DC Power Converter for RF Energy Harvesting," IEEE Journal on Emerging and Selected Topics in Circuits and Systems, vol. 4, no. 3, pp. 335-343, September 2014.

[76] M. Gastpar, "On Capacity Under Receive and Spatial Spectrum-Sharing Constraints," IEEE Transactions on Information Theory, vol. 53, no. 2, pp. 471-487, February 2007.

[77] A. Tandon, M. Motani, and L. R. Varshney, "On code design for simultaneous energy and information transfer," in 2014 Information Theory and Applications Workshop (ITA). IEEE, feb 2014, pp. 1-6.

[78] J. Y. Wang, Z. Yang, Y. Wang, and M. Chen, "On the Performance of Spatial Modulation-Based Optical Wireless Communications," IEEE Photonics Technology Letters, vol. 28, no. 19, pp. 2094-2097, October 2016.

[79] L. Hanzo, M. El-Hajjar, and O. Alamri, "Near-Capacity Wireless Transceivers and Cooperative Communications in the MIMO Era: Evolution of Standards, Waveform Design, and Future Perspectives," Proceedings of the IEEE, vol. 99, no. 8, pp. 1343-1385, August 2011.

[80] Y. Dong, M. J. Hossain, and J. Cheng, "Joint Power Control and Time Switching for SWIPT Systems With Heterogeneous QoS Requirements," IEEE Communications Letters, vol. 20, no. 2, pp. 328 331, February 2016.

[81] F. Zhou, Z. Li, J. Cheng, Q. Li, and J. Si, "Robust AN-Aided Beamforming and Power Splitting Design for Secure MISO Cognitive Radio With SWIPT," IEEE Transactions on Wireless Communications, vol. 16, no. 4, pp. 2450-2464, April 2017.

[82] F. Sohrabi and W. Yu, "Hybrid Analog and Digital Beamforming for mmWave OFDM Large-Scale Antenna Arrays," IEEE Journal on Selected Areas in Communications, vol. PP, no. 99, pp. 1-1, 2017.

[83] B. Clerckx, "Wireless information and power transfer: Nonlinearity, waveform design, and rate-energy tradeoff," IEEE Transactions on Signal Processing, vol. 66, no. 4, pp. 847-862, February 2018.

[84] K. Huang, C. Zhong, and G. Zhu, "Some new research trends in wirelessly powered communications," IEEE Wireless Communications, vol. 23, no. 2, pp. 19-27, April 2016

[85] J. Xu and R. Zhang, "Energy Beamforming With One-Bit Feedback," IEEE Transactions on Signal Processing, vol. 62, no. 20, pp. 53705381, October 2014

[86] J. Hu, Y. Xue, Q. Yu, and K. Yang, "A Joint Time Allocation and UE Scheduling Algorithm for Full-Duplex Wireless Powered Communication Networks," in 2017 IEEE 86th Vehicular Technology Conference (VTC-Fall), September 2017, pp. 1-5

[87] M. Maso, C. F. Liu, C. H. Lee, T. Q. S. Quek, and L. S. Cardoso, "Energy-Recycling Full-Duplex Radios for Next-Generation Networks," IEEE Journal on Selected Areas in Communications, vol. 33 , no. 12, pp. 2948-2962, December 2015.

[88] D. P. Venmani, O. L. Moult, F. Deletre, Y. Lagadec, and Y. Morlon, "On the role of network synchronization for future cellular networks: an operator's perspective," IEEE Communications Magazine, vol. 54, no. 9, pp. 58-64, September 2016.

[89] L. R. Varshney, "On energy/information cross-layer architectures," in 2012 IEEE International Symposium on Information Theory Proceedings, July 2012, pp. 1356-1360.

[90] D. Alanis, J. Hu, P. Botsinis, Z. Babar, S. X. Ng, and L. Hanzo, "Quantum-Assisted Joint Multi-Objective Routing and Load Balancing for Socially-Aware Networks," IEEE Access, vol. 4, pp. 9993-10028, November 2016.

[91] J. Wu, I. Bisio, C. Gniady, E. Hossain, M. Valla, and H. Li, "ContextAware Networking and Communications: Part 1 [Guest Editorial]," IEEE Communications Magazine, vol. 52, no. 6, pp. 14-15, June 2014.
[92] K. Niotaki, A. Collado, A. Georgiadis, S. Kim, and M. M. Tentzeris, "Solar/Electromagnetic Energy Harvesting and Wireless Power Transmission," Proceedings of the IEEE, vol. 102, no. 11, pp. 1712-1722, November 2014.

[93] X. Lu, P. Wang, D. Niyato, D. I. Kim, and Z. Han, "Wireless Charging Technologies: Fundamentals, Standards, and Network Applications," IEEE Communications Surveys Tutorials, vol. 18, no. 2, pp. 14131452, Secondquarter 2016.

[94] S. Ulukus, A. Yener, E. Erkip, O. Simeone, M. Zorzi, P. Grover and K. Huang, "Energy Harvesting Wireless Communications: A Review of Recent Advances," IEEE Journal on Selected Areas in Communications, vol. 33, no. 3, pp. 360-381, March 2015.

[95] M. L. Ku, W. Li, Y. Chen, and K. J. R. Liu, "Advances in Energy Harvesting Communications: Past, Present, and Future Challenges," IEEE Communications Surveys Tutorials, vol. 18, no. 2, pp. 13841412, Secondquarter 2016.

[96] X. Lu, P. Wang, D. Niyato, D. I. Kim, and Z. Han, "Wireless Networks With RF Energy Harvesting: A Contemporary Survey," IEEE Communications Surveys Tutorials, vol. 17, no. 2, pp. 757-789, Secondquarter 2015.

[97] M. D. Renzo and W. Lu, "System-Level Analysis and Optimization of Cellular Networks With Simultaneous Wireless Information and Power Transfer: Stochastic Geometry Modeling," IEEE Transactions on Vehicular Technology, vol. 66, no. 3, pp. 2251-2275, March 2017.

[98] D. H. Chen and Y. C. He, "Full-Duplex Secure Communications in Cellular Networks With Downlink Wireless Power Transfer," IEEE Transactions on Communications, vol. 66, no. 1, pp. 265-277, January 2018.

[99] J. An, K. Yang, J. Wu, N. Ye, S. Guo, and Z. Liao, "Achieving sustainable ultra-dense heterogeneous networks for 5g," IEEE Communications Magazine, vol. 55, no. 12, pp. 84-90, December 2017.

[100] X. Liu, Z. Li, and C. Wang, "Secure Decode-and-Forward Relay SWIPT Systems With Power Splitting Scheme," IEEE Transactions on Vehicular Technology, pp. 1-1, 2018.

[101] Y. Zhao, D. Wang, J. Hu, and K. Yang, "H-AP Deployment for Joint Wireless Information and Energy Transfer in Smart Cities," IEEE Transactions on Vehicular Technology, pp. 1-1, 2018.

[102] K. Lv, J. Hu, Q. Yu, and K. Yang, "Throughput Maximization and Fairness Assurance in Data and Energy Integrated Communication Networks," IEEE Internet of Things Journal, vol. 5, no. 2, pp. 636-644, April 2018.

[103] Y. Ye, Y. Li, D. Wang, F. Zhou, R. Q. Hu, and H. Zhang, "Optimal Transmission Schemes for DF Relaying Networks Using SWIPT," IEEE Transactions on Vehicular Technology, pp. 1-1, 2018.

[104] Y. Zeng, B. Clerckx, and R. Zhang, "Communications and Signals Design for Wireless Power Transmission," IEEE Transactions on Communications, vol. 65, no. 5, pp. 2264-2290, May 2017.

[105] Q. Li, Q. Zhang, and J. Qin, "Robust Tomlinson-Harashima Precoding With Gaussian Uncertainties for SWIPT in MIMO Broadcast Channels," IEEE Transactions on Signal Processing, vol. 65, no. 6, pp. 1399-1411, March 2017.

[106] C. Cano, A. Pittolo, D. Malone, L. Lampe, A. M. Tonello, and A. G. Dabak, "State of the Art in Power Line Communications: From the Applications to the Medium," IEEE Journal on Selected Areas in Communications, vol. 34, no. 7, pp. 1935-1952, July 2016.

[107] J. Wu, H. Wu, C. Li, W. Li, X. He, and C. Xia, "Advanced FourPair Architecture With Input Current Balance Function for Power Over Ethernet (PoE) System," IEEE Transactions on Power Electronics, vol. 28, no. 5, pp. 2343-2355, May 2013.

[108] L. R. Varshney, "Transporting information and energy simultaneously," in 2008 IEEE International Symposium on Information Theory, July 2008, pp. 1612-1616.

[109] C. Pimentel and F. Alajaji, "Packet-Based Modeling of Reed-Solomon Block-Coded Correlated Fading Channels Via a Markov Finite Queue Model," IEEE Transactions on Vehicular Technology, vol. 58, no. 7, pp. 3124-3136, September 2009.

[110] M. N. Abadi and L. Cardeno, "Renyi Entropies and Large Deviations for the First Match Function," IEEE Transactions on Information Theory, vol. 61, no. 4, pp. 1629-1639, April 2015.

[111] A. I. Barbero, E. Rosnes, G. Yang, and O. Ytrehus, "Constrained codes for passive RFID communication," in 2011 Information Theory and Applications Workshop. IEEE, feb 2011, pp. 1-9.

[112] A. M. Fouladgar, O. Simeone, and E. Erkip, "Constrained Codes for Joint Energy and Information Transfer," IEEE Transactions on Communications, vol. 62, no. 6, pp. 2121-2131, jun 2014.

[113] A. Tandon, M. Motani, and L. R. Varshney, "Constant subblock composition codes for simultaneous energy and information transfer," 
in 2014 Eleventh Annual IEEE International Conference on Sensing, Communication, and Networking Workshops (SECON Workshops), June 2014, pp. 45-50.

[114] — "Real-time simultaneous energy and information transfer," in 2015 IEEE International Symposium on Information Theory (ISIT), June 2015, pp. 1124-1128.

[115] _ _ "Subblock-Constrained Codes for Real-Time Simultaneous Energy and Information Transfer," IEEE Transactions on Information Theory, vol. 62, no. 7, pp. 4212-4227, July 2016.

[116] V. Vorapipat, C. S. Levy, and P. M. Asbeck, "Voltage Mode Doherty Power Amplifier," IEEE Journal of Solid-State Circuits, vol. PP, no. 99, pp. 1-10, 2017.

[117] S. Hu, S. Kousai, J. S. Park, O. L. Chlieh, and H. Wang, "Design of A Transformer-Based Reconfigurable Digital Polar Doherty Power Amplifier Fully Integrated in Bulk CMOS," IEEE Journal of SolidState Circuits, vol. 50, no. 5, pp. 1094-1106, May 2015.

[118] S. M. Yoo, J. S. Walling, O. Degani, B. Jann, R. Sadhwani, J. C. Rudell, and D. J. Allstot, "A Class-G Switched-Capacitor RF Power Amplifier," IEEE Journal of Solid-State Circuits, vol. 48, no. 5, pp. 1212-1224, May 2013.

[119] E. Kaymaksut and P. Reynaert, "Dual-Mode CMOS Doherty LTE Power Amplifier With Symmetric Hybrid Transformer," IEEE Journal of Solid-State Circuits, vol. 50, no. 9, pp. 1974-1987, Sept 2015.

[120] L. Ye, J. Chen, L. Kong, E. Alon, and A. M. Niknejad, "Design Considerations for a Direct Digitally Modulated WLAN Transmitter With Integrated Phase Path and Dynamic Impedance Modulation," IEEE Journal of Solid-State Circuits, vol. 48, no. 12, pp. 3160-3177, Dec 2013.

[121] W. Yuan, V. Aparin, J. Dunworth, L. Seward, and J. S. Walling, "A Quadrature Switched Capacitor Power Amplifier," IEEE Journal of Solid-State Circuits, vol. 51, no. 5, pp. 1200-1209, May 2016.

[122] M. P. Gopalrao and A. A. Yadav, "CMOS RF Power Amplifier design for wideband frequency," in 2016 International Conference on Automatic Control and Dynamic Optimization Techniques (ICACDOT), Sept 2016, pp. 930-934.

[123] H. Madureira, N. Deltimple, S. Haddad, D. Belot, and E. Kerhervé, "Design of a wideband $1.5 \mathrm{GHz}$ to $3 \mathrm{GHz}$ class EF2 power amplifier," in 2014 IEEE 12th International New Circuits and Systems Conference (NEWCAS), June 2014, pp. 173-176.

[124] A. Mazzanti, L. Larcher, R. Brama, and F. Svelto, "Analysis of reliability and power efficiency in cascode class-E PAs," IEEE Journal of Solid-State Circuits, vol. 41, no. 5, pp. 1222-1229, May 2006.

[125] J. Tan, C. H. Heng, and Y. Lian, "Design of Efficient Class-E Power Amplifiers for Short-Distance Communications," IEEE Transactions on Circuits and Systems I: Regular Papers, vol. 59, no. 10, pp. 2210-2220, Oct 2012.

[126] K. Takenaka, T. Sato, H. Matsumoto, M. Kawashima, and N. Nakajima, "New compact Doherty power amplifier design for handset applications," in 2017 IEEE Topical Conference on RF/Microwave Power Amplifiers for Radio and Wireless Applications (PAWR), Jan 2017, pp. 81-83.

[127] D. Kang, D. Kim, Y. Cho, J. Kim, B. Park, C. Zhao, and B. Kim, "1.6-2.1 GHz broadband Doherty power amplifiers for LTE handset applications," in 2011 IEEE MTT-S International Microwave Symposium, June 2011, pp. 1-4.

[128] Y. Cho, K. Moon, J. Kim, B. Park, and B. Kim, "Linear Doherty power amplifier with adaptive bias circuit for average power-tracking," in 2016 IEEE MTT-S International Microwave Symposium (IMS), May 2016, pp. 1-3.

[129] R. J. Trew, "SiC and GaN Transistors - Is There One Winner for Microwave Power Applications?" Proceedings of the IEEE, vol. 90 , no. 6, pp. 1032-1047, Jun 2002.

[130] C. X. Mao, S. Gao, Y. Wang, and B. S. Izquierdo, "A Novel Multi-Band Directional Antenna for Wireless Communications," IEEE Antennas and Wireless Propagation Letters, vol. PP, no. 99, pp. 1-1, 2016.

[131] G. C. Huang, M. F. Iskander, and M. Hoque, "Advanced antenna array designs for directional networks," in MILCOM 2016 - 2016 IEEE Military Communications Conference, Nov 2016, pp. 204-207.

[132] W. Wang, M. Yan, J. Wang, H. Yuan, Y. Pang, C. Xu, S. Qu, M. Feng, H. Ma, J. Wang, H. Chen, and Z. Li, "Linear-to-linear high directional antenna using transmission polarization metasurface," in 2016 Progress in Electromagnetic Research Symposium (PIERS), Aug 2016, pp. 28152818

[133] L. Jiang, Y. Chun, C. Qi, and L. Bo, "Millimetre wave circularly polarized holographic antenna based on artificial impedance surface," in IET International Radar Conference 2015, Oct 2015, pp. 1-3.
[134] T. Su, Q. Zhang, R. Chen, and C. Sun, "Novel Design of Surface-Wave Holographic Antenna Miniaturization," IEEE Antennas and Wireless Propagation Letters, vol. 14, pp. 1077-1080, 2015.

[135] J. Schafer, M. Dittrich, H. Gulan, and T. Zwick, "Planar frequency scanning holographic antenna for FMCW-radar applications at 240 GHz," in 2016 IEEE International Symposium on Antennas and Propagation (APSURSI), June 2016, pp. 1401-1402.

[136] X. Chu, Q. Sun, J. Wang, P. Lu, and W. X. X. Xu, "Generating a BesselGaussian beam for the application in optical engineering," Scientific Reports, vol. 5, no. 6, p. 18665, December 2015.

[137] O. M. Ramahi, T. S. Almoneef, M. AlShareef, and M. S. Boybay, "Metamaterial particles for electromagnetic energy harvesting," Applied Physics Letters, vol. 101, no. 17, p. 173903, 2012. [Online] Available: http://dx.doi.org/10.1063/1.4764054

[138] A. M. Hawkes, A. R. Katko, and S. A. Cummer, "A microwave metamaterial with integrated power harvesting functionality," Applied Physics Letters, vol. 103, no. 16, p. 163901, 2013. [Online]. Available: http://dx.doi.org/10.1063/1.4824473

[139] T. S. Almoneef and O. M. Ramahi, "Metamaterial electromagnetic energy harvester with near unity efficiency," Applied Physics Letters, vol. 106, no. 15, p. 153902, 2015. [Online]. Available: http://dx.doi.org/10.1063/1.4916232

[140] B. Alavikia, T. S. Almoneef, and O. M. Ramahi, "Complementary split ring resonator arrays for electromagnetic energy harvesting," Applied Physics Letters, vol. 107, no. 3, p. 033902, 2015. [Online]. Available: http://dx.doi.org/10.1063/1.4927238

[141] G. T. O. Tekam, V. Ginis, J. Danckaert, and P. Tassin, "Designing an efficient rectifying cut-wire metasurface for electromagnetic energy harvesting," Applied Physics Letters, vol. 110, no. 8, p. 083901, 2017. [Online]. Available: http://dx.doi.org/10.1063/1.4976804

[142] G. Palumbo, "The Design Of CMOS Radio-Frequency Integrated Circuits," Communications Engineer, vol. 2, no. 4, pp. 47-47, 2004.

[143] Y. Huang, N. Shinohara, and T. Mitani, "Impedance Matching in Wireless Power Transfer," IEEE Transactions on Microwave Theory and Techniques, vol. 65, no. 2, pp. 582-590, February 2017.

[144] M. K. Y. N and P. Duraiswamy, "On the optimization of GaN Schottky Diode rectifier circuit topologies for high efficiency Wireless Power Transmission," in 2016 Asia-Pacific Microwave Conference (APMC), Dec 2016, pp. 1-4.

[145] T. Le, K. Mayaram, and T. Fiez, "Efficient Far-Field Radio Frequency Energy Harvesting for Passively Powered Sensor Networks," IEEE Journal of Solid-State Circuits, vol. 43, no. 5, pp. 1287-1302, May 2008.

[146] L. G. de Carli, Y. Juppa, A. J. Cardoso, C. Galup-Montoro, and M. C. Schneider, "Maximizing the Power Conversion Efficiency of UltraLow-Voltage CMOS Multi-Stage Rectifiers," IEEE Transactions on Circuits and Systems I: Regular Papers, vol. 62, no. 4, pp. 967-975, April 2015.

[147] J. Yi, W. H. Ki, and C. Y. Tsui, "Analysis and Design Strategy of UHF Micro-Power CMOS Rectifiers for Micro-Sensor and RFID Applications," IEEE Transactions on Circuits and Systems I: Regular Papers, vol. 54, no. 1, pp. 153-166, Jan 2007.

[148] C. Song, Y. Huang, P. Carter, J. Zhou, S. Yuan, Q. Xu, and M. Kod, "A Novel Six-Band Dual CP Rectenna Using Improved Impedance Matching Technique for Ambient RF Energy Harvesting," IEEE Transactions on Antennas and Propagation, vol. 64, no. 7, pp. 3160-3171, July 2016.

[149] S. Yoshida, A. Miyachi, R. Kishikawa, M. Horibe, K. Nishikawa, and S. Kawasaki, "C band GaN diode rectifier with 3W DC output for high power microwave power transmission applications," in 2016 IEEE MTT-S International Microwave Symposium (IMS), May 2016, pp. 1-4.

[150] Y. Y. Hu, X. Yu, and S. Sun, "Design of a closely-spaced-element rectenna for low-power wireless energy harvesting," in 2016 IEEE International Workshop on Electromagnetics: Applications and Student Innovation Competition (iWEM), May 2016, pp. 1-2.

[151] A. M. AbdelTawab and A. Khattab, "Efficient multi-band energy Harvesting circuit for Wireless Sensor nodes," in 2016 Fourth International Japan-Egypt Conference on Electronics, Communications and Computers (JEC-ECC), May 2016, pp. 75-78.

[152] T. Moura, W. Jang, P. Cruz, D. Belo, N. B. Carvalho, S. Chevtchenko, B. Janke, and J. Wurfl, "MMIC GaN power amplifier with bidirectional operation for RF-to-DC conversion," in 2016 IEEE MTT-S International Wireless Symposium (IWS), March 2016, pp. 1-4.

[153] S. Dehghani, S. Abbasian, and T. Johnson, "Tracking load to optimize power efficiency in RF to DC rectifier circuits," in 2015 IEEE Wireless Power Transfer Conference (WPTC), May 2015, pp. 1-3.

[154] D. Y. Hyun, C. S. Lim, R. Y. Kim, and D. S. Hyun, "Modeling and Control of Two-Stage Inverter for Battery Energy Storage System," in 
2013 IEEE Vehicle Power and Propulsion Conference (VPPC), Oct 2013, pp. 1-5.

[155] B. D. Valle, C. T. Wentz, and R. Sarpeshkar, "An Area and PowerEfficient Analog Li-Ion Battery Charger Circuit," IEEE Transactions on Biomedical Circuits and Systems, vol. 5, no. 2, pp. 131-137, April 2011.

[156] R. Pagano, M. Baker, and R. E. Radke, "A 0.18- $\mu m$ Monolithic Li-Ion Battery Charger for Wireless Devices Based on Partial Current Sensing and Adaptive Reference Voltage," IEEE Journal of Solid-State Circuits, vol. 47, no. 6, pp. 1355-1368, June 2012.

[157] Y. S. Hwang, S. C. Wang, F. C. Yang, and J. J. Chen, "New Compact CMOS Li-Ion Battery Charger Using Charge-Pump Technique for Portable Applications," IEEE Transactions on Circuits and Systems I: Regular Papers, vol. 54, no. 4, pp. 705-712, April 2007.

[158] Y. Qiu, C. V. Liempd, B. O. het Veld, P. G. Blanken, and C. V. Hoof, "5uW-to-10 $\mathrm{mW}$ input power range inductive boost converter for indoor photovoltaic energy harvesting with integrated maximum power point tracking algorithm," in 2011 IEEE International Solid-State Circuits Conference, Feb 2011, pp. 118-120.

[159] R. Enne, M. Nikolic, and H. Zimmermann, "A maximum power-point tracker without digital signal processing in $0.35+\mathrm{x} 03 \mathrm{BC} ; \mathrm{m}$ CMOS for automotive applications," in 2012 IEEE International Solid-State Circuits Conference, Feb 2012, pp. 102-104.

[160] T. H. Tsai and K. Chen, "A $3.4 \mathrm{~mW}$ photovoltaic energy-harvesting charger with integrated maximum power point tracking and battery management," in 2013 IEEE International Solid-State Circuits Conference Digest of Technical Papers, Feb 2013, pp. 72-73.

[161] P. Grover and A. Sahai, "Shannon meets Tesla: Wireless information and power transfer," in 2010 IEEE International Symposium on Information Theory, June 2010, pp. 2363-2367.

[162] X. Zhou, R. Zhang, and C. K. Ho, "Wireless Information and Power Transfer: Architecture Design and Rate-Energy Tradeoff," IEEE Transactions on Communications, vol. 61, no. 11, pp. 4754-4767, November 2013.

[163] L. Liu, R. Zhang, and K. C. Chua, "Wireless Information and Power Transfer: A Dynamic Power Splitting Approach," IEEE Transactions on Communications, vol. 61, no. 9, pp. 3990-4001, September 2013.

[164] R. Zhang and C. K. Ho, "MIMO Broadcasting for Simultaneous Wireless Information and Power Transfer," IEEE Transactions on Wireless Communications, vol. 12, no. 5, pp. 1989-2001, May 2013.

[165] Z. Xiang and M. Tao, "Robust Beamforming for Wireless Information and Power Transmission," IEEE Wireless Communications Letters, vol. 1, no. 4, pp. 372-375, August 2012.

[166] B. Clerckx and E. Bayguzina, "Waveform Design for Wireless Power Transfer," IEEE Transactions on Signal Processing, vol. 64, no. 23, pp. 6313-6328, Dec 2016.

[167] A. Collado and A. Georgiadis, "Optimal Waveforms for Efficient Wireless Power Transmission," IEEE Microwave and Wireless Components Letters, vol. 24, no. 5, pp. 354-356, May 2014.

[168] B. Clerckx, "Waveform Optimization for SWIPT with Nonlinear Energy Harvester Modeling," in WSA 2016; 20th International ITG Workshop on Smart Antennas, March 2016, pp. 1-5.

[169] D. I. Kim, J. H. Moon, and J. J. Park, "New SWIPT Using PAPR How It Works," IEEE Wireless Communications Letters, vol. 5, no. 6, pp. 672-675, Dec 2016.

[170] T. Paing, J. Shin, R. Zane, and Z. Popovic, "Resistor Emulation Approach to Low-Power RF Energy Harvesting," IEEE Transactions on Power Electronics, vol. 23, no. 3, pp. 1494-1501, May 2008.

[171] E. Boshkovska, D. W. K. Ng, N. Zlatanov, and R. Schober, "Practical Non-Linear Energy Harvesting Model and Resource Allocation for SWIPT Systems," IEEE Communications Letters, vol. 19, no. 12, pp. 2082-2085, Dec 2015.

[172] E. Boshkovska, R. Morsi, D. W. K. Ng, and R. Schober, "Power allocation and scheduling for SWIPT systems with non-linear energy harvesting model," in 2016 IEEE International Conference on Communications (ICC), May 2016, pp. 1-6.

[173] H. Ju and R. Zhang, "Throughput Maximization in Wireless Powered Communication Networks," IEEE Transactions on Wireless Communications, vol. 13, no. 1, pp. 418-428, January 2014.

[174] L. Liu, R. Zhang, and K. C. Chua, "Multi-Antenna Wireless Powered Communication With Energy Beamforming," IEEE Transactions on Communications, vol. 62, no. 12, pp. 4349-4361, Dec 2014.

[175] H. Ju and R. Zhang, "Optimal Resource Allocation in Full-Duplex Wireless-Powered Communication Network," IEEE Transactions on Communications, vol. 62, no. 10, pp. 3528-3540, Oct 2014

[176] Y. L. Che, L. Duan, and R. Zhang, "Spatial Throughput Maximization of Wireless Powered Communication Networks," IEEE Journal on
Selected Areas in Communications, vol. 33, no. 8, pp. 1534-1548, Aug 2015.

[177] J. Kim, H. Lee, C. Song, T. Oh, and I. Lee, "Sum Throughput Maximization for Multi-User MIMO Cognitive Wireless Powered Communication Networks," IEEE Transactions on Wireless Communications, vol. PP, no. 99, pp. 1-1, 2016.

[178] B. Koo and D. Park, "Interference Alignment and Wireless Energy Transfer via Antenna Selection," IEEE Communications Letters, vol. 18, no. 4, pp. 548-551, April 2014.

[179] S. Zhao, Q. Li, Q. Zhang, and J. Qin, "Antenna Selection for Simultaneous Wireless Information and Power Transfer in MIMO Systems," IEEE Communications Letters, vol. 18, no. 5, pp. 789-792, May 2014.

[180] K. Liang, L. Zhao, Z. Ding, and H. H. Chen, "Double Side Signal Splitting SWIPT for Downlink CoMP Transmissions With Capacity Limited Backhaul," IEEE Communications Letters, vol. 20, no. 12, pp. 2438-2441, Dec 2016

[181] S. Li, W. Xu, Z. Liu, and J. Lin, "Independent Power Splitting for Interference-Corrupted SIMO SWIPT Systems," IEEE Communications Letters, vol. 20, no. 3, pp. 478-481, March 2016.

[182] G. Zhu, C. Zhong, H. A. Suraweera, G. K. Karagiannidis, Z. Zhang, and T. A. Tsiftsis, "Wireless Information and Power Transfer in Relay Systems With Multiple Antennas and Interference," IEEE Transactions on Communications, vol. 63, no. 4, pp. 1400-1418, April 2015.

[183] H. Liu, K. J. Kim, K. S. Kwak, and H. V. Poor, "Power SplittingBased SWIPT With Decode-and-Forward Full-Duplex Relaying," IEEE Transactions on Wireless Communications, vol. 15, no. 11, pp. 75617577, Nov 2016.

[184] Z. Chu, Z. Zhu, and J. Hussein, "Robust Optimization for AN-Aided Transmission and Power Splitting for Secure MISO SWIPT System," IEEE Communications Letters, vol. 20, no. 8, pp. 1571-1574, Aug 2016.

[185] S. Y. Lee and C. C. Lai, "A 1-V Wideband Low-Power CMOS Active Differential Power Splitter for Wireless Communication," IEEE Transactions on Microwave Theory and Techniques, vol. 55, no. 8, pp. 1593-1600, August 2007.

[186] I.-C. Chang, J.-C. Kao, J.-J. Kuo, and K.-Y. Lin, “An active CMOS one-to-four power splitter for $60-\mathrm{GHz}$ phased-array transmitter," in Microwave Symposium Digest (MTT), 2012 IEEE MTT-S International, June 2012, pp. 1-3.

[187] A. L. Franc, E. Pistono, N. Corrao, D. Gloria, and P. Ferrari, "Compact high-Q, low-loss mmW transmission lines and power splitters in RF CMOS technology," in Microwave Symposium Digest (MTT), 2011 IEEE MTT-S International, June 2011, pp. 1-4.

[188] D. Belot, "Millimeter-wave design in silicon technologies," in 2010 Topical Meeting on Silicon Monolithic Integrated Circuits in RF Systems (SiRF), Jan 2010, pp. 232-238.

[189] J. J. Lee and C. S. Park, "A Slow-Wave Microstrip Line With a HighQ and a High Dielectric Constant for Millimeter-Wave CMOS Application," IEEE Microwave and Wireless Components Letters, vol. 20, no. 7, pp. 381-383, July 2010.

[190] Y. Zhu, K. K. Wong, Y. Zhang, and C. Masouros, "Geometric Power Control for Time-Switching Energy-Harvesting Two-User Interference Channel," IEEE Transactions on Vehicular Technology, vol. 65, no. 12, pp. 9759-9772, Dec 2016

[191] Y. Dong, M. J. Hossain, and J. Cheng, "Joint Power Control and Time Switching for SWIPT Systems With Heterogeneous QoS Requirements," IEEE Communications Letters, vol. 20, no. 2, pp. 328331, Feb 2016

[192] S. Atapattu, H. Jiang, J. Evans, and C. Tellambura, "Time-switching energy harvesting in relay networks," in 2015 IEEE International Conference on Communications (ICC), June 2015, pp. 5416-5421.

[193] Y. Shen, X. Huang, K. S. Kwak, B. Yang, and S. Wang, "SubcarrierPairing-Based Resource Optimization for OFDM Wireless Powered Relay Transmissions With Time Switching Scheme," IEEE Transactions on Signal Processing, vol. 65, no. 5, pp. 1130-1145, March 2017.

[194] S. Timotheou, I. Krikidis, G. Zheng, and B. Ottersten, "Beamforming for MISO Interference Channels with QoS and RF Energy Transfer," IEEE Transactions on Wireless Communications, vol. 13, no. 5, pp. 2646-2658, May 2014.

[195] L. Liu, R. Zhang, and K. C. Chua, "Secrecy Wireless Information and Power Transfer With MISO Beamforming," IEEE Transactions on Signal Processing, vol. 62, no. 7, pp. 1850-1863, April 2014.

[196] Q. Shi, L. Liu, W. Xu, and R. Zhang, "Joint Transmit Beamforming and Receive Power Splitting for MISO SWIPT Systems," IEEE Transactions on Wireless Communications, vol. 13, no. 6, pp. 3269-3280, June 2014 
[197] Z. Wen, X. Liu, N. C. Beaulieu, R. Wang, and S. Wang, "Joint Source and Relay Beamforming Design for Full-Duplex MIMO AF Relay SWIPT Systems," IEEE Communications Letters, vol. 20, no. 2, pp. 320-323, Feb 2016.

[198] A. C. Cirik, J. Xue, S. Biswas, T. Ratnarajah, and M. Sellathurai, "Transceiver design of optimum wirelessly powered full-duplex MIMO interference channel," in 2016 IEEE 17th International Workshop on Signal Processing Advances in Wireless Communications (SPAWC), July 2016, pp. 1-6.

[199] M. M. Zhao, Y. Cai, Q. Shi, M. Hong, and B. Champagne, "Joint Transceiver Designs for Full-Duplex K-Pair MIMO Interference Channel with SWIPT," IEEE Transactions on Communications, vol. PP, no. 99, pp. 1-1, 2016.

[200] J. Park and B. Clerckx, "Joint Wireless Information and Energy Transfer in a Two-User MIMO Interference Channel," IEEE Transactions on Wireless Communications, vol. 12, no. 8, pp. 4210-4221, August 2013.

[201] C. Shen, W. C. Li, and T. H. Chang, "Wireless Information and Energy Transfer in Multi-Antenna Interference Channel," IEEE Transactions on Signal Processing, vol. 62, no. 23, pp. 6249-6264, Dec 2014.

[202] B. Koo and D. Park, "Interference Alignment and Wireless Energy Transfer via Antenna Selection," IEEE Communications Letters, vol. 18, no. 4, pp. 548-551, April 2014.

[203] Z. Zong, H. Feng, F. R. Yu, N. Zhao, T. Yang, and B. Hu, "Optimal Transceiver Design for SWIPT in K -User MIMO Interference Channels," IEEE Transactions on Wireless Communications, vol. 15, no. 1, pp. 430-445, Jan 2016.

[204] E. G. Larsson, O. Edfors, F. Tufvesson, and T. L. Marzetta, "Massive MIMO for next generation wireless systems," IEEE Communications Magazine, vol. 52, no. 2, pp. 186-195, February 2014.

[205] G. Yang, C. K. Ho, R. Zhang, and Y. L. Guan, "Throughput Optimization for Massive MIMO Systems Powered by Wireless Energy Transfer," IEEE Journal on Selected Areas in Communications, vol. 33, no. 8, pp. 1640-1650, Aug 2015.

[206] L. Zhao, X. Wang, and K. Zheng, "Downlink Hybrid Information and Energy Transfer With Massive MIMO," IEEE Transactions on Wireless Communications, vol. 15, no. 2, pp. 1309-1322, Feb 2016.

[207] L. Zhao, K. Zheng, H. Yang, and W. Xiang, "Beamformer Design and Utility Optimization for Hybrid Information and Energy Transfer With Massive MIMO," IEEE Systems Journal, vol. PP, no. 99, pp. 1-12, 2016.

[208] A. Boaventura, D. Belo, R. Fernandes, A. Collado, A. Georgiadis, and N. B. Carvalho, "Boosting the Efficiency: Unconventional Waveform Design for Efficient Wireless Power Transfer," IEEE Microwave Magazine, vol. 16, no. 3, pp. 87-96, April 2015.

[209] B. Clerckx, E. Bayguzina, D. Yates, and P. D. Mitcheson, "Waveform optimization for Wireless Power Transfer with nonlinear energy harvester modeling," in 2015 International Symposium on Wireless Communication Systems (ISWCS), Aug 2015, pp. 276-280.

[210] K. Yang, S. Martin, D. Quadri, J. Wu, and G. Feng, "Energy-efficient downlink resource allocation in heterogeneous ofdma networks," IEEE Transactions on Vehicular Technology, vol. 66, no. 6, pp. 5086-5098, June 2017.

[211] X. Zhou, R. Zhang, and C. K. Ho, "Wireless information and power transfer in multiuser OFDM systems," in 2013 IEEE Global Communications Conference (GLOBECOM), Dec 2013, pp. 4092-4097.

[212] S. Yin and Z. Qu, "Resource Allocation in Multiuser OFDM Systems With Wireless Information and Power Transfer," IEEE Communications Letters, vol. 20, no. 3, pp. 594-597, March 2016.

[213] R. Wang and D. R. Brown, "Throughput maximization in wireless powered communication networks with energy saving," in 2014 48th Asilomar Conference on Signals, Systems and Computers, Nov 2014, pp. 516-520.

[214] W. Wang, L. Li, Q. Sun, and J. Jin, "Power Allocation in Multiuser MIMO Systems for Simultaneous Wireless Information and Power Transfer," in 2013 IEEE 78th Vehicular Technology Conference (VTC Fall), Sept 2013, pp. 1-5.

[215] J. Rubio, A. Pascual-Iserte, D. P. Palomar, and A. Goldsmith, "Joint Optimization of Power and Data Transfer in Multiuser MIMO Systems," IEEE Transactions on Signal Processing, vol. 65, no. 1, pp. 212-227, Jan 2017.

[216] Q. Shi, W. Xu, J. Wu, E. Song, and Y. Wang, "Secure beamforming for mimo broadcasting with wireless information and power transfer," IEEE Transactions on Wireless Communications, vol. 14, no. 5, pp. 2841-2853, May 2015.
[217] L. Liu, R. Zhang, and K. C. Chua, "Multi-Antenna Wireless Powered Communication With Energy Beamforming," IEEE Transactions on Communications, vol. 62, no. 12, pp. 4349-4361, Dec 2014.

[218] H. Ju and R. Zhang, "User cooperation in wireless powered communication networks," in 2014 IEEE Global Communications Conference, Dec 2014, pp. 1430-1435.

[219] G. Yang, C. K. Ho, R. Zhang, and Y. L. Guan, "Throughput Optimization for Massive MIMO Systems Powered by Wireless Energy Transfer," IEEE Journal on Selected Areas in Communications, vol. 33, no. 8, pp. 1640-1650, Aug 2015.

[220] S. Zhang, H. Zhang, and L. Yang, "Max-min fair robust beamforming design for multi-user MISO SWIPT systems," in 2016 IEEE International Conference on Communication Systems (ICCS), Dec 2016, pp. $1-5$.

[221] Q. Shi, L. Liu, W. Xu, and R. Zhang, "Joint Transmit Beamforming and Receive Power Splitting for MISO SWIPT Systems," IEEE Transactions on Wireless Communications, vol. 13, no. 6, pp. 3269-3280, June 2014

[222] J. Liao, M. R. A. Khandaker, and K. K. Wong, "Robust Power-Splitting SWIPT Beamforming for Broadcast Channels," IEEE Communications Letters, vol. 20, no. 1, pp. 181-184, Jan 2016.

[223] F. Wang, T. Peng, Y. Huang, and X. Wang, "Robust Transceiver Optimization for Power-Splitting Based Downlink MISO SWIPT Systems," IEEE Signal Processing Letters, vol. 22, no. 9, pp. 1492-1496, Sept 2015.

[224] Z. Chu, Z. Zhu, W. Xiang, and J. Hussein, "Robust beamforming and power splitting design in MISO SWIPT downlink system," IET Communications, vol. 10, no. 6, pp. 691-698, 2016.

[225] S. Timotheou, G. Zheng, C. Masouros, and I. Krikidis, "Exploiting Constructive Interference for Simultaneous Wireless Information and Power Transfer in Multiuser Downlink Systems," IEEE Journal on Selected Areas in Communications, vol. 34, no. 5, pp. 1772-1784, May 2016.

[226] Y. Cai, M. M. Zhao, Q. Shi, B. Champagne, and M. J. Zhao, "Joint Transceiver Design Algorithms for Multiuser MISO Relay Systems With Energy Harvesting," IEEE Transactions on Communications, vol. 64, no. 10, pp. 4147-4164, Oct 2016.

[227] H. Zhang, C. Li, Y. Huang, and L. Yang, "Secure Beamforming for SWIPT in Multiuser MISO Broadcast Channel With Confidential Messages," IEEE Communications Letters, vol. 19, no. 8, pp. 13471350, Aug 2015

[228] O. T. Demir and T. E. Tuncer, "Antenna Selection and Hybrid Beamforming for Simultaneous Wireless Information and Power Transfer in Multi-Group Multicasting Systems," IEEE Transactions on Wireless Communications, vol. 15, no. 10, pp. 6948-6962, Oct 2016.

[229] B. Xu, Y. Zhu, and R. Zhang, "Optimized Power Allocation for Interference Channel With SWIPT," IEEE Wireless Communications Letters, vol. 5, no. 2, pp. 220-223, April 2016.

[230] M. M. Zhao, Y. Cai, Q. Shi, B. Champagne, and M. J. Zhao, "Robust Transceiver Design for MISO Interference Channel With Energy Harvesting," IEEE Transactions on Signal Processing, vol. 64, no. 17, pp. 4618-4633, Sept 2016.

[231] S. Timotheou, I. Krikidis, G. Zheng, and B. Ottersten, "Beamforming for MISO Interference Channels with QoS and RF Energy Transfer," IEEE Transactions on Wireless Communications, vol. 13, no. 5, pp. 2646-2658, May 2014.

[232] Z. Zhu, Z. Wang, Z. Chu, X. Gao, Y. Zhang, and J. Cui, "Robust beamforming based on transmit power analysis for multiuser multipleinput-single-output interference channels with energy harvesting," IET Communications, vol. 10, no. 10, pp. 1221-1228, 2016.

[233] S. Fu, H. Wen, J. Wu, and B. Wu, "Energy-efficient precoded coordinated multi-point transmission with pricing power game mechanism," IEEE Systems Journal, vol. 11, no. 2, pp. 578-587, June 2017.

[234] K. Liang, L. Zhao, K. Yang, G. Zheng, and W. Ding, "A Fair Power Splitting Algorithm for Simultaneous Wireless Information and Energy Transfer in CoMP Downlink Transmission," Wireless Personal Communications, vol. 85, no. 4, pp. 2687-2710, 2015.

[235] Z. Zong, H. Feng, F. R. Yu, N. Zhao, T. Yang, and B. Hu, "Optimal Transceiver Design for SWIPT in K -User MIMO Interference Channels," IEEE Transactions on Wireless Communications, vol. 15, no. 1, pp. 430-445, Jan 2016.

[236] D. W. K. Ng and R. Schober, "Resource allocation for coordinated multipoint networks with wireless information and power transfer," in 2014 IEEE Global Communications Conference, Dec 2014, pp. 42814287 . 
[237] — "Secure and Green SWIPT in Distributed Antenna Networks With Limited Backhaul Capacity," IEEE Transactions on Wireless Communications, vol. 14, no. 9, pp. 5082-5097, Sept 2015.

[238] K. Liang, L. Zhao, Z. Ding, and H. H. Chen, "Double Side Signal Splitting SWIPT for Downlink CoMP Transmissions With Capacity Limited Backhaul," IEEE Communications Letters, vol. 20, no. 12, pp. 2438-2441, Dec 2016.

[239] W. N. S. F. W. Ariffin, X. Zhang, and M. R. Nakhai, "Sparse beamforming for real-time energy trading in CoMP-SWIPT networks," in 2016 IEEE International Conference on Communications (ICC), May 2016, pp. 1-6.

[240] J. F. Benders, "Partitioning procedures for solving mixed-variables programming problems," Numerische Mathematik, vol. 4, no. 1, pp. 238-252, Dec 1962. [Online]. Available: https://doi.org/10.1007/ BF01386316

[241] P. Ramezani and M. R. Pakravan, "Overview of MAC protocols for energy harvesting wireless sensor networks," in 2015 IEEE 26th Annual International Symposium on Personal, Indoor, and Mobile Radio Communications (PIMRC), Aug 2015, pp. 2032-2037.

[242] F. Iannello, O. Simeone, and U. Spagnolini, "Medium Access Control Protocols for Wireless Sensor Networks with Energy Harvesting," IEEE Transactions on Communications, vol. 60, no. 5, pp. 1381-1389, May 2012.

[243] M. Y. Naderi, P. Nintanavongsa, and K. R. Chowdhury, "RF-MAC: A Medium Access Control Protocol for Re-Chargeable Sensor Networks Powered by Wireless Energy Harvesting," IEEE Transactions on Wireless Communications, vol. 13, no. 7, pp. 3926-3937, July 2014.

[244] F. Shahzad and T. R. Sheltami, "An efficient MAC scheme in wireless sensor network with energy harvesting (EHWSN) for cloud based applications," in 2015 IEEE 40th Local Computer Networks Conference Workshops (LCN Workshops), Oct 2015, pp. 783-788.

[245] K. J. S., S. V. Rao, and S. S. Pillai, "Duty cycle adapted MAC for Wireless Sensor Networks with energy harvesting," in 2015 International Conference on Control Communication Computing India (ICCC), Nov 2015, pp. 685-690.

[246] T. D. Nguyen, J. Y. Khan, and D. T. Ngo, "An Adaptive MAC Protocol for RF Energy Harvesting Wireless Sensor Networks," in 2016 IEEE Global Communications Conference (GLOBECOM), Dec 2016, pp. 1-

[247] J. Kim and J.-W. Lee, "Energy adaptive MAC for wireless sensor networks with RF energy transfer: algorithm, analysis, and implementation," Telecommunication Systems, vol. 64, no. 2, pp. 293-307, 2017.

[248] P. Tamilarasi and B. Lavenya, "Energy and throughput enhancement in wireless powered communication networks using RF-MAC and CSMA," in 2015 International Conference on Innovations in Information, Embedded and Communication Systems (ICIIECS), March 2015, pp. $1-4$.

[249] A. M. Bedewy, A. A. El-Sherif, K. G. Seddik, and T. ElBatt, "Cooperative MAC for Cognitive Radio Network with Energy Harvesting and Randomized Service Policy," in 2015 IEEE Globecom Workshops (GC Wkshps), Dec 2015, pp. 1-7.

[250] M. Sami, N. K. Noordin, and M. Khabazian, "A TDMA-Based Cooperative MAC Protocol for Cognitive Networks With Opportunistic Energy Harvesting," IEEE Communications Letters, vol. 20, no. 4, pp. 808-811, April 2016.

[251] X. Qi, K. Wang, D. Yue, L. Shu, Y. Liu, and H. Zhao, "Adaptive TDMA-based MAC protocol in energy harvesting wireless body area network for mobile health," in IECON 2015 - 41st Annual Conference of the IEEE Industrial Electronics Society, Nov 2015, pp. 004175 004180.

[252] C. Ge, Z. Sun, N. Wang, K. Xu, and J. Wu, "Energy management in cross-domain content delivery networks: A theoretical perspective," IEEE Transactions on Network and Service Management, vol. 11, no. 3, pp. 264-277, September 2014.

[253] H. Huang, S. Guo, J. Wu, and J. Li, "Green Data Path for TCAMBased Software-Defined Networks," IEEE Communications Magazine, vol. 54, no. 11, pp. 194-201, November 2016.

[254] S. Fu, H. Wen, J. Wu, and B. Wu, "Cross-Networks Energy Efficiency Tradeoff: From Wired Networks to Wireless Networks," IEEE Access, vol. 5, pp. 15-26, June 2017.

[255] X. Bao and G. Ding, "An Routing Algorithm for Maximizing Network Performace in Energy Harvesting Wireless Sensor Network," in 2016 $3 r d$ International Conference on Information Science and Control Engineering (ICISCE), July 2016, pp. 1267-1270.

[256] M. E. Haque and U. Baroudi, "Energy Efficient Routing Scheme Using Leader Election in Ambient Energy Harvesting Wireless Ad Hoc and Sensor Networks," in 2015 IEEE SENSORS, Nov 2015, pp. 1-4.
[257] D. Liu, Y. Sun, Y. Yao, and C. Yin, "QoS-Aware Transmission Management for Energy Harvesting Wireless Sensor Networks," in 2016 23rd International Conference on Telecommunications (ICT), May 2016, pp. 1-7.

[258] J. Guo, T. Xu, T. Stavrinos, and M. Potkonjak, "Enabling Environmentally-Powered Indoor Sensor Networks with Dynamic Routing and Operation," in 2016 26th International Workshop on Power and Timing Modeling, Optimization and Simulation (PATMOS), Sept 2016, pp. 213-220.

[259] Q. Yao, A. Huang, H. Shan, T. Q. S. Quek, and W. Wang, "DelayAware Wireless Powered Communication Networks: Energy Balancing and Optimization," IEEE Transactions on Wireless Communications, vol. 15 , no. 8, pp. 5272-5286, Aug 2016.

[260] H. Darji and H. B. Shah, "Genetic Algorithm for Energy HarvestingWireless Sensor Networks," in 2016 IEEE International Conference on Recent Trends in Electronics, Information Communication Technology (RTEICT), May 2016, pp. 1398-1402.

[261] R. Mahajan and J. Kaur, "Appraisal of the Performance of Genetic Algorithm based WSN in Comparison with LEACH," International Journal of Latest Trends in Engineering and Technology, vol. 4, no. 3, September 2014.

[262] S. Feng, B. Qi, and L. Tang, "An Improved Energy-Efficient PEGASISBased Protocol in Wireless Sensor Networks," in 2011 Eights International Conference on Fuzzy Systems and Knowledge Discovery, July 2011.

[263] K. W. Chin, L. Wang, and S. Soh, "Joint Routing and Links Scheduling in Two-Tier Multi-Hop RF-Energy Harvesting Networks," IEEE Communications Letters, vol. 20, no. 9, pp. 1864-1867, Sept 2016.

[264] X. Zhang, Y. Ding, G. Yao, and K. Hao, "An Adaptive Clustering Routing Algorithm for Energy Harvesting-Wireless Sensor Networks," in 2016 IEEE Congress on Evolutionary Computation (CEC), July 2016, pp. 4699-4704.

[265] H. Li, C. Huang, P. Zhang, S. Cui, and J. Zhang, "Distributed Opportunistic Scheduling for Energy Harvesting Based Wireless Networks: A Two-Stage Probing Approach," IEEE/ACM Transactions on Networking, vol. 24, no. 3, pp. 1618-1631, June 2016.

[266] Y. Zhang, S. He, and J. Chen, "Data Gathering Optimization by Dynamic Sensing and Routing in Rechargeable Sensor Networks," IEEE/ACM Transactions on Networking, vol. 24, no. 3, pp. 1632-1646, June 2016

[267] A. Hentati, F. Abdelkefi, and W. Ajib, "Energy Allocation for Sensing and Transmission in WSNs with Energy Harvesting Tx/Rx," in 2015 IEEE 82nd Vehicular Technology Conference (VTC2015-Fall), Sept 2015, pp. 1-5.

[268] D. Zhang, Z. Chen, J. Ren, N. Zhang, M. K. Awad, H. Zhou, and X. S. Shen, "Energy-Harvesting-Aided Spectrum Sensing and Data Transmission in Heterogeneous Cognitive Radio Sensor Network," IEEE Transactions on Vehicular Technology, vol. 66, no. 1, pp. 831843, Jan 2017.

[269] K. Yang, S. Martin, C. Xing, J. Wu, and R. Fan, "Energy-Efficient Power Control for Device-to-Device Communications," IEEE Journal on Selected Areas in Communications, vol. 34, no. 12, pp. 3208-3220, December 2016.

[270] D. Munir, S. T. Shah, W. J. Lee, M. Y. Chung, and S. F. Hasan, "Selection of Relay UE with Energy Harvesting Capabilities in Public Safety Environment," in 2016 International Conference on Information Networking (ICOIN), Jan 2016, pp. 40-44.

[271] L. Jiang, H. Tian, Z. Xing, K. Wang, K. Zhang, S. Maharjan, S. Gjessing, and Y. Zhang, "Social-Aware Energy Harvesting Device-to-Device Communications in 5G Networks," IEEE Wireless Communications, vol. 23, no. 4, pp. 20-27, August 2016.

[272] R. Atat, L. Liu, H. Chen, J. Wu, H. Li, and Y. Yi, "Enabling CyberPhysical Communication in 5G Cellular Networks: Challenges, Spatial Spectrum Sensing, and Cyber-Security," IET Cyber-Physical Systems: Theory Applications, vol. 2, no. 1, pp. 49-54, April 2017.

[273] Y. Liu, L. Wang, S. A. R. Zaidi, M. Elkashlan, and T. Q. Duong, "Secure D2D Communication in Large-Scale Cognitive Cellular Networks: A Wireless Power Transfer Model," IEEE Transactions on Communications, vol. 64, no. 1, pp. 329-342, Jan 2016.

[274] U. Saleem, H. K. Qureshi, S. Jangsher, and M. Saleem, "Transmission Power Management for Throughput Maximization in Energy Harvesting Enabled D2D network," in 2016 IEEE Symposium on Computers and Communication (ISCC), June 2016, pp. 1078-1083.

[275] Y. Lu, W. Wang, L. Chen, Z. Zhang, and A. Huang, "Opportunistic Forwarding in Energy Harvesting Mobile Delay Tolerant Networks," in 2014 IEEE International Conference on Communications (ICC), June 2014, pp. 526-531. 
[276] O. Bouachir, A. B. Mnaouer, F. Touati, and D. Crescini, "Opportunistic Routing and Data Dissemination Protocol for Energy Harvesting Wireless Sensor Networks," in 2016 8th IFIP International Conference on New Technologies, Mobility and Security (NTMS), Nov 2016, pp. 1-5.

[277] H. Chen, Y. Li, J. L. Rebelatto, B. F. Uchôa-Filho, and B. Vucetic, "Harvest-Then-Cooperate: Wireless-Powered Cooperative Communications," IEEE Transactions on Signal Processing, vol. 63, no. 7, pp. 1700-1711, April 2015.

[278] P. V. Mekikis, A. Antonopoulos, E. Kartsakli, L. Alonso, and C. Verikoukis, "Connectivity Analysis in Wireless-Powered Sensor Networks with Battery-Less Devices," in 2016 IEEE Global Communications Conference (GLOBECOM), Dec 2016, pp. 1-6.

[279] K. Huang and V. K. N. Lau, "Enabling Wireless Power Transfer in Cellular Networks: Architecture, Modeling and Deployment," IEEE Transactions on Wireless Communications, vol. 13, no. 2, pp. 902 912, February 2014.

[280] S. Bi and R. Zhang, "Placement Optimization of Energy and Information Access Points in Wireless Powered Communication Networks," IEEE Transactions on Wireless Communications, vol. 15, no. 3, pp. 2351-2364, March 2016.

[281] Z. Zhang, Y. Li, K. Huang, and C. Liang, "Energy efficiency analysis of energy harvesting relay-aided cooperative networks," in 2015 13th International Symposium on Modeling and Optimization in Mobile, Ad Hoc, and Wireless Networks (WiOpt), May 2015, pp. 1-7.

[282] A. A. Nasir, D. T. Ngo, X. Zhou, R. A. Kennedy, and S. Durrani, "Joint Resource Optimization for Multicell Networks With Wireless Energy Harvesting Relays," IEEE Transactions on Vehicular Technology, vol. 65, no. 8, pp. 6168-6183, Aug 2016.

[283] B. Fan, S. Leng, K. Yang, and Q. Liu, "GPS: A method for data sharing in Mobile Social Networks," in 2014 IFIP Networking Conference, June 2014, pp. 1-9.

[284] B. Fan, S. Leng, C. Shao, Y. Zhang, and K. Yang, "Joint optimization of throwbox deployment and storage allocation in Mobile Social Networks," in 2015 IEEE International Conference on Communications (ICC), June 2015, pp. 1213-1218.

[285] B. Fan, S. Leng, K. Yang, and Q. Yu, "Socially-aware E-Box deployment schemes for joint data forwarding and energy harvesting," in 2016 IEEE International Conference on Communications (ICC), May 2016, pp. 1-6.

[286] A. Georgiadis, "Energy harvesting for autonomous wireless sensors and RFID's," in 2014 XXXIth URSI General Assembly and Scientific Symposium (URSI GASS), Aug 2014, pp. 1-5.

[287] K. Das, P. Zand, and P. Havinga, "Industrial Wireless Monitoring with Energy-Harvesting Devices," IEEE Internet Computing, vol. 21, no. 1, pp. 12-20, Jan 2017.

[288] V. A. Raj, "MEMS based Multi-Band Energy Harvesting for Wireless Sensor Network applications," in 2016 International Conference on Energy Efficient Technologies for Sustainability (ICEETS), April 2016, pp. $573-576$

[289] A. Berger, L. B. Hormann, C. Leitner, S. B. Oswald, P. Priller, and A. Springer, "Sustainable Energy Harvesting for Robust Wireless Sensor Networks in Industrial Applications," in 2015 IEEE Sensors Applications Symposium (SAS), April 2015, pp. 1-6.

[290] Q. Ibrahim, "Design, Implementation and Optimisation of an Energy Harvesting System for Vehicular Ad Hoc Networks' Road Side Units," IET Intelligent Transport Systems, vol. 8, no. 3, pp. 298-307, May 2014.

[291] R. Atallah, M. Khabbaz, and C. Assi, "Energy Harvesting in Vehicular Networks: a Contemporary Survey," IEEE Wireless Communications, vol. 23, no. 2, pp. 70-77, April 2016.

[292] F. Vazquez-Gallego, L. Alonso, and J. Alonso-Zarate, "Energy Harvesting-aware Contention Tree-Based Access for Wireless Machine-to-Machine Networks," in 2016 IEEE International Conference on Communications (ICC), May 2016, pp. 1-6.

[293] J. Rinne, J. Keskinen, P. R. Berger, D. Lupo, and M. Valkama, "Feasibility and Fundamental Limits of Energy-Harvesting based M2M Communications," in 2016 IEEE 27th Annual International Symposium on Personal, Indoor, and Mobile Radio Communications (PIMRC), Sept 2016, pp. 1-6.

[294] Y. Xiao, Z. Xiong, D. Niyato, Z. Han, and L. A. DaSilva, "Full-duplex Machine-to-Machine Communication for Wireless-Powered Internetof-Things," in 2016 IEEE International Conference on Communications (ICC), May 2016, pp. 1-6.

[295] D. Alanis, J. Hu, P. Botsinis, Z. Babar, S. X. Ng, and L. Hanzo, "Quantum-Assisted Joint Multi-Objective Routing and Load Balancing for Socially-Aware Networks," IEEE Access, vol. 4, pp. 9993-10 028, November 2016.
[296] G. Ding, Z. Tan, J. Wu, J. Zeng, and L. Zhang, "Indoor Fingerprinting Localization and Tracking System Using Particle Swarm Optimization and Kalman Filter," IEICE Transactions on Communications, vol. E98B, no. 3, pp. 502-514, March 2015.

[297] — , "Efficient Indoor Fingerprinting Localization Technique Using Regional Propagation Model," IEICE Transactions on Communications, vol. E97-B, no. 8, pp. 1728-1741, August 2014.

[298] J. Wu, S. Guo, J. Li, and D. Zeng, "Big Data Meet Green Challenges: Greening Big Data," IEEE Systems Journal, vol. 10, no. 3, pp. 873887, September 2016.

[299] _ -Big Data Meet Green Challenges: Big Data Toward Green Applications," IEEE Systems Journal, vol. 10, no. 3, pp. 888-900, September 2016.

[300] Q. Luo, W. Fang, J. Wu, and Q. Chen, "Reliable Broadband Wireless Communication for High Speed Trains Using Baseband Cloud," EURASIP Journal on Wireless Communications and Networking, vol. 2012, no. 1, pp. 285-296, September 2012.

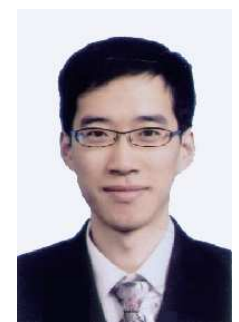

Jie Hu (S'11-M'16) received his B.Eng. and M.Sc. degrees from Beijing University of Posts and Telecommunications, China, in 2008 and 2011, respectively, and received the Ph.D. degree from the Faculty of Physical Sciences and Engineering, University of Southampton, U.K., in 2015. Since March 2016, he has been working with the School of Information and Communication Engineering, University of Electronic Science and Technology of China (UESTC), China, as an Associate Professor. His research now is funded by National Natural Science Foundation of China (NSFC). He is also in great partnership with industry, such as Huawei and ZTE. He has served for ZTE Communications as the guest editor of the special issue "Wireless Data and Energy Integrated Communication Networks". He also works as a TPC member in a number of prestigious IEEE conferences, such as IEEE ICC/Globecom/WSCP etc. He has a broad range of interests in wireless communication and networking, such as cognitive radio and cognitive networks, mobile social networks, simultaneous wireless information and power transfer as well as communication and computation convergence.

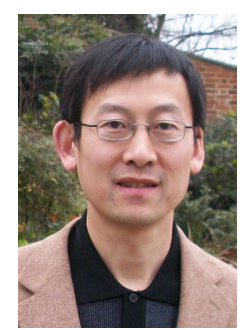

Kun Yang (SM'08) received his $\mathrm{PhD}$ from the Department of Electronic \& Electrical Engineering of University College London (UCL), UK, and MSc and BSc from the Computer Science Department of Jilin University, China. He is currently a Chair Professor in the School of Computer Science \& Electronic Engineering, University of Essex, leading the Network Convergence Laboratory (NCL), UK. He is also an affiliated professor at UESTC, China. Before joining in University of Essex at 2003, he worked at UCL on several European Union (EU) research projects for several years. His main research interests include wireless networks and communications, data and energy integrated networks, computation-communication cooperation. He manages research projects funded by various sources such as UK EPSRC, EU FP7/H2020 and industries. He has published $100+$ journal papers. He serves on the editorial boards of both IEEE and non-IEEE journals. He is a Senior Member of IEEE (since 2008) and a Fellow of IET (since 2009). 


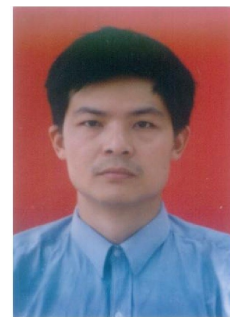

Guangjun Wen (M'04-SM'08) received the B.Sc degree and the M. Eng degree from Chongqing University at Chongqing, China, in 1986 and 1992, respectively, and $\mathrm{Ph} . \mathrm{D}$ degree University of Electronic Science and Technology of China(UESTC) at Chengdu, China, in 1998. From July 1986 to Feb. 1995, he was with Chongqing University,China, as a Lecturer. He was with UESTC,China, from July 1998 to May 2000, and then with Electronics and Telecommunication Research Institute,Korea, from May 2000 to May 2001, as a Postdoctoral Fellow. He was with Nanyang Technological University, Singapore, as a Research Fellow from May 2001 to Sept. 2002. He worked for VS Electronic Pte Ltd(Singapore) and Sumitomo Electric Group(Yokohama, Japan) as a Senior RF Design Engineer from Sept. 2002 to Aug. 2005. Since Jan. 2004, he is a Full Professor at UESTC,China. He was a visiting professor at University of California at Los Angeles (UCLA) from April 2015 to May 2015.

His research interests are in radio frequency integrated circuits and systems for various wireless communication systems, design of RFID tag and reader, circuit components and antennas design for the internet of things. He has authored or co-authored more than 200 journal papers and presented more than 130 conference papers. He hold more than 50 patents, and 2 books and 2 book chapter.

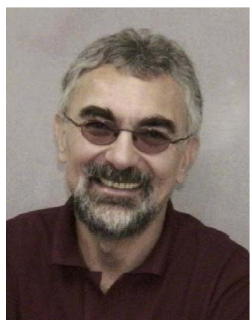

Lajos Hanzo ((M'91-SM'92-F'03)) FREng, FIEEE, FIET, Fellow of EURASIP, DSc received his degree in electronics in 1976 and his doctorate in 1983. In 2009 he was awarded an honorary doctorate by the Technical University of Budapest and in 2015 by the University of Edinburgh. In 2016 he was admitted to the Hungarian Academy of Science. During his 40-year career in telecommunications he has held various research and academic posts in Hungary, Germany and the UK. Since 1986 he has been with the School of Electronics and Computer Science, University of Southampton, UK, where he holds the chair in telecommunications. He has successfully supervised $112 \mathrm{PhD}$ students, co-authored 18 John Wiley/IEEE Press books on mobile radio communications totalling in excess of 10000 pages, published 1760 research contributions at IEEE Xplore, acted both as TPC and General Chair of IEEE conferences, presented keynote lectures and has been awarded a number of distinctions. Currently he is directing an academic research team, working on a range of research projects in the field of wireless multimedia communications sponsored by industry, the Engineering and Physical Sciences Research Council (EPSRC) UK, the European Research Council's Advanced Fellow Grant and the Royal Society's Wolfson Research Merit Award. He is an enthusiastic supporter of industrial and academic liaison and he offers a range of industrial courses. $\mathrm{He}$ is also a Governor of the IEEE ComSoc and VTS. During 2008- 2012 he was the Editor-in-Chief of the IEEE Press and a Chaired Professor also at Tsinghua University, Beijing. For further information on research in progress and associated publications please refer to http://www-mobile.ecs.soton.ac.uk 